\title{
An extended large sieve for Maaß cusp forms
}

\author{
Dissertation \\ zur Erlangung des mathematisch-naturwissenschaftlichen Doktorgrades \\ "Doctor rerum naturalium" \\ der Georg-August-Universität Göttingen \\ im Promotionsprogramm Mathematical Sciences \\ der Georg-August University School of Science (GAUSS)
}

vorgelegt von

\section{Christoph Renatus Ulrich Häußer}

aus Karlsruhe

Göttingen, 2018 
Betreuungsausschuss

Prof. Dr. Valentin Blomer

Mathematisches Institut, Georg-August-Universität Göttingen

Prof. Dr. Jörg Brüdern

Mathematisches Institut, Georg-August-Universität Göttingen

Mitglieder der Prüfungskommission

Referent: Prof. Dr. Valentin Blomer

Mathematisches Institut, Georg-August-Universität Göttingen

Korreferent: Prof. Dr. Jörg Brüdern

Mathematisches Institut, Georg-August-Universität Göttingen

Weitere Mitglieder der Prüfungskommission:

Prof. Dr. Dorothea Bahns

Mathematisches Institut, Georg-August-Universität Göttingen

Jun.-Prof. Dr. Christoph Lehrenfeld

Institut für Numerische und Angewandte Mathematik, Georg-August-Universität Göttingen

Prof. Dr. Russell Luke

Institut für Numerische und Angewandte Mathematik, Georg-August-Universität Göttingen

Prof. Dr. Chenchang Zhu

Mathematisches Institut, Georg-August-Universität Göttingen

Tag der mündlichen Prüfung: 29.08.2018 
To my parents 



\section{Acknowledgements}

While conducting my research I have been fortunate to enjoy the support of quite a few people, for which I would like to thank them here. First and foremost I am very grateful to Prof. Dr. Valentin Blomer, who has patiently encouraged my work with valuable advice and many inspiring discussions. My supervisor's guidance really was essential for me to write this thesis. I am thankful to Prof. Dr. Jörg Brüdern, my second thesis supervisor, for his counsel throughout my studies. For his guidance during my early stages as a mathematician I am grateful to Prof. Dr. Yiannis Petridis. Over the period of my time in Göttingen I have shared with my friends many happy moments and they have generously allowed me to lean on them for support. For this I would like to thank Alex, Marina, and Tomos. In particular I am indebted to Lisa, Max, and Vlad, for each one of them in their individual way have played an integral part in enabling me to complete this thesis. Finally, I would like to recognise the support of my parents. It is their consistent encouragement, advice, and affection, without which my efforts could not have proven successful. 



\section{Contents}

\begin{tabular}{llr}
\hline 1 & Preface & 8
\end{tabular}

\begin{tabular}{|ll|l}
2 & Introductory remarks & $\mathbf{1 6}$
\end{tabular}

2.1 Notational conventions . . . . . . . . . . . . . . . . . . . . . . 16

2.2 Maaß forms . . . . . . . . . . . . . . . . . . . . . . . . 17

2.3 The Bruggeman-Kuznetsov formula $\ldots \ldots \ldots \ldots \ldots$

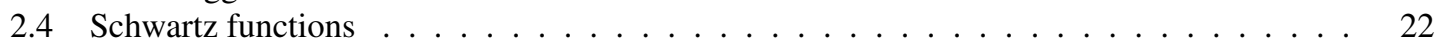

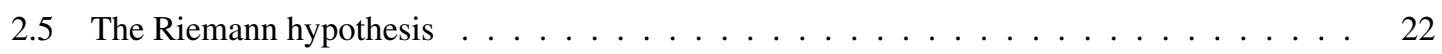

\begin{tabular}{|lll}
3 & An asymptotic expansion for the Bruggeman-Kuznetsov formula & 24
\end{tabular}

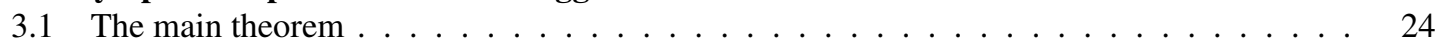

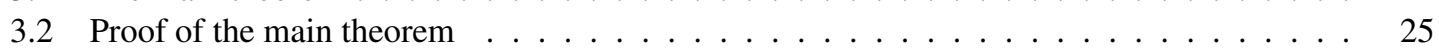

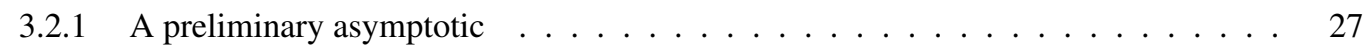

3.2 .2 Calculating the main terms . . . . . . . . . . . . . . . . . . . . . 29

3.3 Controlling an oscillatory integral $\ldots \ldots \ldots \ldots \ldots \ldots \ldots \ldots \ldots \ldots$

$\begin{array}{|lll|}4 & \text { An asymptotic for quadratic forms for the full spectrum } & 38\end{array}$

4.1 The Bruggeman-Kuznetsov formula for quadratic forms . . . . . . . . . . . . . . . 38

4.2 The main theorem $\ldots \ldots \ldots \ldots \ldots$

4.3 Proof of the main theorem . . . . . . . . . . . . . . . . . . . 40

4.4 Thinning out the main term . . . . . . . . . . . . . . . . . . . . . . . 42

4.5 The size of the error term . . . . . . . . . . . . . . . . . . . . . 45

4.6 Estimating the second main term $\mathscr{M}^{0}(\alpha) \ldots \ldots \ldots \ldots \ldots$. . . . . . . . . 47

4.6 .1 The size of $\mathscr{M}_{1}^{0}(\alpha) \ldots \ldots \ldots \ldots \ldots \ldots$

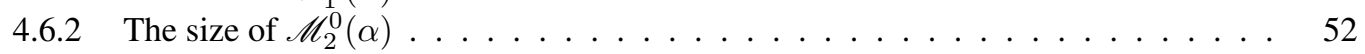

$4.7 \quad$ Large sieve inequalities for Bessel functions $1 \ldots \ldots \ldots \ldots \ldots \ldots$

$5 \quad$ An asymptotic formula for the discrete spectrum $\quad \mathbf{5 8}$

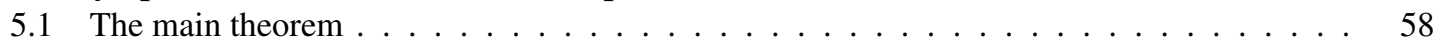

5.2 Matching Eisenstein terms $\ldots \ldots \ldots \ldots \ldots \ldots$

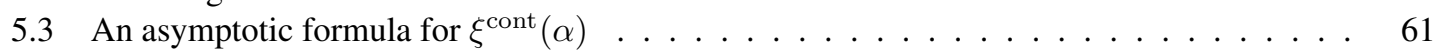

$5.3 .1 \quad$ Fourier coefficients of Eisenstein series $\ldots \ldots \ldots$. . . . . . . . . . 61

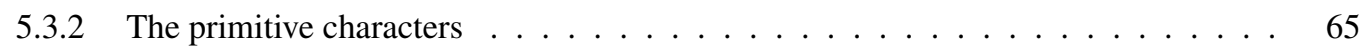

5.3 .3 The contribution of the principal character. . . . . . . . . . . . 69 
$6 \quad$ A large sieve for Maaß cusp forms

6.1 The main theorem . . . . . . . . . . . . . . . . . . . . . . . 72

6.2 Proof of the main theorem . . . . . . . . . . . . . . . . . . . . . . . 74

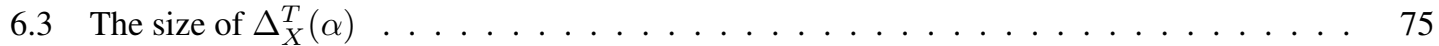

6.4 Large sieve inequalities for Bessel functions II . . . . . . . . . . . . . . . . . . . . . . . 79

\begin{tabular}{lll}
\hline & Appendix & 88
\end{tabular}

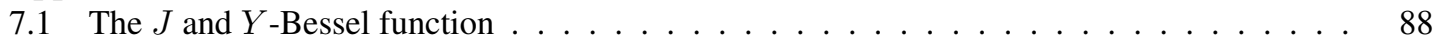

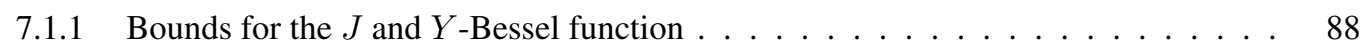

7.1 .2 The integral transform $\mathscr{J}$ of the $J$-Bessel function . . . . . . . . . . . . . 90

7.1.3 Isolating the oscillation of the $J$ and $Y$-Bessel function . . . . . . . . . . . 93

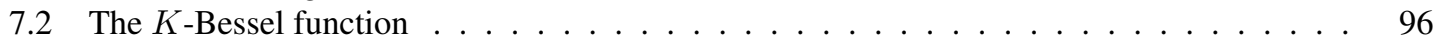

7.2 .1 The inverse Mellin transform of the $K$-Bessel function $\ldots \ldots \ldots$. . . . . . . . 96

7.2 .2 The Whittaker and the $K$-Bessel function $\ldots \ldots \ldots$. . . . . . . . . 97 


\section{Chapter 1}

\section{Preface}

Estimating the size of objects of interest is one of many key themes in analytic number theory. A particular type of inequalities is summarised under the label of "large sieves", which provides upper bounds for certain sums. Indeed in the context of large sieve inequalities we aim to bound for a finite collection of functions $u \in \mathcal{U}$, usually referred to as the harmonics, the sum

$$
\sum_{u \in \mathcal{U}}\left|\sum_{M<m \leq 2 M} a_{m} \theta_{u}(m)\right|^{2},
$$

where to every harmonic $u$ we attach a complex vector $\Theta(u)=\left(\theta_{u}(m)\right)_{m \in \mathbb{N}}$. As we range over the whole of $\mathcal{U}$, we assume that the $\mathrm{m}^{\text {th }}$ entries of the vectors $\Theta(u)$ may be estimated from above on average by a constant. In the above sum the entries of $\Theta(u)$ are summed against the corresponding entries of an arbitrary vector $\alpha$ of complex numbers, that has support in the interval $(M, 2 M]$, for an $M \geq 1$. For the latter we are going to denote the set of all such vectors by

$$
\mathbb{C}_{M}^{\infty}:=\left\{\alpha=\left(a_{m}\right)_{m \in \mathbb{N}} \in \mathbb{C}^{\infty} \mid \operatorname{supp}(\alpha) \subseteq(M, 2 M]\right\} .
$$

When we arrive at a bound for the sum in (1.1), we want to be able to judge the quality of the estimate obtained. In some sense a large upper bound is obtained by an application of the Cauchy Schwarz inequality, which yields

$$
\sum_{u \in \mathcal{U}}\left|\sum_{M<m \leq 2 M} a_{m} \theta_{u}(m)\right|^{2} \ll\|\alpha\|_{\infty}^{2} M^{2} \# \mathcal{U},
$$

with the vector $\alpha$ being estimated by the infinity norm ${ }^{(\mathrm{I})}$ defined by

$$
\|\alpha\|_{\infty}:=\max _{M<m \leq 2 M}\left|a_{m}\right| \text {. }
$$

However in general we hope to do much better than the Cauchy Schwarz bound. After opening the square in (1.1) we may identify the diagonal terms of the resulting $m, n$-summations, for which we cannot expect any cancellation, to see that a sum of this type does not exceed

$$
\|\alpha\|_{\infty}^{2} M \# \mathcal{U}+\text { off-diagonal terms. }
$$

\footnotetext{
${ }^{(\mathrm{I})}$ The following considerations may also be undertaken by using the 2 -norm, given by $\|\alpha\|:=\left(\sum_{m}\left|a_{m}\right|^{2}\right)^{1 / 2}$, to bound the vector $\alpha$. However for consistency with the result derived in this thesis, we choose to work with the infinity norm instead.
} 
Then for the off-diagonal terms in the latter we hope for square root cancellation in the $m, n$-summation, so that the diagonal terms in 1.3 dominate. Furthermore when we investigate the sum in 1.1 we desire a possible bound to be valid for every vector $\alpha \in \mathbb{C}_{M}^{\infty}$, in particular it therefore should hold for the specific choice

$$
\alpha\left(u^{*}\right)=\left(\overline{\theta_{u^{*}}(m)}\right)_{M<m \leq 2 M}
$$

as well, where we single out one element $u^{*}$ from the set of harmonics $\mathcal{U}$. Then, facilitated by the Cauchy Schwarz inequality, we easily find that an upper bound for the sum in (1.1) should be at least

$$
\|\alpha\|_{\infty}^{2} M^{2}+\text { additional terms, }
$$

for every $\alpha \in \mathbb{C}_{M}^{\infty}$ and with the "additional terms" here being dominated by 1.3 . Hence combining our considerations and assuming square root cancellation in the off-diagonal terms in $(1.3)$, we expect an estimate

$$
\sum_{u \in \mathcal{U}}\left|\sum_{M<m \leq 2 M} a_{m} \theta_{u}(m)\right|^{2} \ll(\# \mathcal{U}+M)\|\alpha\|_{\infty}^{2} M .
$$

This bound is clearly much more desirable that the Cauchy Schwarz bound introduced in (1.2), which, excluding trivial scenarios, will always dominate the bound (1.4). In conclusion in the context of large sieves we always hope to achieve an upper bound of the latter type and indeed in many instances an estimate of comparable quality as in (1.4) may be established.

As we will frequently employ it, we state an example in which the harmonics are a set of exponentials $e(*):=\exp (2 \pi \mathbf{i} *)$. Since those are periodic on $\mathbb{Z}$, it is clear that it suffices to split up the unit interval $\mathbb{R} / \mathbb{Z}$ in order to characterise the family of harmonics in this case. We do this by using Farey fractions with a denominator not exceeding $Y \geq 1$. Then we see easily that there are at most $\ll Y^{2}$ harmonics and from [14. Theorem 7.11] we know that in the context of our setup we have

$$
\sum_{y \leq Y} \sum_{h \bmod y}^{*}\left|\sum_{M<m \leq 2 M} a_{m} e\left(\frac{h m}{y}\right)\right|^{2} \ll\left(Y^{2}+M\right)\|\alpha\|_{\infty}^{2} M,
$$

for every vector $\alpha \in \mathbb{C}_{M}^{\infty}$. The latter large sieve inequality for the described particular family of exponentials, which we will in the following sometimes interpret as additive characters on the integers, is of the quality we hoped for in (1.4). There are many more examples for large sieves in many different contexts, which we will not focus on here and refer to [14, Chapter 7, p.169-195] for a more extended overview.

We will now discuss large sieves in relation to modular forms and state results for holomorphic- and for Maaß cusp forms. In both scenarios we begin by considering an orthonormal basis for the spaces of such function for a Dirichlet character $\chi$ of modulus $N$ for the Hecke congruence subgroup

$$
\Gamma_{0}(N):=\left\{\left(\begin{array}{ll}
a & b \\
c & d
\end{array}\right) \quad \mid\left(\begin{array}{ll}
a & b \\
c & d
\end{array}\right) \in S L_{2}(\mathbb{Z}) \& c \equiv 0(\bmod N)\right\}
$$

of $S L_{2}(\mathbb{Z})$ of level $N$. After that we are going to enlarge the number of harmonics by averaging over certain Dirichlet characters as well. To each such function we associate the vector having as entries the corresponding Fourier coefficient from the Fourier expansion of the particular harmonic (II)

\footnotetext{
${ }^{\text {(II) }}$ A Fourier expansion for those functions does always exist, since it is easy to show that they are periodic, as functions on the upper half plane $\mathbb{H}:=\{z=x+\mathrm{i} y \mid x+\mathrm{i} y \in \mathbb{C} \& y>0\}$, and the group $\Gamma_{0}(N)$ has cusps, with an expansion at each inequivalent one.
} 
We begin by considering holomorphic modular forms. The space $S_{k}\left(\Gamma_{0}(N) \backslash \mathbb{H} ; \chi\right)$ contains the holomorphic cusp forms of weight $k>2$ for the Hecke congruence subgroup of level $N$, and for the Dirichlet character $\chi$ of modulus $N$. Since these are periodic functions, at the cusp $\mathfrak{a} \sim \infty$ we can express them in terms of the Fourier expansion

$$
u(z)=\sum_{m \in \mathbb{N}} \psi_{u}(m) e(m z)
$$

where we rescale the arising coefficients via

$$
\psi_{u}^{*}(m):=\psi_{u}(m)\left(\frac{\Gamma(k-1) N}{(4 \pi m)^{k-1}}\right)^{1 / 2} .
$$

These renormalised Fourier coefficients are assumed to be bounded by a constant on average over the whole family. For the space $S_{k}\left(\Gamma_{0}(N) \backslash \mathbb{H} ; \chi\right)$ we will consider any orthonormal basis and denote it by $\mathcal{U}_{\chi}^{\text {hol }}$, which is going to be the set of harmonics in this context. Now to each member of this basis we associate a vector having the normalised Fourier coefficient $\psi_{u}^{*}(m)$ as the $\mathrm{m}^{\text {th }}$ entry and sum them against the corresponding places of any chosen $\alpha \in \mathbb{C}_{M}^{\infty}$ in the style of $\left[1.1\right.$. Then for instance for the trivial character $\chi \equiv 1^{(\text {III) }}$ we know from [14, Theorem 7.26] that

$$
\sum_{u \in \mathcal{U}_{\chi \equiv 1}^{\text {hol }}}\left|\sum_{M<m \leq 2 M} a_{m} \psi_{u}^{*}(m)\right|^{2} \ll(N+M)\|\alpha\|_{\infty}^{2} M
$$

where the bound holds for any $\alpha \in \mathbb{C}_{M}^{\infty}$. Now we would like to know how many elements the set of functions in question roughly contains. Indeed for a basis of holomorphic cusp forms for a Dirichlet character $\chi$ of modulus $N$ one may establish for instance from [5, Théorème 1, p.71], that for large enough weight $k$ we have

$$
\# \mathcal{U}_{\chi}^{\text {hol }} \asymp N k \prod_{p \mid N}\left(1+\frac{1}{p}\right)
$$

which we interpret in the way that we average over about $N$ different harmonics. With this in mind we observe that indeed the bound in (1.5) is of the desired quality as in (1.4). As described we will now turn our attention to an enlarged set of harmonics. To do this we additionally average the left had side of (1.5) over the Dirichlet characters of modulus $N$, that satisfy $\chi(-1)=(-1)^{k}$, in order to avoid spaces only containing the zero function. As a result we may now think about a large sieve inequality with respect to the set of harmonics being

$$
\mathcal{U}^{\text {hol }}:=\bigcup_{\substack{\chi \bmod N \\ \chi(-1)=(-1)^{k}}} \mathcal{U}_{\chi}^{\text {hol }}
$$

which is much larger than the previously considered single $\mathcal{U}_{\chi}^{\text {hol }}$, since summing 1.6 over the relevant characters shows that we should expect about $N^{2}$ functions in $\mathcal{U}^{\text {hol }}$. Indeed in [15] Iwaniec and Li establish a large sieve for precisely this family. Adapting their result [15, Corollary 12.1] to our notation, they show with the latter for prime level $N$ with $M \geq N$ that

$$
\sum_{u \in \mathcal{U}^{\text {hol }}}\left|\sum_{M<m \leq 2 M} a_{m} \psi_{u}^{*}(m)\right|^{2} \ll\left(\left(N^{3} M\right)^{1 / 2}+M\right)\|\alpha\|_{\infty}^{2} M^{1+\varepsilon},
$$

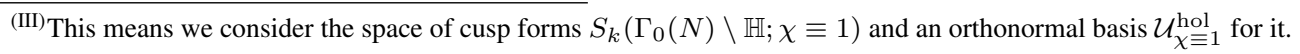


for every $\alpha \in \mathbb{C}_{M}^{\infty}$. This result is in a way unexpected. When we increased the size of the set of harmonics, we average over more objects, which we expect to behave independent of each other. Stronger cancellation effects should be the consequence. However the upper bound in 1.7 does not fall in line with this prediction. In fact it fails to achieve the quality we hope for in the context of large sieves as stated in (1.4), as we would expect a factor $\left(N^{2}+M\right)$ in the estimate rather than the always larger $\left(\left(N^{3} M\right)^{1 / 2}+M\right)^{(\mathrm{IV})}$.

In a next step we are now going to compare the holomorphic case with the scenario of Maaß cusp forms. To this end we start again by looking at the space of such forms for the Hecke congruence subgroup of level $N$ and for the Dirichlet character $\chi$ of modulus $N$, which we denote by $\mathcal{C}_{1 / 2+\mathrm{i} t}\left(\Gamma_{0}(N) \backslash \mathbb{H} ; \chi\right)$. Any function $u$ in the latter space is an eigenfunction of the hyperbolic Laplace operator

$$
\Delta_{\text {Laplace }}:=-y^{2}\left(\frac{\partial^{2}}{\partial x^{2}}+\frac{\partial^{2}}{\partial y^{2}}\right)
$$

with Laplace eigenvalue $\lambda_{u}=1 / 4+t_{u}^{2}$, for some $t_{u} \in(-\mathrm{i} / 2, \mathrm{i} / 2) \cup \mathbb{R}$. An orthonormal basis of the space $\mathcal{C}\left(\Gamma_{0}(N) \backslash \mathbb{H} ; \chi\right)$ is described by $\mathcal{U}_{\chi}^{\text {Maaß }}$. Maaß cusp forms are periodic functions and at the cusp $\mathfrak{a} \sim \infty$ therefore admit the Fourier expansion

$$
u(z)=y^{1 / 2} \sum_{m \in \mathbb{Z} \backslash\{0\}} \rho_{u}(m) K_{\mathrm{i} t_{u}}(2 \pi|m| y) e(m x),
$$

where we renormalise the Fourier coefficients by defining

$$
\rho_{u}^{*}(m):=\rho_{u}(m)\left(\frac{2 N}{\cosh \left(\pi t_{u}\right)}\right)^{1 / 2}
$$

for which we believe that they are on average bounded above by a constant. We define the set of Maaß cusp forms with a spectral parameter $t \in[0, T]$ by

$$
\mathcal{U}_{T}^{\mathrm{Maa}}:=\left\{u \in \mathcal{U}_{\chi}^{\mathrm{Maa}} \mid t \in[0, T] \& \chi \equiv 1\right\} .
$$

Then by Weyl's law we expect roughly $\# \mathcal{U}_{T}^{\mathrm{Maa} ß} \approx N T^{2}$ (cp. [13, (11.5), p.176] or [14, p.391]). Now, again adapting the result to our setup, we have from [14, Theorem 7.24] the inequality

$$
\sum_{u \in \mathcal{U}_{T}^{\text {Maaß }}}\left|\sum_{M<m \leq 2 M} a_{m} \rho_{u}^{*}(m)\right|^{2} \ll\left(N T^{2}+M \log M\right)\|\alpha\|_{\infty}^{2} M,
$$

for all $\alpha \in \mathbb{C}_{M}^{\infty}(\mathrm{V})$ With the comments made earlier about the rough size of $\mathcal{U}_{T}^{\mathrm{Maa}}{ }^{\mathrm{(}}$ this large sieve provides an upper bound again essentially of the optimal quality as in the spirit of (1.4). The latter is only missed by a factor of $\log M$, which we consider to be not significant in this context. As before in the case of holomorphic modular forms we now want to investigate the situation that arises provided we consider a larger family of Maaß cusp forms. To this end we deal with the set

$$
\mathcal{U}^{\mathrm{Maa} \beta}:=\bigcup_{\substack{\chi \bmod N \\ \chi(-1)=1}} \mathcal{U}_{\chi}^{\mathrm{Maa}}
$$

\footnotetext{
(IV) In the setup using the 2-norm to estimate the vector $\alpha$, Iwaniec and Li identify in [15] also vectors, for which they show that this unusually large sieve bound cannot be improved (cp. [15. Corollary 13.1]).

(V) In [6] Deshouillers and Iwaniec show a large sieve inequality very much in the same fashion as in 1.9]. They estimate essentially the same sum as on the left hand side in 1.9 with an upper bound, that, for $N$ prime and translated into the notation chosen in this thesis, reads $\left(N T^{2}+M^{1+\varepsilon}\right)\|\alpha\|_{\infty}^{2} M$ (cp. [6] Theorem 2]).
} 
where we sum of such Dirichlet characters of modulus $N$, for which the corresponding spaces do not solely contain the zero function. The subject of this thesis is to establish a large sieve inequality for the family of harmonics being $\mathcal{U}^{\text {Maaß }}$, in some sense weighted with some function $h$, to be specified in due course. Indeed we will provide a full proof for the following result.

Theorem 1.1. Suppose the Riemann hypothesis (in the form: The Riemann hypothesis 2.7; see Section 2.5) is true. Let $h$ be a Schwartz function satisfying the assumptions made in Section 2.4. We assume the Hecke congruence subgroup has odd prime level $N$. Then for every vector $\alpha \in \mathbb{C}_{M}^{\infty}$, with $M>N$, we have

$$
\sum_{u \in \mathcal{U}^{\mathrm{Maa} B}}\left|\sum_{M<m \leq 2 M} a_{m} \rho_{u}^{*}(m)\right|^{2} h\left(t_{u}\right) \ll\left(\left(N^{3} M\right)^{1 / 2}+M\right)\|\alpha\|_{\infty}^{2} M^{1+\varepsilon},
$$

for any small $\varepsilon>0$. The implied constant depends on the Schwartz function $h$.

As before we want to discuss the quality of the obtained upper bound. To be able to do this we need to have an approximate understanding of the number of harmonics weighted by the function $h$, over which we average. In fact again facilitated by Weyl's law (again cp. [13, (11.5), p.176] or [14, p.391]) we infer for $N$ a prime that

$$
\sum_{u \in \mathcal{U}^{\mathrm{Maa} B}} h\left(t_{u}\right) \approx N^{2} \int_{0}^{\infty} x h(x) d x
$$

where the involved integral converges, since we assume decay properties for the function $h$ as specified in Section 2.4 As the implied constant in the inequality in $(1.10)$ is allowed to be a function of the Schwartz function $h$, the integral in 1.11 may be absorbed thereof and we would hope for the factor $\left(N^{2}+M\right)$. Instead we establish the larger $\left(\left(N^{3} M\right)^{1 / 2}+M\right)$, since we operate under the assumption that $M>N$; in particular if $M$ and the level $N$ of the group are not of the same size, the bound we find might significantly exceed the expectation from the general setup of the large sieve, as explained earlier. This somewhat unexpected phenomenon resembles similar observations, as made in the holomorphic scenario by Iwaniec and $\mathrm{Li}$ in [15] and indeed a related speculation as therein (cp. [15. 1. Introduction; p. 543]) may hint towards an explanation for it. The inner sum on the left hand side in (1.10), which is

$$
\sum_{M<m \leq 2 M} a_{m} \rho_{u}^{*}(m)
$$

can be interpreted as the inner product of the two vectors $\alpha$ and $\Theta^{\mathrm{Maa} \beta}(u)=\left(\rho_{u}^{*}(m)\right)$ associated to each member $u \in \mathcal{U}^{\text {Maaß }}$ of the family of harmonics that we consider. The inner product measures in some sense the extent to which these two vectors are orthogonal and parallel respectively to each other. Precisely the closer they are to being parallel the larger the product is, with a maximum when they are perfectly parallel, and on the other hand the closer they are to being orthogonal the smaller the product is, with it attaining the value zero for them being perfectly orthogonal. If the vectors $\left\{\Theta^{\operatorname{Maa} \beta}(u) \mid u \in \mathcal{U}^{\operatorname{Maa} \beta}\right\}$ were to point into different directions arbitrarily, no matter how an $\alpha$ is chosen, the absolute value of the inner product 1.12) can only be large for a small number of vectors from this family, as $\alpha$ can only resemble the direction of a few of them at the same time. For most of the vectors in $\left\{\Theta^{\text {Maaß }}(u) \mid u \in \mathcal{U}^{\text {Maaß }}\right\}$ the vector $\alpha$ should then be to some extent orthogonal to them, resulting in each case in a smaller absolute contribution from the inner product $(1.12)$. Such a random distribution of the directions in which the vectors from the family $\left\{\Theta^{\mathrm{Maa} \beta}(u) \mid u \in \mathcal{U}^{\mathrm{Maa} \beta}\right\}$ point, would therefore make it plausible that on average over $\mathcal{U}^{\mathrm{Maa} \beta}$ the total contribution from all the possible inner products, that is essentially what we aim to bound on the left hand side in (1.10), should not be too large. Hence it may be reasonable to hope for an upper 
bound in the inequality in Theorem 1.1 of the same quality as the theoretical setup of the large sieve predicts.

However, the larger bound we obtain in reality in Theorem 1.1 suggests that we in fact encounter a slightly different situation. The inequality stated therein is supposed to hold for any choice of $\alpha \in \mathbb{C}_{M}^{\infty}$. In particular if the vectors $\left\{\Theta^{\mathrm{Maa}}(u) \mid u \in \mathcal{U}^{\mathrm{Maa}}\right\}$ were to favour a common direction the bound in 1.10 has to hold for vectors $\alpha$ aligning with it as well. This would result in the absolute value of the inner product (1.12) for such an $\alpha$ to be relatively large for many $u \in \mathcal{U}^{\mathrm{Maa} \beta}$ at the same time, which has to be accounted for in the estimate in (1.10), potentially resulting in it being larger than the expectation. Since this is in fact the case here, the inequality in Theorem 1.1 seems to indicate that the vectors $\left\{\Theta^{\operatorname{Maa}}(u) \mid u \in \mathcal{U}^{\text {Maaß}}\right\}$ do not point in different directions completely randomly, but are rather slightly oriented toward a mutual one.

Remark 1.2 (Structure of the proof). The argument we present in this thesis in support of Theorem 1.1 follows largely the structure and layout of the proof given by Iwaniec and Li in [15] to establish their equivalent results in the context of holomorphic modular forms (cp. (1.7) and [15] Corollary 12.1]). A number of the strategies and methods employed in [15] can be adapted to our situation of Maaß cusp forms. However a few aspects are also quite different and require additional attention.

Instead of the Petersson formula (cp. [14] Proposition 14.5]) in the holomorphic case the starting point, upon which the whole proof of Theorem 1.1 is built on, is the Bruggeman-Kuznetsov formula (see Section 2.3). In Chapter 3 the arithmetic side of the Bruggeman-Kuznetsov formula is developed into an asymptotic formula. After an application of Poisson summation the resulting integral is translated via Lemma 7.6. depending on the values of one parameter involved, into integrals over various Bessel functions and the Schwartz function $h$ (cp. Section 2.4) arising from the use of the Bruggeman-Kuznetsov formula. For the case of this particular parameter being equal to zero, Lemma 7.6 provides an integral of particular interest, those role we are going to interpret in due course. The transformation of terms facilitated by Lemma 7.6 is a key step in order to be able to obtain estimates for the corresponding terms of the desired quality. Although the same approach is used in [15], the resulting terms after the transformation are really quite different in our case. Not only do the terms corresponding to the holomorphic case look different, but also there is a whole range of nontrivial terms in the Maaß case that vanishes, once we operate in the context of holomorphic modular cusp forms. These additional terms are consequently featured in the results of the remaining chapters, derived from the material in Chapter 3 and require some careful treatment.

The Bruggeman-Kuznetsov formula equates to the arithmetic side a combination of two groups of terms on the spectral side. Those are terms containing the Fourier coefficients of Maaß cusp forms summed together with terms featuring the Fourier coefficients of Eisenstein series. These two parts of the spectral side are reminiscent respectively of the discrete and the continuous spectrum, resulting from spectral decomposition of the space $L^{2}\left(\Gamma_{0}(N) \backslash \mathbb{H} ; \chi\right)$ (cp. for primitive characters with the combination of [7. Proposition 4.1]) and [7 Proposition 4.2]) and with [14] Theorem 15.5] in case of the principal character $\chi_{0}$ ) with respect to the hyperbolic Laplace operator $\Delta_{\text {Laplace, }}$ being a key ingredient in establishing the Bruggeman-Kuznetsov formula. The terms coming from the discrete spectrum are ultimately the object of our investigations. However the part being the representative of the continuous spectrum slightly gets in the way. It has a corresponding term on the arithmetic side of the formula, which is the one featuring the integral given by Lemma 7.6 for one of the parameters being equal to zero. Both terms on either side of the BruggemanKuznetsov formula can be shown to coincide up to an error term of acceptable magnitude for our purposes. Establishing this link between the two terms is essentially subject of Chapter 5 and has no correspondence whatsoever with the procedure used in the holomorphic case in [15], since the Petersson formula (cp. [14] Proposition 14.5]) being the staring point in the latter scenario does not feature any terms resembling the ones containing Fourier coefficients of Eisenstein series as the Bruggeman-Kuznetsov formula does.

Finally in Chapter 6 we establish an asymptotic formula for terms containing the Fourier coefficients of 
Maaß cusp forms. The particular asymptotic expansion we derive therein has its correspondence in the holomorphic case in [15. Theorem 1.1]. Both results share the same feature that the number of terms in the main term may be increased or decreases according to the choice of some parameter, causing respectively a smaller/larger error term. Following the same strategy as in [15], from the asymptotic expansion we show in Chapter 6, we immediately conclude the large sieve inequality stated in Theorem 1.1, the main result of this thesis.

Remark 1.3 (The Riemann hypothesis). In the proof of Theorem 1.1 we rely at a certain point on the validity of the Riemann hypothesis $(\mathrm{RH})$ in the form we are going to specify in two equivalent formulations in Section 2.5. As this famous conjecture is unproven, our result is therefore conditional thereof. However since it is widely assumed for the statement to hold up eventually, we go forward employing it without proof, as it has become common practice in the field. It is probably worth pointing out that even in the absence of the Riemann hypothesis most of the arguments presented in this thesis remain true. It is only in Chapter 5 that we give arguments that employ RH. Since this chapter is an integral part in the process of deriving our main result, Theorem 1.1, all subsequent conclusions relying on this part of the thesis thus depend on the conjecture. 



\section{Chapter 2}

\section{Introductory remarks}

This chapter does not contain any original work of us. Its purpose is merely to introduce some notation, conventions, and obvious observations, which we will use frequently throughout this thesis and to collect some standard facts about certain objects from the literature. Furthermore we are also going to introduce the Bruggeman-Kuznetsov formula (cp. Section 2.3. A result of this type was first established by Kuznetsov and independently by Bruggeman, the mathematicians the formula is named after. This formula is a standard result from the literature, on which our considerations in subsequent chapters entirely hinge on. We conclude this chapter with some remarks about the famous and so far unproven Riemann hypothesis.

\subsection{Notational conventions}

In this thesis we will follow in general the standard notational conventions common in analytic number theory. However in this section we will highlight few points of particular importance in our context. A sum over residue classes with an asterisk as superscript, i.e. for instance

$$
\sum_{x \bmod t}^{*}
$$

denotes the summation over such residue classes, which are invertible with respect to the indicated modulus. When we sum Dirichlet characters of modulus $N$, by writing

$$
\sum_{\chi \bmod N}^{+}
$$

with a plus as superscript we only consider those that satisfy $\chi(-1)=1$. We write

$$
\mathbb{R}_{>0}:=\{x \in \mathbb{R} \mid x>0\} .
$$

Similar notation is meant to be understood with a definition in the same spirit in mind. Quite frequently we will make use of the symbols $\varepsilon, \delta$ or alterations thereof. These will, even if not explicitly indicated, denote arbitrarily small numbers in $\mathbb{R}_{>0}$. Occasionally we will also implicitly assume that such $\varepsilon$ or $\delta$ are in fact small enough for our purposes, in order to ensure the validity of the claimed calculations. Of particular importance is furthermore the fact that the actual value, which such $\varepsilon$ or $\delta$ represent might not be the same from one appearance to the next. In our estimates the implicit constants often depend on $\varepsilon$ or $\delta$, which we omit to point out when this is the case. The $\delta$ introduced above should not be confused however with the 
Kronecker delta, which is defined by

$$
\delta_{m, n}:= \begin{cases}1, & \text { if } m=n \\ 0, & \text { if } m \neq n\end{cases}
$$

unless explicitly specified otherwise in a particular context. In quite a few instances we are going to talk about the support of certain objects. If we consider a complex vector $\alpha=\left(a_{m}\right)_{m \in \mathbb{N}} \in \mathbb{C}^{\infty}$ we define its support by

$$
\operatorname{supp}(\alpha):=\left\{m \in \mathbb{N} \mid a_{m} \neq 0\right\} .
$$

On the other hand we understand the support of a function $f: \mathbb{R} \rightarrow \mathbb{R}$ to be

$$
\operatorname{supp}(f):=\overline{\{f(x) \in \mathbb{R} \mid f(x) \neq 0\}}
$$

For functions mapping different domains (other than $\mathbb{R}$ ) into some other space (again not necessarily $\mathbb{R}$ ), its support is given accordingly in a similar fashion.

\subsection{Maaß forms}

The object of this thesis is to establish Theorem 1.1. It provides an upper bound for sums involving the Fourier coefficients of a certain family of Maaß cusp forms. In order to put this into perspective we are going to give in this section a brief exposition of these functions. This is fairly standard in the literature and we follow to some extent the layout of the presentation chosen in [13, Chapter 3] and [14, Chapter 15] (cp. the latter two sources for additional details, extending beyond the scope of the exposition given here). Although the setup to follow could be in principle done for any Fuchsian group of the first kind, for the relevance to this thesis we restrict to considering the Hecke congruence subgroup of level $N$, given by

$$
\Gamma_{0}(N)=\left\{\left(\begin{array}{ll}
a & b \\
c & d
\end{array}\right) \mid\left(\begin{array}{ll}
a & b \\
c & d
\end{array}\right) \in S L_{2}(\mathbb{Z}) \& c \equiv 0(\bmod N)\right\},
$$

only. The group $S L_{2}(\mathbb{R})$, and therefore any subgroup thereof, acts on the upper half plane $\mathbb{H}=\{z=$ $x+\mathrm{i} y \mid x+\mathrm{i} y \in \mathbb{C} \& y>0\}$ by Möbius transformations, described by

$$
\gamma z:=\frac{a z+b}{c z+d}, \text { for any } \gamma=\left(\begin{array}{ll}
a & b \\
c & d
\end{array}\right) \in S L_{2}(\mathbb{R}) .
$$

A point $\mathfrak{a}$ on the boundary of $\mathbb{H}$, that satisfies $\gamma \mathfrak{a}=\mathfrak{a}$ for some parabolic element $\gamma \in \Gamma_{0}(N)$, is defined to be a cusp of the Hecke congruence subgroup, with the stabiliser of any such cusp being given by the cyclic group $\Gamma_{\mathfrak{a}}:=\left\{\gamma \in \Gamma_{0}(N) \mid \gamma \mathfrak{a}=\mathfrak{a}\right\}$. Two cusps are said to be equivalent, denoted by $\mathfrak{a} \sim \mathfrak{b}$, if for some $\gamma \in \Gamma_{0}(N)$ we have $\gamma \mathfrak{a}=\mathfrak{b}$, which gives rise to the matter of identifying the equivalence classes of cusps for this group. In order to do so we define the set $L(k)$, for every $k$ dividing the level $N$ of the group, in the following manner: First it needs to be a subset of $\mathbb{N} \cup\{0\}$, for which all of its elements $l$ satisfy $\operatorname{gcd}(l, k)=1$. Secondly this set has to precisely stand for the residue classes $\{0,1,2, \ldots, \operatorname{gcd}(k, N / k)-1\}$ of the modulus $\operatorname{gcd}(k, N / k)$. With this notation in place, the Hecke congruence subgroup of level $N$ possesses the set

$$
\{l / k|k| N \& l \in L(k)\}
$$

of representatives of inequivalent cusps, which means there are

$$
\sum_{k \mid N} \varphi((k, N / k))
$$


many equivalence classes of inequivalent cusps for $\Gamma_{0}(N)$, where $\varphi$ denotes the Euler function (for details about the cusps of $\Gamma_{0}(N)$ cp. for instance [2, 5. Proof of Lemma 1, p. 64] and also [6, Lemma 2.3]). Now in the context of the Hecke congruence subgroup we may define the space of automorphic functions to be

$$
\mathcal{A}\left(\Gamma_{0}(N) \backslash \mathbb{H} ; \chi\right):=\left\{\begin{array}{l|l}
u: \mathbb{H} \rightarrow \mathbb{C} & \begin{array}{c}
u(\gamma z)=\chi(\gamma) u(z), \text { for every } \gamma \in \Gamma_{0}(N) \\
\& u(z)=o(\exp (2 \pi \Im(z))), \text { as } \Im(z) \rightarrow \infty
\end{array}
\end{array}\right\}
$$

where we define for any Dirichlet character of modulus $N$ that $\chi(\gamma):=\chi(d)$, with $\gamma \in \Gamma_{0}(N)$ having entries as in 2.1). We highlight the fact that since $-\mathrm{I} \in \Gamma_{0}(N)$ the space $\mathcal{A}\left(\Gamma_{0}(N) \backslash \mathbb{H} ; \chi\right)$ only contains the zero function in case of $\chi(-1)=-1$. Hence for the remainder of this thesis we will exclude this trivial case and only consider Maaß forms satisfying a transformation behaviour with a character having the correct parity, i.e Dirichlet characters that admit $\chi(-1)=1$. We want to consider eigenfunctions of the hyperbolic Laplace operator

$$
\Delta_{\text {Laplace }}=-y^{2}\left(\frac{\partial^{2}}{\partial x^{2}}+\frac{\partial^{2}}{\partial y^{2}}\right)
$$

for with we define the space of (automorphic) Maaß forms given by

$$
\mathcal{A}_{s}\left(\Gamma_{0}(N) \backslash \mathbb{H} ; \chi\right):=\left\{u \in \mathcal{A}\left(\Gamma_{0}(N) \backslash \mathbb{H} ; \chi\right) \mid \Delta_{\text {Laplace }} u=s(1-s) u\right\},
$$

for some $s \in \mathbb{C}$. Then for instance from [13, Proposition 1.5] we know that any $u \in \mathcal{A}_{s}\left(\Gamma_{0}(N) \backslash \mathbb{H} ; \chi\right)$ we have the Fourier expansion

$$
u(z)=c_{1} y^{s}+c_{2}\left(y^{1-s}+\delta_{s, 1 / 2} \cdot y^{1 / 2} \log y\right)+y^{1 / 2} \sum_{m \in \mathbb{Z} \backslash\{0\}} \rho_{u}(m) K_{s-1 / 2}(2 \pi|m| y) e(m x),
$$

for $c_{1}, c_{2} \in \mathbb{C}$ and where $K$ denotes the $K$-Bessel function (cp. [13, (1.26)]). For automorphic functions, such that the following integral makes sense, we introduce the inner product

$$
\langle f, g\rangle:=\int_{\Gamma_{0}(N) \backslash \mathbb{H}} f(z) \overline{g(z)} d \mu(z),
$$

for the $S L_{2}(\mathbb{R})$, and therefore also $\Gamma_{0}(N)$, invariant measure $d \mu(z):=d x d y / y^{2}$. Based on the latter inner product we now define the Hilbert space

$$
L^{2}\left(\Gamma_{0}(N) \backslash \mathbb{H} ; \chi\right):=\left\{u \in \mathcal{A}\left(\Gamma_{0}(N) \backslash \mathbb{H} ; \chi\right) \mid\|u\|:=\langle u, u\rangle^{1 / 2}<\infty\right\} .
$$

Now if $\mathfrak{a}$ is any cusp of the Hecke congruence subgroup, we may find a scaling matrix $\sigma_{\mathfrak{a}} \in S L_{2}(\mathbb{R})$, defined as the matrix admitting $\sigma_{\mathfrak{a}} \infty=\mathfrak{a}$ as well as the conjugation $\sigma_{\mathfrak{a}}^{-1} \Gamma_{\mathfrak{a}} \sigma_{\mathfrak{a}}=\Gamma_{\infty}$ (I) From these definitions we observe immediately that for any $u \in \mathcal{A}_{s}\left(\Gamma_{0}(N) \backslash \mathbb{H} ; \chi\right)$ the function $u_{\mathfrak{a}}(z):=u\left(\sigma_{\mathfrak{a}} z\right)$ satisfies $u_{\mathfrak{a}}(z+h)=u_{\mathfrak{a}}(z)$, for any $h \in \mathbb{N}$, and therefore has a Fourier expansion

$$
u_{\mathfrak{a}}(z)=\sum_{m \in \mathbb{Z}} \rho_{u, m}(y ; \mathfrak{a}) e(m x) .
$$

Finally, we are in a position to give the definition of the objects we are mainly interested in. Indeed we denote the space of Maaß cusp forms with a Laplace eigenvalue $\lambda=s(1-s)$ by

$$
\mathcal{C}_{s}\left(\Gamma_{0}(N) \backslash \mathbb{H} ; \chi\right):=\left\{u \in L^{2}\left(\Gamma_{0}(N) \backslash \mathbb{H} ; \chi\right) \cap \mathcal{A}_{s}\left(\Gamma_{0}(N) \backslash \mathbb{H} ; \chi\right) \mid \rho_{u, 0}(y ; \mathfrak{a})=0 \text { for all cusps } \mathfrak{a}\right\}
$$

where $\rho_{u, 0}(y ; \mathfrak{a})$ are the zeroth coefficients in the Fourier expansion as in 2.3 for any cusp $\mathfrak{a}$ of $\Gamma_{0}(N)$. The space of all Maaß cusp forms in $L^{2}\left(\Gamma_{0}(N) \backslash \mathbb{H} ; \chi\right)$ is defined to be $\mathcal{C}\left(\Gamma_{0}(N) \backslash \mathbb{H} ; \chi\right)$, which is a countable and disjoint union of spaces $\mathcal{C}_{s}\left(\Gamma_{0}(N) \backslash \mathbb{H} ; \chi\right)$, as just introduced.

\footnotetext{
${ }^{(I)}$ For details about scaling matrices as just introduced cp. [14, Chapter 14, p.355] and [7 p.504].
} 
Remark 2.1. From this point onwards, for the remainder of this thesis, we are going to assume that the level $N$ of the Hecke congruence subgroup $\Gamma_{0}(N)$ is always an odd prime number. An important consequence of this assumption is that it immediately implies that all Dirichlet characters of modulus $N$, with the exception of the principal character $\chi_{0}$, are primitive.

There is one particular type of Maaß form that will also play an important role in the course of our investigations of this thesis. These functions are called Eisenstein series and are defined as

$$
E_{\mathfrak{a}}(z, s):=\sum_{\gamma \in \Gamma_{\mathfrak{a}} \backslash \Gamma_{0}(N)} \overline{\chi(\gamma)}\left(\Im\left(\sigma_{\mathfrak{a}}^{-1} \gamma z\right)\right)^{s}, \text { for } \Re(s)>1 .
$$

In the described half plane the series defining $E_{\mathfrak{a}}(z, s)$ converges absolutely. The Eisenstein series $E_{\mathfrak{a}}(z, s)$ is seen to be an element of $\mathcal{A}_{s}\left(\Gamma_{0}(N) \backslash \mathbb{H} ; \chi\right)$ and admits a meromorphic continuation to $\mathbb{C}$, which may be seen from [12, Theorem 11.6, p.128] and the generalisation of the theorem just quoted, from its treatment of groups with one cusp to the case of any finite number of cusps, given on [12, p.295-296]. The Eisenstein series does not possess any poles on the line $\Re(s)=1 / 2$ (cp. [12, Theorem 11.8, p.130]), for groups with one cusp, in combination with its generalisation to group with any finite number of cusp, given on [12, p.296-298]). Furthermore it may be developed into a Fourier expansion ${ }^{(\text {II) }}$ of the type

$$
E_{\mathfrak{a}}(z, s)=\delta_{\mathfrak{a}, \infty} y^{s}+\tau_{\mathfrak{a}, \chi}^{0}(s) y^{1-s}+y^{1 / 2} \sum_{m \in \mathbb{Z} \backslash\{0\}} \tau_{\mathfrak{a}, \chi}(m, s) K_{s-1 / 2}(2 \pi|m| y) e(m x),
$$

with the complex Fourier coefficients $\tau_{\mathfrak{a}, \chi}^{0}(s), \tau_{\mathfrak{a}, \chi}(m, s) \in \mathbb{C}$. For $s=1 / 2$ the expansion in 2.5$)$, as well as similar expressions in the remainder of this thesis, are to be interpreted as the limit as $s \rightarrow 1 / 2$.

\subsection{The Bruggeman-Kuznetsov formula}

In this section we are going to present a version of the well known Bruggeman-Kuznetsov formula. Broadly speaking it provides a link between a spectral side, featuring the Fourier coefficients of Eisenstein series, taken for instance from the expansion

$$
E_{\mathfrak{a}}(z, s)=\delta_{\mathfrak{a}, \infty} y^{s}+\tau_{\mathfrak{a}, \chi}^{0}(s) y^{1-s}+y^{1 / 2} \sum_{m \in \mathbb{Z} \backslash\{0\}} \tau_{\mathfrak{a}, \chi}(m, s) K_{s-1 / 2}(2 \pi|m| y) e(m x),
$$

as stated in 2.5, and the Fourier coefficients of Maaß cusp forms, from the space $\mathcal{C}\left(\Gamma_{0}(N) \backslash \mathbb{H} ; \chi\right)$, and an arithmetic side, essentially consisting of a sum over Kloosterman sums. For elements of the space of Maaß cusps forms, we may write their Laplace eigenvalue as $\lambda=(1-s) s$, for a complex $s=1 / 2+$ it with $t \in \mathbb{R} \cup(-\mathrm{i} / 2, \mathrm{i} / 2)$. Let $\mathcal{U}_{\chi}^{\mathrm{Maa} \beta}$ be an orthonormal basis for the space $\mathcal{C}\left(\Gamma_{0}(N) \backslash \mathbb{H}\right.$; $\left.\chi\right)$, where every member thereof admits a Fourier expansion of the type

$$
u(z)=y^{1 / 2} \sum_{m \in \mathbb{Z} \backslash\{0\}} \rho_{u}(m) K_{\mathrm{it}_{u}}(2 \pi|m| y) e(m x),
$$

(cp. (1.8) and (2.2)). A formula in the described spirit, connecting such a spectral side in the context of Maaß forms and an arithmetic side, was originally shown by Kuznetsov (cp. [16]) and independently Bruggeman (cp. [4]) for the first time. Over the years the Bruggeman-Kuznetsov formula has become very standard and in fact has been adapted to many different scenarios such as for instance different groups, mixed equivalence classes of cusps or general weight. The versions of the Bruggeman-Kuznetsov formula, that we are going to employ in this thesis, will be stated in the following.

\footnotetext{
(II) $\mathrm{cp}$. for $\Gamma_{0}(N)$ for instance for the character $\chi_{0}$ with the even more explicit form of the Fourier expansion as given in 13 Theorem 3.4] and for all other remaining (primitive) characters with [7 (5.3)] + Lemma 7.10
} 
Theorem 2.2 (Bruggeman-Kuznetsov formula). Let $h: \mathbb{C} \rightarrow \mathbb{C}$ be an even function which

1. admits at least the bound $h(z) \ll(1+|z|)^{-(2+\varepsilon)}$ and

2. is holomorphic in the strip $|\Im(z)| \leq 1 / 2+\varepsilon$.

For all positive integers $m, n \in \mathbb{Z}_{>0}$ it is true that

$$
\Xi_{\chi}^{\mathrm{disc}}(m, n)+\Xi_{\chi}^{\mathrm{cont}}(m, n)=\delta_{m, n} \int_{\mathbb{R}} h(w) d_{\mathrm{spec}} w+\phi_{\chi}(m, n),
$$

where we consider the integration with respect to the spectral measure defined as $d_{\mathrm{spec}} w:=w \tanh (\pi w) d w / \pi^{2}$. The two terms $\Xi_{\chi}^{\operatorname{disc}}(m, n)$ and $\Xi_{\chi}^{\mathrm{cont}}(m, n)$ on the spectral side of the formula denote respectively

$$
\sum_{u \in \mathcal{U}_{\chi}^{\text {Maa }}} \frac{\bar{\rho}_{u}(m) \rho_{u}(n)}{\cosh \left(\pi t_{u}\right)} h\left(t_{u}\right) \quad \text { and } \quad \sum_{\mathfrak{a}} \frac{1}{4 \pi} \int_{\mathbb{R}} \frac{\bar{\tau}_{\mathfrak{a}, \chi}(m, w) \tau_{\mathfrak{a}, \chi}(n, w)}{\cosh (\pi w)} h(w) d w
$$

and on the arithmetic side of the formula we define

$$
\phi_{\chi}(m, n):=\sum_{c \equiv 0 \bmod N} \frac{S_{\chi}(m, n ; c)}{c} \mathscr{J}\left(\frac{4 \pi \sqrt{m n}}{c}\right) .
$$

In the latter term we sum the integral transform

$$
\mathscr{J}(x):=2 \pi \mathrm{i} \int_{\mathbb{R}} \frac{J_{2 \mathrm{i} w}(x)}{\sinh (\pi w)} h(w) d_{\mathrm{spec}} w,
$$

where we integrate the product of the function $h$, a hyperbolic sine and the J-Bessel function, against the Kloosterman sum defined by

$$
S_{\chi}(m, n ; c):=\sum_{a \bmod c}^{*} \chi(a) e\left(\frac{a m+\bar{a} n}{c}\right),
$$

for a Dirichlet character $\chi$ of modulus $N$.

Proof. The following argument is mainly a collection of results from the literature, that upon combining will give a proof in support of the theorem. Since we assume that the level $N$ of the Hecke congruence subgroup, with respect to which we consider the Maaß forms in this context, is an odd prime number, as stated before any Dirichlet character modulo $N$ is thus primitive, unless we deal with the principal character. We will therefore treat in the following these two different cases separately, i.e. distinguishing whether the Dirichlet character $\chi$ is the principal character or a primitive one.

We start by dealing with the principal character $\chi_{0}$ first. In this case it is not hard to show that for any $\gamma \in \Gamma_{0}(N)$ we have that $\chi_{0}(\gamma)=1$. Also we see easily that for any invertible residue class $a(\bmod N l)$, for some $l \in \mathbb{Z}_{>0}$, we calculate that $\chi_{0}(a)=1$. Thus for the character $\chi_{0}$ the statement of Theorem 2.2 is the same as for the character $\chi \equiv 1$ and hence [14, Theorem 16.3] gives the statement of Theorem 2.2] in this case.

We are left to consider the case of the Dirichlet character $\chi$ being primitive. Form [10, 8.332.3] we know that $|\Gamma(1-\mathrm{i} x)|^{2}=\pi x / \sinh (\pi x)$, for $x \in \mathbb{R}$. Using the latter identity we quickly infer from [7] Proposition 
5.2] that for all $x \in \mathbb{R}$, all $m, n \in \mathbb{Z}_{>0}$, and every primitive Dirichlet character $\chi$ of odd prime modulus $N$ we have for weight $k=0$ that

$$
\begin{gathered}
\sum_{u \in \mathcal{U}_{\chi}^{\text {Maaß }}} \frac{\bar{\rho}_{u}(m) \rho_{u}(n)}{\cosh \left(\pi\left(x-t_{u}\right)\right) \cosh \left(\pi\left(x+t_{u}\right)\right)}+\sum_{\mathfrak{a}} \frac{1}{4 \pi} \int_{\mathbb{R}} \frac{\bar{\tau}_{\mathfrak{a}, \chi}(m, w) \tau_{\mathfrak{a}, \chi}(n, w)}{\cosh (\pi(x-w)) \cosh (\pi(x+w))} d w \\
=\frac{x}{\pi^{2} \sinh (\pi x)}\left(\delta_{m, n}+8 \mathrm{i} \pi \sqrt{m n} \sum_{c \equiv 0 \bmod N} \frac{S_{\chi}(m, n ; c)}{c^{2}} \int_{\mathscr{C}} K_{2 \mathrm{i} x}\left(\frac{4 \pi \sqrt{m n}}{c} \cdot t\right) \frac{d t}{t}\right)
\end{gathered}
$$

where we integrate in the clockwise direction along the contour $\mathscr{C}$, which connects with a circle of radius one centred at the origin the points $-\mathrm{i}$ and $\mathrm{i}$ and where $K$ denotes the corresponding Bessel function. In order to obtain (2.8) from [7, Proposition 5.2] we have employed Lemma 7.10 to be able to renormalise the Fourier coefficients of the Maaß cusp forms and the Eisenstein series to our setup as introduced in Section 2.2 (cp. [7, (5.1) and (5.3)] with (1.8) and (2.5) respectively). Thus we now arrive at Theorem 2.2 with the same argument given in support of [14, Theorem 16.3; see p.409 for the statement and p.409-410 for its proof], where we use [2.8 instead of [14, (16.32)]. Upon merging the two described cases we obtain the result.

In the introduction we announced that in this thesis we will consider a family of Maaß cusp forms with more elements than in an orthonormal basis for $\mathcal{C}\left(\Gamma_{0}(N) \backslash \mathbb{H} ; \chi\right)$. We do this by dealing with several of these spaces for different Dirichlet characters $\chi$ simultaneously. In particular our new enlarged family is going to be

$$
\mathcal{U}^{\mathrm{Maa} \beta}:=\bigcup_{\substack{\chi \bmod N \\ \chi(-1)=1}} \mathcal{U}_{\chi}^{\mathrm{Maa}} .
$$

We point out that the condition that we only consider even characters prevents us from taking into account such spaces that merely contain the zero function. For the set of harmonics $\mathcal{U}^{\mathrm{Ma} B}$ we need a suitable version of the Bruggeman-Kuznetsov formula. The following presentation is a direct consequence of Theorem 2.2 . derived by simply summing 2.6 over all even characters and then normalising both sides by dividing by their total number, which is $\varphi(N) / 2$.

Theorem 2.3. Let $h: \mathbb{C} \rightarrow \mathbb{C}$ be a function satisfying the same conditions as imposed on it in Theorem 2.2. Then again for $m, n \in \mathbb{Z}_{>0}$ we find that

$$
\Xi^{\operatorname{disc}}(m, n)+\Xi^{\text {cont }}(m, n)=\delta_{m, n} \int_{\mathbb{R}} h(w) d_{\text {spec }} w+\phi(m, n) .
$$

In this context the spectral side of the formula features the two components $\Xi^{\text {disc }}(m, n)$ and $\Xi^{\operatorname{cont}}(m, n)$ representing respectively the two sums

$$
\frac{2}{\varphi(N)} \sum_{u \in \mathcal{U}_{\text {Maaß }}} \frac{\bar{\rho}_{u}(m) \rho_{u}(n)}{\cosh \left(\pi t_{u}\right)} h\left(t_{u}\right) \text { and } \frac{2}{\varphi(N)} \sum_{\chi \bmod N}^{+} \sum_{\mathfrak{a}} \frac{1}{4 \pi} \int_{\mathbb{R}} \frac{\bar{\tau}_{\mathfrak{a}, \chi}(m, w) \tau_{\mathfrak{a}, \chi}(n, w)}{\cosh (\pi w)} h(w) d w,
$$

whereas on the arithmetic side we encounter now the term

$$
\phi(m, n):=\sum_{c \equiv 0 \bmod N} \frac{\mathcal{S}(m, n ; c)}{c} \mathscr{J}\left(\frac{4 \pi \sqrt{m n}}{c}\right),
$$

In the latter sum the integral transform $\mathscr{J}$ is just as in (2.7), which is in this case summed against a Kloosterman sum, containing more terms than before and that we define by

$$
\mathcal{S}(m, n ; c):=\frac{2}{\varphi(N)} \sum_{\chi \bmod N}^{+} S_{\chi}(m, n ; c) .
$$


The latter result given as Theorem 2.3 is the staring point of our considerations in the upcoming chapters, upon which all of our results hinge. We remark that there is a set of requirements the functions $h$, as introduced in Theorem 2.2 has to fulfil in order for the two theorems given in this section to be valid, we are in fact going to refine the class of functions we will work with in subsequent chapters. Giving a precise description of this particular family of functions is going to be the subject of the next section.

\subsection{Schwartz functions}

From now on in this thesis we will consider only such functions $h: \mathbb{C} \rightarrow \mathbb{C}$, required by the BruggemanKuznetsov formulae as introduced in Section 2.3, that are of the form

$$
h(z)=\left(z^{2}+1 / 4\right) h^{*}(z), \text { where } h^{*} \in \mathcal{S}(\mathbb{C})
$$

is a Schwartz function, such that

1. $h$ is holomorphic in the strip $|\Im(z)| \leq 1 / 2+\varepsilon$, for $\varepsilon>0$,

2. $h$ satisfies $\left.h\right|_{\mathbb{R}}=\left.\bar{h}\right|_{\mathbb{R}}$

3. $h$ is an even function

4. and we have a bound $h(z) \ll \exp (-100|z|)$.

Thus in particular we have that the function $h \in \mathcal{S}(\mathbb{C})$ is also a Schwartz function and clearly fulfils the requirements demanded for functions in the Bruggeman-Kuznetsov formulae, specified in Theorem 2.2 .

Remark 2.4. We point out that since $h$ is a Schwartz function, as specified above, shifting the contour of integration in the integral (2.7), the decay properties of the function ensure that for $x \in \mathbb{R}_{>0}$ we may always write

$$
\mathscr{J}(x)=\frac{2 \mathrm{i}}{\pi} \int_{\mathbb{R}} \frac{J_{2 \mathrm{i} z \pm c}(x)(z \pm \mathrm{i} c / 2)}{\cosh (\pi(z \pm \mathrm{i} c / 2))} h(z \pm \mathrm{i} c / 2) d z
$$

where we shifted the contour to $|\Im(z)|=c / 2$, for some $0 \leq c<1+2 \varepsilon$.

Remark 2.5. Furthermore in particular the bound demanded above for the function $h$ guarantees that an integral of the form

$$
\int_{\mathbb{R}} \exp (C|z|)|h(z)| d z \ll 1
$$

converges absolutely, for any fixed choice of a constant $0<C<100$. The implied constant of course depends always on the function $h$ itself.

\subsection{The Riemann hypothesis}

We are briefly going to discuss the Riemann hypothesis $(\mathrm{RH})$ for the Riemann zeta function and Dirichlet $L$-functions. The Riemann zeta function is defined by

$$
\zeta(s)=\sum_{n=1}^{\infty} \frac{1}{n^{s}}, \text { for } \Re(s)>1 .
$$


For instance from [14, Theorem 4.15] we know that the Riemann zeta function admits a meromorphic continuation to $\mathbb{C}$ and possesses at $s=1$ a pole of order one. It does not have any further poles. For any Dirichlet character $\chi$ of modulus $N$ we define the Dirichlet $L$-function corresponding to it by

$$
L(s, \chi)=\sum_{n=1}^{\infty} \frac{\chi(n)}{n^{s}}, \text { for } \Re(s)>1 .
$$

Now if we consider the principal character $\chi_{0}$ of modulus $N$, then, again for $\Re(s)>1$, we have the relationship

$$
L\left(s, \chi_{0}\right)=\zeta(s) \prod_{p \mid N}\left(1-\frac{1}{p^{s}}\right)
$$

between the corresponding $L$-function and the Riemann zeta function (cp. [3, p.35]). Since the finite product in 2.13) is an entire function of the variable $s$, from the meromorphic continuation of $\zeta$ to $\mathbb{C}$, we have that $L\left(s, \chi_{0}\right)$ has also meromorphic continuation to the complex plane, with the same pole as the Riemann zeta function. Now if we exclude the principal character, i.e $\chi \neq \chi_{0}$, we further know for instance with [3, Satz 1.6.1] that the corresponding Dirichlet $L$-functions defined by the series in (2.12) converges even for $\Re(s)>0$. A typical formulation of the RH makes a prediction about the location of zeros of the functions $L(s, \chi)$ and $\zeta(s)$ in the strip $0<\Re(s)<1$.

The Riemann hypothesis 2.6 (Version I). Let $\zeta(s)$ be the Riemann zeta function and $L(s, \chi)$ be a Dirichlet series for any Dirichlet character $\chi$ of modulus $N$. If we encounter that in the strip $0<\Re(s)<1$ any of the two functions has a zero, i.e.

$$
\zeta\left(s^{*}\right)=0 \quad \text { or } L\left(s^{*}, \chi\right)=0
$$

then this implies that $\Re\left(s^{*}\right)=1 / 2$.

By a standard Perron style argument one can show that Version I of the RH is in fact equivalent to the following formulation.

The Riemann hypothesis 2.7 (Version II). Let $\chi$ be any Dirichlet character of modulus $N$ and $\mu$ denotes the Möbius function. Then we have the bounds

$$
\sum_{k \leq X} \mu(k) \ll X^{1 / 2+\varepsilon} \quad \text { and } \quad \sum_{k \leq X} \mu(k) \chi(k) \ll N^{\varepsilon} X^{1 / 2+\varepsilon}
$$

for any $\varepsilon>0$.

Up to this day the Riemann hypothesis remains unproven. However it is widely believed to be true. 


\section{Chapter 3}

\section{An asymptotic expansion for the Bruggeman-Kuznetsov formula}

\subsection{The main theorem}

The goal of this chapter is to develop an asymptotic formula for the sum of $\Xi^{\operatorname{disc}}(m, n)$ and $\Xi^{\text {cont }}(m, n)$ as introduced in Theorem 2.3. To this end will investigate the term $\phi(m, n)$ and split it into appropriate main and error term. As a result the following theorem will be established.

Theorem 3.1. Assume $N$ is an odd prime. Let $m, n \in \mathbb{N}$ and $\Xi^{(\cdots)}(m, n)$ be as in Theorem 2.3 . Furthermore consider any smooth even function $f: \mathbb{R} \rightarrow \mathbb{R}_{\geq 0}$ with $\operatorname{supp}(f) \subseteq[-2,2]$, which equals one on $[-1,1]$. Now for any choice of $\theta \in[0,1)$ we have

$$
\Xi^{\mathrm{disc}}(m, n)+\Xi^{\mathrm{cont}}(m, n)=\delta_{m, n} \int_{\mathbb{R}} h(w) d_{\mathrm{spec}} w+\phi_{f}(m, n)-\phi_{f}^{0}(m, n)+\mathcal{O}\left(\frac{1}{N}\left(\frac{N}{m+n}\right)^{\theta}\right),
$$

with the two main terms being defined respectively as

$$
\phi_{f}(m, n):=\frac{1}{N} \sum_{t=1}^{\infty} \frac{1}{t^{2}} \sum_{h=-\infty}^{\infty} f\left(\frac{h}{H}\right) \sum_{\substack{a \bmod t \\ a N \equiv h \bmod t}} S(a, m, t) S(a, n, t) \mathscr{I}\left(\frac{m}{N t}, \frac{n}{N t}, \frac{h}{t}\right)
$$

and

$$
\phi_{f}^{0}(m, n):=\frac{1}{N} \sum_{t=1}^{\infty} \frac{S(0, m-n, t)}{t^{2}} \int_{\mathbb{R}} f\left(\frac{y}{H}\right) \mathscr{I}\left(\frac{m}{N t}, \frac{n}{N t}, \frac{y}{t}\right) d y .
$$

In the latter two main terms the cut-off parameter $H$, which we use in the smooth truncation of the $h$ summation and the $y$ integral respectively, is considered to be a function of the summation variable $t$. Furthermore we require for it that $H \geq \max \left\{2 t+81(m+n) / N, t^{B}\right\}$, for a $B>3 /(1-\theta)$. The function $\mathscr{I}$ is defined as follows. Let its first and second argument be as is the statement above and assume the third argument is $r / t$, for any $r \in \mathbb{R}$. Then in the case of $r>0$ the function $\mathscr{I}$ is

$$
-\mathrm{i} \int_{\mathbb{R}} \frac{4 \pi^{2} h(x)}{\sinh ^{2}(\pi x)} J_{2 \mathrm{i} x}\left(\frac{4 \pi}{t} \sqrt{\frac{r m}{N}}\right) \Im\left(J_{2 \mathrm{i} x}\left(\frac{4 \pi}{t} \sqrt{\frac{r n}{N}}\right)\right) d_{\mathrm{spec}} x .
$$


If in the third variable we encounter $r<0$, it is

$$
\mathrm{i} \int_{\mathbb{R}} \frac{8 \pi h(x)}{\tanh (\pi x)} Y_{2 \mathrm{i} x}\left(\frac{4 \pi}{t} \sqrt{\frac{|r| \max \{m, n\}}{N}}\right) K_{2 \mathrm{i} x}\left(\frac{4 \pi}{t} \sqrt{\frac{|r| \min \{m, n\}}{N}}\right) d_{\text {spec }} x
$$

and if the last variable equals zero, i.e. $r=0$, then $\mathscr{I}$ becomes

$$
\frac{2}{\pi} \int_{\mathbb{R}} h(x)\left(\frac{\sqrt{m n}}{\max \{m, n\}}\right)^{2 \mathrm{i} x} d x
$$

Here we denote by $J, Y$ and $K$ the corresponding Bessel functions. We highlight the fact that the function $h$ is assumed to admit the conditions as introduced in Section 2.4

The theorem provides in some sense an asymptotic Kuznestov formula for a larger family of Maaß cusp forms. Theorem 3.1 is the non-holomorphic equivalent of [15, Theorem 3.1] and indeed both results share some similarities. The main terms in both theorems are in fact fairly similar in style, however mild differences occur due to the fact that we are working with the non-holomorphic set up of the BruggemanKuznetsov formula that equates the sum of sums over Fourier coefficients of a family of Maaß forms and Fourier coefficients of Eisenstein series to a sum over Kloosterman sums. Before we turn our attention in the following chapters towards developing an asymptotic expansion further in the context of quadratic forms, we will devote the rest of this chapter to giving a proof of the result just stated. As a byproduct of these considerations we will also develop a preliminary result, Theorem 3.2, which is going to turn out to be very useful and in fact our arguments in subsequent chapters will hinge on this theorem. On the other hand the sole purpose of Theorem 3.1 is to give a description of the Bruggeman-Kuznetsov formula, however we won't have to rely on it showing the results of the following chapters.

\subsection{Proof of the main theorem}

In the remainder of this chapter we are going to give a proof of Theorem 3.1. Our whole argument follows closely the details and structure of the argument presented in [15, Chapters $2 \& 3$ (p. 543-550)], however for the sake of a coherent account of our results in this thesis and maybe more importantly the fact that we have to adapt the argument slightly to our situation in the context of Maaß cusp forms we retrace the steps of the proof here carefully.

For the remainder of this chapter we assume that $N$ is an odd prime number. In order to establish Theorem 3.1. we begin by considering the right hand side of (2.9) in the Bruggeman-Kuznetsov formula as given in Theorem 2.3. In particular we will start to manipulate

$$
\phi(m, n)=\sum_{c \equiv 0 \bmod N} \frac{\mathcal{S}(m, n ; c)}{c} \mathscr{J}\left(\frac{4 \pi \sqrt{m n}}{c}\right)
$$

the sum over Kloosterman sums, and develop it into an asymptotic expansion. We want to simplify the notation and thus introduce the operator $\mathrm{P}(g)=2 \Re(g)$, acting on functions $g: \mathbb{C} \rightarrow \mathbb{C}$, which immediately allows us to express the Kloosterman sum in 2.11) as

$$
\mathcal{S}(m, n ; c)=\mathrm{P} \sum_{\substack{a \bmod c \\ a \equiv 1 \bmod N}}^{*} e\left(\frac{a m+\bar{a} n}{c}\right) .
$$

We highlight the fact that we only sum over such residue classes for which $a \equiv 1(\bmod N)$, which resonates the orthogonality condition

$$
\sum_{\chi \bmod N} \chi(a)= \begin{cases}\varphi(N), & \text { if } a \equiv 1(\bmod N) \\ 0, & \text { otherwise }\end{cases}
$$


for Dirichlet characters $\chi$ of modulus $N$, that we used to obtain the Kloosterman sum in the described form. We proceed by rewriting the summation over the residue classes in 3.2 . The resulting description will be more feasible to work with and is exactly the same as introduced in [15, Chapter 2: A trace formula for the Fourier coefficients of cusp forms, p.545]: To this end let $c=N r, a=1+N q$, where $q$ is a residue class of modulus $r$ and call $\operatorname{gcd}(q, r)=y$ and $r=y t$, for an appropriate $t$. Then for a residue class $x$ of modulus $t$ we arrive at the formulation $a=1+N y x$ with $\operatorname{gcd}(x(1+N y x), t)=1$. The latter gcd condition is satisfied since we consider only invertible residue classes $a$ of modulus $c$ to begin with. We define the exponential sum

$$
\mathscr{S}_{N y}(m, n, t):=\sum_{\substack{x \bmod t \\(x(1+N y x), t)=1}} e\left(\frac{\bar{x} m-n \overline{(x+N y)}}{t}\right),
$$

and then, using the new formulation just introduced, the Kloosterman sum becomes

$$
\mathcal{S}(m, n ; c)=\mathrm{P}\left(e\left(\frac{m+n}{c}\right) \sum_{N y t=c} \mathscr{S}_{N y}(m, n, t)\right) .
$$

By a change of variables we see that the integral in 3.1 satisfies $\left.\mathscr{J}\right|_{\mathbb{R}}=\left.\overline{\mathscr{J}}\right|_{\mathbb{R}}$. This enables us to move it past the operator $\mathrm{P}$, for which we highlight the simple observation that by its definition it admits $\mathrm{P}(r g)=r \mathrm{P}(g)$, whenever $r \in \mathbb{R}$. Thus inserting the latest formulation of the Kloosterman sum into 3.1) gives us

$$
\phi(m, n)=\mathrm{P} \sum_{y=1}^{\infty} \sum_{t=1}^{\infty} \frac{\mathscr{S}_{N y}(m, n, t)}{N y t} e\left(\frac{m+n}{N y t}\right) \mathscr{J}\left(\frac{4 \pi \sqrt{m n}}{N y t}\right)
$$

This is an important formulation that will be useful in certain situations in upcoming chapters to estimate parts of $\phi(m, n)$. At this point however we simplify the notation slightly further and continue our considerations by first treating the sums without the $t$-summation. To this end we let

$$
\phi(m, n)=\sum_{t=1}^{\infty} \frac{\phi(m, n, t)}{N t}
$$

with the definition

$$
\phi(m, n, t):=\mathrm{P} \sum_{y=1}^{\infty} \frac{\mathscr{S}_{N y}(m, n, t)}{y} e\left(\frac{m+n}{N y t}\right) \mathscr{J}\left(\frac{4 \pi \sqrt{m n}}{N y t}\right) .
$$

Keeping the split in 3.5 for $\phi(m, n)$ in mind, we at once establish Theorem 3.1 upon combining the Bruggeman-Kuznestov formula as stated in Theorem 2.3 with the following result.

Theorem 3.2. Let $m, n \in \mathbb{N}$. For a cut-off parameter $H \geq 2 t+81(m+n) / N$, for $N$ an odd prime, we find that

$$
\phi(m, n, t)=\phi_{f}(m, n, t)-\phi_{f}^{0}(m, n, t)+\phi_{\text {error }}(m, n, t),
$$

where the two main terms are given respectively by

$$
\phi_{f}(m, n, t):=\frac{1}{t} \sum_{h=-\infty}^{\infty} f\left(\frac{h}{H}\right) \sum_{\substack{a \bmod t \\ a N \equiv h \bmod t}} S(a, m, t) S(a, n, t) \mathscr{I}\left(\frac{m}{N t}, \frac{n}{N t}, \frac{h}{t}\right)
$$


and

$$
\phi_{f}^{0}(m, n, t):=\frac{S(0, m-n, t)}{t} \int_{\mathbb{R}} f\left(\frac{y}{H}\right) \mathscr{I}\left(\frac{m}{N t}, \frac{n}{N t}, \frac{y}{t}\right) d y .
$$

In both terms $f: \mathbb{R} \rightarrow \mathbb{R}_{\geq 0}$ is any smooth even cut-off function with $\operatorname{supp}(f) \subseteq[-2,2]$, that takes the value one on the interval $[-1,1]$. The error term satisfies

$$
\phi_{\text {error }}(m, n, t) \ll t H\left(\frac{t}{H}\right)^{j},
$$

for any $j \geq 2$. For completeness we highlight that the function $\mathscr{I}$ is just as specified in Theorem 3.1 . varying significantly in its shape depending on the values of third variable.

The result we just introduced will also prove useful later, since by the split in $(3.5)$ the theorem provides in some sense almost an asymptotic for the term $\phi(m, n)$, however without yet taking the $t$-summation into consideration. This particular feature will allow for the necessary flexibility in Chapter 4 , where we are going to estimate the terms for certain ranges of the $t$-summation. The remainder of this chapter is devoted to proving Theorem 3.2, which in turn will then directly settle Theorem 3.1. upon employing the inequality

$$
\max \{A, B\} \geq A^{1-\theta} B^{\theta},
$$

for any $A, B \in \mathbb{R}_{>0}$ and any $\theta \in[0,1]$, to lower bound the cut-off parameter $H$, when establishing the error term. The argument supporting Theorem 3.2 will essentially be divided into two parts. First we are going to cut smoothly the $h$-summation, corresponding to the $y$ sum in (3.6). This will give rise to a main term, essentially containing the terms for which the values of $h$ are in a way small, and an error term for large $h$. The error term resulting from separating the $h$-summation already reflects the right magnitude as the bound for $\phi_{\text {error }}(m, n, t)$ in Theorem 3.2 In a second step we will then modify the main term we encounter for small $h$. Removing a smoothing function, introduced for technical reasons, we are going to end up with two main terms finally resulting in $\phi_{f}(m, n, t)$ and $\phi_{f}^{0}(m, n, t)$, as given in the theorem. We will now execute this strategy in more detail in the following.

\subsubsection{A preliminary asymptotic}

As previously described in the plan for the rest of the argument for Theorem 3.2, in this subsection we are going to establish a first asymptotic for $\phi(m, n, t)$ and then specify the size of its error term. The starting point here is of course 3.6 . We introduce a smooth function $\varrho: \mathbb{R}_{>0} \rightarrow[0,1]$, with $\operatorname{supp}(\varrho) \subseteq[1 / 3, \infty)$ and that equals one on $(1 / 2, \infty)$, which, with argument $y$, we simply insert into 3.6 without changing $\phi(m, n, t)$ in any way. We observe that the exponential sum $\mathscr{S}_{N y}(m, n, t)$ depends in the $y$ variable only on residue classes of modulus $t$. Thus reordering the $y$-summation in $\phi(m, n, t)$ into such residue classes, we see that the latter becomes

$$
\mathrm{P} \sum_{z \bmod t} \mathscr{S}_{N z}(m, n, t) \sum_{y \equiv z \bmod t} \frac{\varrho(y)}{y} e\left(\frac{m+n}{N y t}\right) \mathscr{J}\left(\frac{4 \pi \sqrt{m n}}{N y t}\right) .
$$

Now we apply Poisson summation to the $y$ sum, after which the previous expression equals

$$
\mathrm{P} \sum_{h=-\infty}^{\infty} \frac{\mathscr{W}(h, m, n, t)}{t} \int_{0}^{\infty} \varrho(s) e\left(\frac{h s}{t}+\frac{m+n}{N s t}\right) \mathscr{J}\left(\frac{4 \pi \sqrt{m n}}{N s t}\right) \frac{d s}{s},
$$


where we simplify notation by denoting

$$
\mathscr{W}(h, m, n, t):=\sum_{z \bmod t} \mathscr{S}_{N z}(m, n, t) e\left(\frac{-h z}{t}\right) .
$$

Since $\mathscr{W}$ is an exponential sum, where the summation runs over residue classes of modulus $t$, by a reordering of the summation we see that in fact $\mathscr{W}=\overline{\mathscr{W}}$, the property that in a moment will again enable us to move it past the operator P. This will become useful, when splitting the $h$-summation in $\sqrt{3.8}$ into a part of large absolute summation index and a part of small one. This is achieved by introducing a smooth even cut-off function $f: \mathbb{R} \rightarrow \mathbb{R}_{\geq 0}$ with $\operatorname{supp}(f) \subseteq[-2,2]$ and that equals one on the whole interval $[-1,1]$. Abbreviating

$$
G(h, m, n, t):=\frac{\mathscr{W}(h, m, n, t)}{t} \mathrm{P} \int_{0}^{\infty} \varrho(s) e\left(\frac{h s}{t}+\frac{m+n}{N s t}\right) \mathscr{J}\left(\frac{4 \pi \sqrt{m n}}{N s t}\right) \frac{d s}{s},
$$

we now divide the $h$-summation essentially at some cut-off parameter $H$, whose size we are going to specify shortly, and thus obtain

$$
\phi(m, n, t)=\sum_{h=-\infty}^{\infty} G(h, m, n, t) f\left(\frac{h}{H}\right)+\phi_{\text {error.1 }}(m, n, t),
$$

where the part of the sum for large absolute summation index is given by

$$
\phi_{\text {error.1 }}(m, n, t):=\sum_{H<|h| \leq 2 H} G(h, m, n, t)\left(1-f\left(\frac{h}{H}\right)\right)+\sum_{2 H<|h|} G(h, m, n, t) .
$$

To establish 3.10 as a first version of some sort of an asymptotic formula for $\phi(m, n, t)$ we have to identify the error and respectively main term herein. It turns out that the term $\phi_{\text {error. }}(m, n, t)$ is indeed relatively small and the terms of the $h$-summation for small absolute summation index will make up the main term. This behaviour is explained be the following lemma, which characterises the size of the integral in 3.8.

Lemma 3.3. Let $|h| \geq 81(m+n) / N$, then we have

$$
\int_{0}^{\infty} \varrho(s) e\left(\frac{h s}{t}+\frac{m+n}{N s t}\right) \mathscr{J}\left(\frac{4 \pi \sqrt{m n}}{N s t}\right) \frac{d s}{s} \ll\left(1+\frac{|h|}{t}\right)^{-j},
$$

for every $j \geq 0$. The bound is uniform in $m, n, N, t$.

We are going to provide a full proof supporting this result in Section 3.3 . However we still point out that introducing the smooth function $\varrho$ earlier in this section is necessary for technical reasons to be able to show this lemma (cp. Remark 3.4]; With this ansatz we follow the one used in [15, Chapter 3: An asymptotic trace formula]. A discussion of the details and implications will follow at a later stage. For now we focus on employing the lemma in the course of our current argument. We proceed by highlighting several aspects of it. First of all the condition $|h| \geq 81(m+n) / N$ is necessary to avoid that the first derivative of the phase function of the exponential in 3.12 is equal to zero. This includes the oscillating part of the $J$-Bessel function, contained in the integral $\mathscr{J}$, which we will identify and extract by means of Lemma 7.7 Having no stationary phase the integral can now be estimated using integration by parts repeatedly, exploiting the oscillation of the function we integrate. Thus for $|h| / t>1$ from Lemma 3.3 we get an estimate of the oscillatory integral described therein, whose magnitude may be controlled to our liking. In particular we choose

$$
H \geq 2 t+81(m+n) / N
$$


which means that in both of the $h$-summations in $\phi_{\text {error.1 }}(m, n, t)$ we find ourselves in a scenario where Lemma 3.3 tells us that the integrals contained herein are small, causing the whole error term to be of moderate size. Indeed, using that the cut-off function $f$ is uniformly bounded from above by a constant, from (3.11) we conclude with Lemma 3.3 that $\phi_{\text {error. } 1}(m, n, t)$ is dominated by

$$
\sum_{H<|h|}|G(h, m, n, t)| \ll \sum_{H<|h|} \frac{1}{t} \sum_{z \bmod t}\left|\mathscr{S}_{N z}(m, n, t)\right|\left(\frac{|h|}{t}\right)^{-j} .
$$

Recalling the definition of the exponential sum $\mathscr{S}_{N z}(m, n, t)$ in $\sqrt{3.3}$, we find further that

$$
\phi_{\text {error.1 }}(m, n, t) \ll t \sum_{H<h}\left(\frac{h}{t}\right)^{-j}=t^{j+1} \sum_{i=1}^{\infty} \sum_{i H<h \leq(i+1) H} h^{-j} \ll t H\left(\frac{t}{H}\right)^{j},
$$

where we require that $j \geq 2$, in order to ensure convergence of the $i$ sum involved.

\subsubsection{Calculating the main terms}

This section is devoted to dealing with the main term in 3.10 , given by

$$
\frac{1}{t} \sum_{h=-\infty}^{\infty} f\left(\frac{h}{H}\right) \mathscr{W}(h, m, n, t) \mathrm{P} \int_{0}^{\infty} \varrho(s) e\left(\frac{h s}{t}+\frac{m+n}{N s t}\right) \mathscr{J}\left(\frac{4 \pi \sqrt{m n}}{N s t}\right) \frac{d s}{s} .
$$

The main objective is to remove the smooth function $\varrho$ in the integral of the above expression. The necessity for introducing this function is of technical nature and will become more apparent in Section 3.3, where we are going to provide further explanations. The reason for removing this function lies in the fact that the resulting integral

$$
\mathrm{P} \int_{0}^{\infty} e\left(\frac{h s}{t}+\frac{m+n}{N s t}\right) \mathscr{J}\left(\frac{4 \pi \sqrt{m n}}{N s t}\right) \frac{d s}{s}
$$

is essentially an integral over the Hankel transform of the cosine function ${ }^{(\mathrm{I})}$, with the transform being computable explicitly, depending on the value of $h$. As a result we are going to obtain integrals over Bessel functions, which will prove useful in subsequent estimations, and an oscillatory integral which in some sense is an echo of the term $\Xi^{\text {cont }}(m, n)$, as defined in Theorem 2.3 , on the arithmetic side of the Bruggeman-Kuznetsov formula. We will treat and highlight these connections in more detail as we proceed in the following chapters. At this point we are actually going to remove $\varrho$. To do this we define $\lambda(x):=$ $1-\varrho(x)$, which satisfies $\operatorname{supp}(\lambda) \subseteq[0,1 / 2]$. Thus the expression in 3.15 is actually equal to

$$
\widetilde{\phi_{f}}(m, n, t)-\widetilde{\phi_{f}^{0}}(m, n, t) \text {, }
$$

where we define

$$
\widetilde{\phi_{f}}(m, n, t):=\frac{1}{t} \sum_{h=-\infty}^{\infty} f\left(\frac{h}{H}\right) \mathscr{W}(h, m, n, t) \mathrm{P} \int_{0}^{\infty} e\left(\frac{h s}{t}+\frac{m+n}{N s t}\right) \mathscr{J}\left(\frac{4 \pi \sqrt{m n}}{N s t}\right) \frac{d s}{s}
$$

and

$$
\widetilde{\phi_{f}^{0}}(m, n, t):=\frac{1}{t} \sum_{h=-\infty}^{\infty} f\left(\frac{h}{H}\right) \mathscr{W}(h, m, n, t) \mathrm{P} \int_{0}^{1 / 2} \lambda(s) e\left(\frac{h s}{t}+\frac{m+n}{N s t}\right) \mathscr{J}\left(\frac{4 \pi \sqrt{m n}}{N s t}\right) \frac{d s}{s} .
$$

\footnotetext{
(I) Essentially using that $\mathrm{P}(e(x))=2 \cos (2 \pi x)$.
} 
The goal is now to rearrange the above terms and extract the two main terms $\phi_{f}(m, n, t)$ and $\phi_{f}^{0}(m, n, t)$ respectively from them. The latter are then the main terms in the asymptotic claimed in Theorem 3.2 . We remark that these considerations show us, that essentially the price we have to pay for introducing $\varrho$ in the first place is the second main term $\phi_{f}^{0}(m, n, t)$, which is the result of our attempts to remove this function again. Since manipulating $\widetilde{\phi_{f}}(m, n, t)$ into the desired form is relatively simple we begin with it. From [15, Lemma 3.1] we know that

$$
\mathscr{W}(h, m, n, t)=\sum_{\substack{a \bmod t \\ a N \equiv h \bmod t}} S(a, m, t) S(a, n, t),
$$

for $m, n, h \in \mathbb{Z}$ and $t \in \mathbb{N}(I I)$. Thus working towards the form of the first main term defined in $\phi_{f}(m, n, t)$, after replacing the function $\mathscr{W}$ in 3.17 by the sum over a product of Kloosterman sums as just stated above, it remains to treat the integral in the expression we are currently working with. Employing the definition in 7.12 this integral is then equal to the function $\mathscr{I}(m / N t, n / N t, h / t)$, for which Lemma 7.6 tells us that this integral over a Hankel transform is explicitly

$$
-\mathrm{i} \int_{\mathbb{R}} \frac{4 \pi^{2} h(x)}{\sinh ^{2}(\pi x)} J_{2 \mathrm{i} x}\left(\frac{4 \pi}{t} \sqrt{\frac{h m}{N}}\right) \Im\left(J_{2 \mathrm{i} x}\left(\frac{4 \pi}{t} \sqrt{\frac{h n}{N}}\right)\right) d_{\mathrm{spec}} x,
$$

provided the summation variable $h$ is positive, it is equal to

$$
\mathrm{i} \int_{\mathbb{R}} \frac{8 \pi h(x)}{\tanh (\pi x)} Y_{2 \mathrm{i} x}\left(\frac{4 \pi}{t} \sqrt{\frac{h \max \{m, n\}}{N}}\right) K_{2 \mathrm{i} x}\left(\frac{4 \pi}{t} \sqrt{\frac{h \min \{m, n\}}{N}}\right) d_{\text {spec } x}
$$

in the case of $h<0$, and it takes for $h=0$ the form

$$
\frac{2}{\pi} \int_{\mathbb{R}} h(x)\left(\frac{\sqrt{m n}}{\max \{m, n\}}\right)^{2 \mathrm{i} x} d x
$$

Thus we conclude indeed that $\widetilde{\phi_{f}}(m, n, t)=\phi_{f}(m, n, t)$. After having sorted out the first main term of the asymptotic expansion in Theorem 3.2. we now turn our attention to the second one, which is $\widetilde{\phi_{f}^{0}}(m, n, t)$. Applying Poisson summation to the $h$ sum, 3.18 becomes

$$
\sum_{z \bmod t} \frac{\mathscr{S}_{N z}(m, n, t)}{t} \mathrm{P} \int_{0}^{1 / 2} H \sum_{k=-\infty}^{\infty} \hat{f}\left(H\left(k+\frac{z-s}{t}\right)\right) \lambda(s) e\left(\frac{m+n}{N s t}\right) \mathscr{J}\left(\frac{4 \pi \sqrt{m n}}{N s t}\right) \frac{d s}{s},
$$

where we have inserted the original definition of the function $\mathscr{W}$ as an exponential sum as in 3.9p. In the last expression the involved Fourier transform is seen by a repeated integration by parts argument to admit for every $j \geq 0$ the bounds $\hat{f}(z) \ll(1+|z|)^{-j}$. This in turn tells us that as soon as we encounter $|z|>1$ the Fourier transform is going to be very small, and is bounded by a constant otherwise. In our scenario for most of the terms we face $z \not \equiv 0(\bmod t)$, which means $z / t \notin \mathbb{Z}$ and so there is no summation index in the $k$ sum adding together with the term $z / t$ up to zero. Indeed we can then show that uniformly for all variables involved we have

$$
\left|H\left(k+\frac{z-s}{t}\right)\right|>1,
$$

\footnotetext{
${ }^{(\text {II) With the above notation the Kloosterman } \operatorname{sum} S}(a, m, t)=S_{\chi \equiv 1}(a, m, t)$.
} 
in light of the lower bound 3.13 for the parameter $H$. As explained above, in that case the Fourier transform is then small. We extract such terms in (3.19), by splitting the latter into

$$
\begin{aligned}
\frac{S(0, m-n, t)}{t} \mathrm{P} \int_{0}^{1 / 2} H \hat{f}\left(\frac{H s}{t}\right) \lambda(s) e\left(\frac{m+n}{N s t}\right) \mathscr{J} & \left(\frac{4 \pi \sqrt{m n}}{N s t}\right) \frac{d s}{s} \\
& +\phi_{\text {error.2 }}(m, n, t)+\phi_{\text {error. } 3}(m, n, t)
\end{aligned}
$$

where we have used that the Fourier transform $\hat{f}$ is an even function, since $f$ is assumed to be an even function itself. The terms $\phi_{\text {error. } 2}(m, n, t)$ and $\phi_{\text {error. } 3}(m, n, t)$ represent cases in which the Fourier transform is small. The first error term $\phi_{\text {error.2 }}(m, n, t)$ is given by

$$
\sum_{\substack{z \bmod t \\ z \neq 0 \bmod t}} \frac{\mathscr{S}_{N z}(m, n, t)}{t} \mathrm{P} \int_{0}^{1 / 2} H \sum_{k=-\infty}^{\infty} \hat{f}\left(H\left(k+\frac{z-s}{t}\right)\right) \lambda(s) e\left(\frac{m+n}{N s t}\right) \mathscr{J}\left(\frac{4 \pi \sqrt{m n}}{N s t}\right) \frac{d s}{s},
$$

whereas $\phi_{\text {error.3 }}(m, n, t)$ denotes

$$
\frac{S(0, m-n, t)}{t} \mathrm{P} \int_{0}^{1 / 2} H \sum_{\substack{k=-\infty \\ k \neq-z / t}}^{\infty} \hat{f}\left(H\left(k+\frac{z-s}{t}\right)\right) \lambda(s) e\left(\frac{m+n}{N s t}\right) \mathscr{J}\left(\frac{4 \pi \sqrt{m n}}{N s t}\right) \frac{d s}{s} .
$$

Recalling its definition in 3.3 , in the above we have used the observation that in the case of $z \equiv 0(\bmod t)$ we simplify easily the exponential sum $\mathscr{S}_{0}(m, n, t)$ to the Kloosterman sum $S(0, m-n, t)$. We are going to give an upper bound for the two error terms just introduced above. Indeed one establishes that

$$
\phi_{\text {error.2 }}(m, n, t), \phi_{\text {error. } 3}(m, n, t) \ll t H\left(\frac{t}{H}\right)^{j},
$$

for any $j \geq 2$, which is the same upper bound as found earlier for $\phi_{\text {error.1 }}(m, n, t)$. Justifying both bounds can be done by a very similar argument and so we will only give more details for the slightly harder one, which is estimating $\phi_{\text {error.2 }}(m, n, t)$. We obtain immediately that the error term $\phi_{\text {error.2 }}(m, n, t)$ does not exceed

$$
H \sum_{\substack{z \bmod t \\ z \neq 0 \bmod t}} \int_{0}^{1 / 2} \sum_{k=-\infty}^{\infty}\left|\hat{f}\left(H\left(k+\frac{z-s}{t}\right)\right) \mathscr{J}\left(\frac{4 \pi \sqrt{m n}}{N s t}\right)\right| \frac{d s}{s}
$$

where we recall that from its definition we have that $\lambda(x) \ll 1$, for all $x \in \mathbb{R}_{\geq 0}$. Now using the upper bound for the Fourier transform $\hat{f}$, as stated earlier, and bounding the sum over the residue classes by $t$, we estimate the last expression by

$$
t H\left(\frac{t}{H}\right)^{j} \sum_{k=-\infty}^{\infty}\left(\frac{1}{|k|+1 / 2}\right)^{j} \int_{0}^{\infty}\left|\mathscr{J}\left(\frac{4 \pi \sqrt{m n}}{N s t}\right)\right| \frac{d s}{s},
$$

for any choice of $j \geq 2$. In the integral we can perform a change of variables $(4 \pi \sqrt{m n}) /(N s t) \mapsto 1 / y$, as a result of which the integral is seen to be independent from all $m, n, N$, $t$. From Lemma 7.5 we then know that $\mathscr{J}(1 / y) \in L^{1}\left(\mathbb{R}_{\geq 0}, d y / y\right)$, which immediately leads to the conclusion that

$$
\phi_{\text {error. } 2}(m, n, t) \ll t H\left(\frac{t}{H}\right)^{j},
$$


for any $j \geq 2$. Now what is left to do is removing the function $\lambda$ in the main term of the asymptotic expansion derived in 3.20 . To this end we may rewrite its main term as

$$
\frac{S(0, m-n, t)}{t} \mathrm{P} \int_{0}^{\infty} H \hat{f}\left(\frac{H s}{t}\right) e\left(\frac{m+n}{N s t}\right) \mathscr{J}\left(\frac{4 \pi \sqrt{m n}}{N s t}\right) \frac{d s}{s}+\phi_{\text {error. } 4}(m, n, t)
$$

where the error term $\phi_{\text {error. } 4}(m, n, t)$ represents the integral

$$
\frac{S(0, m-n, t)}{t} \mathrm{P} \int_{1 / 3}^{\infty} H \hat{f}\left(\frac{H s}{t}\right)(\lambda(s)-1) e\left(\frac{m+n}{N s t}\right) \mathscr{J}\left(\frac{4 \pi \sqrt{m n}}{N s t}\right) \frac{d s}{s} .
$$

We point out that, recalling the definition of $\lambda$, we avoid integrating the Fourier transform $\hat{f}$ over an interval close to zero. As explained earlier for small argument the bound given for $\hat{f}$ only tells us that it is bounded by a constant. We do not know that it is small here. However since the range of integration in the integral in $\phi_{\text {error. } 4}(m, n, t)$ is bounded below by $1 / 3$, we see that the latter error term is dominated by

$$
H \int_{1 / 3}^{\infty}\left(\frac{H s}{t}+1\right)^{-j}\left|\mathscr{J}\left(\frac{4 \pi \sqrt{m n}}{N s t}\right)\right| \frac{d s}{s},
$$

employing again the bound for the Fourier transform $\hat{f}$. We estimate this, first by dropping the one in the denominator, which we can do since $s$ is not too small, and then bound the resulting polynomial in $s$ by a constant depending on $j$. Now we can extend the range of integration to all of $\mathbb{R}_{>0}$, and proceed bounding the integral just as we did before, when we estimated (3.22). Thus after the appropriate change of variables and an application of Lemma 7.5 we arrive at

$$
\phi_{\text {error. } 4}(m, n, t) \ll t H\left(\frac{t}{H}\right)^{j} .
$$

Finally, before we can put everything together to conclude the theorem, we alter the main term in 3.23 . slightly. From the definition of the Fourier transform we have

$$
H \hat{f}\left(\frac{H s}{t}\right)=\int_{\mathbb{R}} f\left(\frac{y}{H}\right) e\left(\frac{y s}{t}\right) d y,
$$

by the change of variables $-H y \mapsto y$ and the assumption that $f$ is an even function. Now having inserting the latter integral for the multiple of the Fourier transform in the main term in 3.23), we interchange the order of integration of the $s$ integral and the one coming from $\hat{f}$. This is allowed by Fubini's Theorem since this double integral converges absolutely. An observation which is easily justified by pointing out that, after the appropriate change of variables, Lemma 7.5 ensures

$$
\int_{0}^{\infty}\left|\mathscr{J}\left(\frac{4 \pi \sqrt{m n}}{N s t}\right)\right| \frac{d s}{s} \ll 1,
$$

and that the cut-off function $f$ admits $\operatorname{supp}(f) \subseteq[-2,2]$. Hence the main term in $\sqrt{3.23}$ is then equal to

$$
\frac{S(0, m-n, t)}{t} \int_{\mathbb{R}} f\left(\frac{y}{H}\right) \mathrm{P}\left(\int_{0}^{\infty} e\left(\frac{y s}{t}+\frac{m+n}{N s t}\right) \mathscr{J}\left(\frac{4 \pi \sqrt{m n}}{N s t}\right) \frac{d s}{s}\right) d y
$$

The operator $\mathrm{P}$ acting on the $s$ integral is again an integral over a Hankel transform, which we evaluate explicitly using Lemma 7.6. Now, with the so rewritten main term in 3.23, we can combine this asymptotic with (3.20) and via (3.19) with (3.18) conclude that

$$
\widetilde{\phi_{f}^{0}}(m, n, t)=\phi_{f}^{0}(m, n, t)+\phi_{\text {error. } 5}(m, n, t),
$$


where $\phi_{f}^{0}(m, n, t)$ is the desired second main term, as claimed in Theorem 3.2 The error term in the latter is combined

$$
\phi_{\text {error.5 }}(m, n, t):=\sum_{i=2}^{4} \phi_{\text {error.i }}(m, n, t) \ll t H\left(\frac{t}{H}\right)^{j},
$$

for any $j \geq 2$. The last estimation is the result of invoking (3.21) and 3.24). Employing the asymptotic just found in 3.25 and that we have shown previously $\widetilde{\phi_{f}}(m, n, t)=\phi_{f}(m, n, t)$, we conclude via 3.16 and 3.10 ) that

$$
\phi(m, n, t)=\phi_{f}(m, n, t)-\phi_{f}^{0}(m, n, t)+\phi_{\text {error }}(m, n, t),
$$

where the error, for any $j \geq 2$, is estimated through

$$
\phi_{\text {error }}(m, n, t):=\phi_{\text {error. } 1}(m, n, t)-\phi_{\text {error.5 }}(m, n, t) \ll t H\left(\frac{t}{H}\right)^{j},
$$

as a result of the bounds found in 3.14 and 3.26. This almost finishes our considerations regarding Theorem 3.2. En route to giving a complete argument supporting the claims of the theorem, the last piece is to prove Lemma 3.3 , which we are going to do now.

\subsection{Controlling an oscillatory integral}

In this section we will give a proof for Lemma 3.3 This result essentially tells us, that, in the absence of stationary phase of the phase function of the exponential in the integrand, the integral in (3.12) is very small as soon as we have $|h|>t$ and is bounded by a constant otherwise. It is in the same spirit as the bound given for the oscillatory integral at the bottom of [15, p.546] and we employ essentially the same strategy as in [15], which is repeated integration by parts, to obtain our result.

Proof of Lemma 3.3 We start our argument by simplifying the notation in this section a little and thus denote in the following

$$
k=k(m, n, N, t):=4 \pi \sqrt{m n} /(N t) .
$$

Now we assume that $|h| \leq t$. Then after performing the change of variables $k / s \mapsto 1 / s$ in the $\mathrm{s}$ integral in 3.12, with Lemma 7.5 we obtain directly that

$$
\int_{0}^{\infty} \varrho(s) e\left(\frac{h s}{t}+\frac{m+n}{N s t}\right) \mathscr{J}\left(\frac{k}{s}\right) \frac{d s}{s} \ll 1 .
$$

We are left to consider the case of $|h|>t$ and show that here the integral in 3.12 is indeed small. In order to make progress we write the integral in question as a sum of integrals that have a shorter range of integration and in which the integrand has compact support in each of them. We follow this plan by introducing a partition of unity of $\mathbb{R}_{>0}$, being the family of smooth functions $\mathrm{p}_{l}: \mathbb{R}_{>0} \rightarrow \mathbb{R}$, that have $\operatorname{supp}\left(\mathrm{p}_{l}\right) \subseteq\left[3^{l-1}, 3^{l+1}\right]$, satisfy

$$
\sum_{l \in \mathbb{Z}} \mathrm{p}_{l}(x)=1, \text { for every } x \in \mathbb{R}_{>0},
$$

and admit the bounds $\mathrm{p}_{l}^{(j)}(x) \ll 3^{-l j}$, for any choice of $l \in \mathbb{Z}$ and $j \geq 0$. We swap the order of integration in the double integral (3.12), recalling the definition of $\mathscr{J}$ from 2.7). This is allowed by Fubini's Theorem, since the double integral converges absolutely, which is seen quickly by performing the change of variables 
$k / s \mapsto 1 / s$ and then applying Lemma 7.5 Thus bringing the partition of unity into the picture, we use it to dissect the $s$-summation into intervals of length $8 \cdot 3^{l-1}$, and find that the double integral just described equals in fact

$$
\frac{2 \mathrm{i}}{\pi} \int_{\mathbb{R}} \frac{w h(w)}{\cosh (\pi w)} \sum_{l \in \mathbb{Z}} \mathfrak{P}(w ; l) d w
$$

where we define

$$
\mathfrak{P}(w ; l):=\int_{3^{l-1}}^{3^{l+1}} \mathrm{p}_{l}(s) \varrho(s) e\left(\frac{h s}{t}+\frac{m+n}{N s t}\right) J_{2 \mathrm{i} w}\left(\frac{k}{s}\right) \frac{d s}{s} .
$$

We have to consider two scenarios. The function $k$ may exceed $1 / 3$ or not. In the argument to follow we will only present estimates for the case of $k \geq 1 / 3$, since if this is not the case the desired bounds actually follow by the same ansatz as chosen for the situation when we encounter in the integral $\sqrt{3.29}$ that $s \geq k \geq 1 / 3$. Thus we assume that $k \geq 1 / 3$ and then split the $l$ sum in 3.28 according to $k$ to see that it equals

$$
\sum_{-2 \leq l<\left\lceil\frac{\log k}{\log 3}\right\rceil+1} \mathfrak{P}(w ; l)+\sum_{l \geq\left\lceil\frac{\log k}{\log 3}\right\rceil+1} \mathfrak{P}(w ; l),
$$

where the first (finite) sum is relatively short with about $\log k$ terms and the second (infinite) sum satisfies that in in the integral $\mathfrak{P}(w ; l)$ we always have $s \geq k$. We deal with the short sum first. In this case almost in the whole range of integration of $(3.29)$ we encounter $s \leq k$, which means that the argument of the $J$-Bessel function in the integrand of this integral is bigger than one, when this happens. Hence the function oscillates there and estimating $\mathfrak{P}(w ; l)$ by integrating by parts multiple times we can exploit that. Employing Lemma 7.7 we may extract and identify this oscillation by replacing the $J$-Bessel function by a sum of products of functions, of which one oscillates and the other one and its derivatives are easy to estimate. Thus in order to control the integral $\mathfrak{P}(w ; l)$, we see that it suffices to control the two integrals

$$
\frac{1}{(2 \pi k)^{1 / 2}} \sum_{ \pm} \int_{3^{l-1}}^{3^{l+1}} \frac{\mathrm{p}_{l}(s) \varrho(s) \Omega_{ \pm}(2 w \mathrm{i}, k /(2 \pi s))}{s^{1 / 2}} e\left(\frac{h s}{t}+\frac{m+n \pm 2 \sqrt{m n}}{N s t}\right) d s .
$$

We are going to integrate by parts multiple times and bound them by means of [1, Lemma 8.1], which gives an estimate for certain oscillatory integrals. Choosing a similar notation as in the lemma we set

$$
\omega_{ \pm}(s):=\frac{\mathrm{p}_{l}(s) \varrho(s) \Omega_{ \pm}(2 w \mathrm{i}, k /(2 \pi s))}{s^{1 / 2}} \text { and } \quad \mathscr{H}_{ \pm}(s):=2 \pi\left(\frac{h s}{t}+\frac{m+n \pm 2 \sqrt{m n}}{N s t}\right),
$$

the latter being the phase function of the exponential. By assumption we demand that

$$
|h| \geq 81(m+n) / N,
$$

which allows us to deduce easily the necessary bounds for the derivatives of the phase function of the exponential given by

$$
\left|\mathscr{H}_{ \pm}^{\prime}(s)\right| \geq|h| / t \text { and } \mathscr{H}_{ \pm}^{(j)}(s) \ll|h| / t \text {, for all } j \geq 1 .
$$

In particular the lower bound for the first derivative tells us that we do not experience any stationary phase in the integrals 3.31. We have to bound the remaining function and its derivatives in the integrand of 3.31. Indeed we obtain for them quickly that

$$
\omega_{ \pm}^{(j)}(s) \ll e^{3 \pi|w|}, \text { for all } j \geq 0
$$


where we use (7.23) to estimate $\Omega_{ \pm}$and its derivatives and the fact that $s \geq 1 / 3$. Furthermore be observe that $\operatorname{supp}\left(\omega_{ \pm}\right) \subseteq \operatorname{supp}\left(\mathrm{p}_{l}\right) \cap(1 / 3, \infty)$. Thus in light of (3.33) and 3.34) we have from [1, Lemma 8.1] that (3.31) is dominated by

$$
\frac{3^{l} e^{3 \pi|w|}}{k^{1 / 2}}\left(\frac{|h|}{t}\right)^{-j}, \text { for any } j \geq 1 .
$$

Thus with this upper bound for $\mathfrak{P}(w ; l)$ in the case of $l \ll \log k$ in place, we bound this part of 3.28, recalling the finite sum in 3.30, by

$$
\begin{aligned}
& \frac{2 \mathrm{i}}{\pi} \int_{\mathbb{R}} \frac{w h(w)}{\cosh (\pi w)} \sum_{-2 \leq l<\left\lceil\frac{\log k}{\log 3}\right\rceil+1} \mathfrak{P}(w ; l) d w \ll\left(\frac{|h|}{t}\right)^{-(j+1)} \frac{1}{k^{1 / 2}} \sum_{-2 \leq l<\left\lceil\frac{\log k}{\log 3}\right\rceil+1} 3^{l} \\
& \ll\left(\frac{|h|}{t}\right)^{-(j+1)} k^{1 / 2+\varepsilon} \ll\left(\frac{|h|}{t}\right)^{-j},
\end{aligned}
$$

adjusting the number of times $j$ we integrate by parts appropriately, since assuming 3.32 ensures $k \ll$ $|h| / t$. We are left with handling the part of the $l$-summation in 3.28$)$, for which all integrals $\mathfrak{P}(w ; l)$ considered therein have a range of integration, that is bounded below by $k$ (cp. the infinite sum in (3.30). This results in $s>k$, meaning that the argument of the $J$-Bessel function is always less than one, i.e. the Bessel function does not oscillate here. Following the same strategy as before, trying to obtain a good bound by integrating by parts repeatedly, any saving has to come solely from the oscillation of the exponential contained in the integrand of 3.29). We begin by shifting the contour in the $w$ integral in 3.29 to $\Im(w)=$ $-\varepsilon / 2$, for a $\varepsilon>0$ small enough so that we avoid all poles. That leaves us with estimating the integral

$$
\int_{3^{l-1}}^{3^{l+1}} \frac{\mathrm{p}_{l}(s) \varrho(s) J_{2 \mathrm{i} w+\varepsilon}(k / s)}{s} e\left(\frac{h s}{t}+\frac{m+n}{N s t}\right) d s .
$$

Similarly to our previous considerations for the bounded $l$ sum we aim to use [1, Lemma 8.1]. Hence as before we define in a similar fashion to the notation in this lemma

$$
\omega_{1}(s):=\frac{\mathrm{p}_{l}(s) \varrho(s) J_{2 \mathrm{i} w+\varepsilon}(k / s)}{s} \text { and } \mathscr{H}_{1}(s):=2 \pi\left(\frac{h s}{t}+\frac{m+n}{N s t}\right) .
$$

For the derivatives of the phase function of the exponential we easily see, under the assumption of [3.32, the estimates

$$
\left|\mathscr{H}_{1}^{\prime}(s)\right| \geq|h| / t \text { and } \mathscr{H}_{1}^{(j)}(s) \ll(|h| / t) \cdot 3^{-l(j+1)} \text {, for all } j \geq 2,
$$

where again the lower bound for the first derivative guarantees that the integral we try to estimate does not have stationary phase. To obtain the bound we use that in the relevant range of integration we have $s \asymp 3^{l}$ and $s \geq 1 / 3$. For the remaining integrand we obtain the bounds

$$
\omega_{1}^{(j)}(s) \ll e^{2 \pi|w|} k^{\varepsilon} \cdot 3^{-l(1+\varepsilon)}, \text { for all } j \geq 0,
$$

again noticing that $s \asymp 3^{l}$ and where we combine Lemmata 7.1 and 7.2 to bound the $J$-Bessel function and its derivatives by

$$
\frac{\partial^{j}}{\partial s^{j}} J_{2 \mathrm{i} w+\varepsilon}(k / s) \ll e^{\pi|w|}\left(1+|w|^{j}\right)(k / s)^{\varepsilon}, \text { for all } j \geq 0,
$$


in light of $s>k \geq 1 / 3$. Furthermore we have $\operatorname{supp}\left(\omega_{ \pm}\right) \subseteq \operatorname{supp}\left(\mathrm{p}_{l}\right)$. Thus with the bounds from 3.37) and (3.38) we now conclude from [1, Lemma 8.1] that the integral (3.36) does not exceed

$$
\frac{e^{2 \pi|w|}}{3^{l \varepsilon}}\left(\frac{|h|}{t}\right)^{-j}, \text { for any } j \geq 1
$$

Thus we now obtain with this bound for the unbounded part of the $l$-summation in 3.28 (

$$
\begin{aligned}
& \frac{2 \mathrm{i}}{\pi} \int_{\mathbb{R}} \frac{(w-\mathrm{i} \varepsilon / 2) h(w-\mathrm{i} \varepsilon / 2)}{\cosh (\pi(w-\mathrm{i} \varepsilon / 2))} \sum_{l \geq\lceil\log k\rceil} \int_{3^{l-1}}^{\left.3^{l+1}\right\rceil} \frac{\mathrm{p}_{l}(s) \varrho(s) J_{2 \mathrm{i} w+\varepsilon}(k / s)}{s} e\left(\frac{h s}{t}+\frac{m+n}{N s t}\right) d s d w \\
& \ll\left(\frac{|h|}{t}\right)^{-j} \sum_{l=1}^{\infty} \frac{1}{3^{l \varepsilon}} \ll\left(\frac{|h|}{t}\right)^{-j},
\end{aligned}
$$

with the implied constant depending on the Schwartz function $h$. We combine the bounds of (3.35) and 3.39 to conclude via 3.28 that for $|h|>t$ we have

$$
\int_{0}^{\infty} \varrho(s) e\left(\frac{h s}{t}+\frac{m+n}{N s t}\right) \mathscr{J}\left(\frac{k}{s}\right) \frac{d s}{s} \ll\left(\frac{|h|}{t}\right)^{-j}, \text { for any } j \geq 1 .
$$

Finally upon merging the bounds given in 3.27) and 3.40) we conclude the lemma.

Remark 3.4. We conclude this section with a remark. Previously in the beginning of Subsection 3.2.1 we have introduced the function $\varrho$. In the sum (3.7), that first features this function, inserting it at first seems obsolete. However after having applied Poisson summation to it, the resulting expression (3.8) contains an integral, with a range of integration starting on the lower end slightly away from zero. We recall here that $\operatorname{supp}(\varrho) \subseteq[1 / 3, \infty)$. Without having at our disposal that in the integral in $(3.12)$, we may invoke the lower bound $s \geq 1 / 3$ for the integration variable $s$, many essential estimates in this section would not be possible. Hence introducing the function @ is de rigueur in order to establish Lemma 3.3. This described tool for getting rid of a short interval next to zero in the range of integration in the mentioned integral, is just as employed in [15. Chapter 3: An asymptotic trace formula]. 



\section{Chapter 4}

\section{An asymptotic for quadratic forms for the full spectrum}

\subsection{The Bruggeman-Kuznetsov formula for quadratic forms}

In this chapter we are going to develop results in the context of quadratic forms. These are based and derived from the results given in the previous Chapter 3 The theorems therein deal with the case of some fixed $m, n \in \mathbb{N}$. In this chapter we are going to average $m, n$ over the interval $(M, 2 M]$, summing against complex numbers. To make this plan more concrete we introduce some notation. The space of complex vectors with support in the interval $(M, 2 M]$, for some $M \geq 1$, be given by

$$
\mathbb{C}_{M}^{\infty}:=\left\{\alpha=\left(a_{m}\right)_{m \in \mathbb{N}} \in \mathbb{C}^{\infty} \mid \operatorname{supp}(\alpha) \subseteq(M, 2 M]\right\},
$$

for which we may now consider functions mapping this space into the complex numbers. With this setup in place we may now convert the Bruggeman-Kuznetsov formula into the lineup of quadratic forms. To this end we recall the version from 2.9 in Theorem 2.3. multiply both side with $\bar{a}_{m} a_{n}$, for any choice of $a_{h} \in \mathbb{C}$, and sum for both summation indices over the interval $(M, 2 M]$. We describe the spectral side of the resulting formula in terms of the quadratic forms

$$
\xi(\alpha):=\xi^{\operatorname{disc}}(\alpha)+\xi^{\operatorname{cont}}(\alpha),
$$

where

$$
\xi^{\operatorname{disc}}(\alpha):=\frac{2}{\varphi(N)} \sum_{\chi \bmod N}^{+} \sum_{u \in \mathcal{U}_{\chi}^{\text {Maaß }}}\left|\sum_{M<m \leq 2 M} a_{m} \rho_{u}(m)\right|^{2} \frac{h\left(t_{u}\right)}{\cosh \left(\pi t_{u}\right)}
$$

and

$$
\xi^{\mathrm{cont}}(\alpha):=\frac{1}{2 \pi \varphi(N)} \sum_{\chi \bmod N}^{+} \sum_{\mathfrak{a}} \int_{\mathbb{R}}\left|\sum_{M<m \leq 2 M} a_{m} \tau_{\mathfrak{a}, \chi}(m, w)\right|^{2} \frac{h(w)}{\cosh (\pi w)} d w .
$$

The arithmetic side features two terms. Reminiscent of the diagonal term we see the 2-norm of the vector $\alpha$ multiplied by a constant depending on the function $h$, and the remaining terms are given by

$$
\Phi(\alpha):=\sum_{M<m, n \leq 2 M} \bar{a}_{m} a_{n} \phi(m, n)
$$

with $\phi(m, n)$ as defined in 2.10. We summarise the obtained formula in the following lemma. 
Lemma 4.1 (Bruggeman-Kuznetsov formula for quadratic forms). For any $\alpha \in \mathbb{C}_{M}^{\infty}$ and with the notation and assumptions of Theorem 2.3 we have

$$
\xi(\alpha)=\|\alpha\|^{2} \int_{\mathbb{R}} h(w) d_{\mathrm{spec}} w+\Phi(\alpha) .
$$

Again the Schwartz function $h$ is assumed to admit the properties specified in Section 2.4

The goal of this chapter is to derive an asymptotic formula for the quadratic form $\xi: \mathbb{C}_{M}^{\infty} \rightarrow \mathbb{C}$, with an error term proportional to the 2-norm of the vector considered. The point of origin for this expansion will be the Bruggeman-Kuznetsov formula for quadratic forms in the version which we just introduced with Lemma 4.1. We give a precise formulation of the asymptotic formula for $\xi(\alpha)$, that we are going to establish in this chapter, in the next section.

\subsection{The main theorem}

We are going to state here the main result of this chapter. It is an asymptotic expansion of the quadratic form $\xi(\alpha)$. The main term resembles certain parts of $\Phi(\alpha)$ and the error is obtained by estimating the remaining bits thereof. It is proportional to the 2-norm of the vector $\alpha$. The precise statement is the following.

Theorem 4.2. We consider any smooth even cut-off function $f: \mathbb{R} \rightarrow \mathbb{R}_{\geq 0}$, which equals one on $[-1,1]$ and satisfies $\operatorname{supp}(f) \subseteq[-2,2]$. For an odd prime level $N$ we choose $H=327 T^{1+\delta}$ and $T=M / N>1$. Then, for any $\alpha \in \mathbb{C}_{M}^{\infty}$, we find the asymptotic formula

$$
\xi(\alpha)=\mathscr{M}(\alpha)+\mathcal{O}\left(\|\alpha\|^{2}\left(1+\frac{M}{N^{2}}\right) M^{\varepsilon}\right)
$$

where the main term $\mathscr{M}(\alpha)$ is given by

$$
\frac{1}{N} \sum_{\substack{1 \leq t \leq T \\(t, N)=1}} \frac{1}{t^{2}} \sum_{0 \leq|h| \leq 2 H} f\left(\frac{h}{H}\right) \sum_{M<m, n \leq 2 M} \bar{a}_{m} a_{n} S(h \bar{N}, m, t) S(h \bar{N}, n, t) \mathscr{I}\left(\frac{m}{N t}, \frac{n}{N t}, \frac{h}{t}\right) .
$$

The function $\mathscr{I}$, for the first two variables being $m / N t$ and $n / N t$ respectively, is given as follows. If the third variable $h / t$ is positive, it is the integral

$$
-\mathrm{i} \int_{\mathbb{R}} \frac{4 \pi^{2} h(x)}{\sinh ^{2}(\pi x)} J_{2 \mathrm{i} x}\left(\frac{4 \pi}{t} \sqrt{\frac{h m}{N}}\right) \Im\left(J_{2 \mathrm{i} x}\left(\frac{4 \pi}{t} \sqrt{\frac{h n}{N}}\right)\right) d_{\text {spec }} x
$$

Provided the third variable $h / t$ takes negative values it is

$$
\mathrm{i} \int_{\mathbb{R}} \frac{8 \pi h(x)}{\tanh (\pi x)} Y_{2 \mathrm{i} x}\left(\frac{4 \pi}{t} \sqrt{\frac{|h| \max \{m, n\}}{N}}\right) K_{2 \mathrm{i} x}\left(\frac{4 \pi}{t} \sqrt{\frac{|h| \min \{m, n\}}{N}}\right) d_{\mathrm{spec}} x
$$

and if the third variable is equal to zero it becomes

$$
\frac{2}{\pi} \int_{\mathbb{R}} h(x)\left(\frac{\sqrt{m n}}{\max \{m, n\}}\right)^{2 \mathrm{i} x} d x .
$$

As before $J, Y$ and $K$ denote the corresponding Bessel functions. 


\subsection{Proof of the main theorem}

In this section we will now give a full proof of Theorem 4.2. The structure and layout of our argument is similar to the one used to prove [15. Theorem 10.1] and we follow their ansatz and strategy, as well as some individual steps, in [15] adapting it to our situation. The argument we are going to present is based on several intermediate results, that will be just stated here and only justified at a later stage in this chapter.

Proof. The goal here is to find an asymptotic expansion of $\Phi(\alpha)$. Having found one via Lemma 4.1 we may then directly translated it into one for $\xi(\alpha)$. We point out again that we work under the assumption that $N$ is an odd prime number. Then by recalling the formula in 3.5 we may write

$$
\phi(m, n)=\phi^{\text {thin }}(m, n)+\sum_{\substack{t=1 \\(t, N)=1}}^{\infty} \frac{\phi(m, n, t)}{N t},
$$

with $\phi(m, n, t)$ as given in 3.6 and where in light of 3.4 we define

$$
\phi^{\text {thin }}(m, n):=\mathrm{P} \sum_{y=1}^{\infty} \sum_{t=1}^{\infty} \frac{\mathscr{S}_{N y}(m, n, N t)}{N^{2} y t} e\left(\frac{m+n}{N^{2} y t}\right) \mathscr{J}\left(\frac{4 \pi \sqrt{m n}}{N^{2} y t}\right) .
$$

The split into two terms in (4.6) allows us to thin out in some sense the term $\Phi(\alpha)$, estimating its component for which in the above $t$-summation we have $t \equiv 0(\bmod N)$, collected in the term $\phi^{\text {thin }}(m, n)$, and leaving us to consider the terms for which $(t, N)=1$. Indeed we can show the following.

Lemma 4.3. For any $\alpha \in \mathbb{C}_{M}^{\infty}$ we have

$$
\Phi^{\text {thin }}(\alpha) \ll\|\alpha\|^{2} \frac{M^{1+\varepsilon}}{N^{2}},
$$

where we define coherent with the previous notation in this chapter

$$
\Phi^{\text {thin }}(\alpha):=\sum_{M<m, n \leq 2 M} \bar{a}_{m} a_{n} \phi^{\text {thin }}(m, n) .
$$

As a consequence of this lemma we obtain, with the split in 4.6 in mind, the preliminary expansion of $\Phi(\alpha)$ given by

$$
\Phi(\alpha)=\Phi_{t \leq T}(\alpha)+\Phi_{t>T}(\alpha)+\mathcal{O}\left(\|\alpha\|^{2} \frac{M^{1+\varepsilon}}{N^{2}}\right) .
$$

In the asymptotic formula just stated we have separated the main term according to the size of the summation index $t$, relative to some cut-off parameter $T$, which we will make more precise in due course. In particular in this formula we define the terms $\Phi_{t \leq T}(\alpha)$ and $\Phi_{t>T}(\alpha)$ respectively by

$$
\sum_{M<m, n \leq 2 M} \bar{a}_{m} a_{n} \sum_{\substack{t \leq T \\(t, N)=1}} \frac{\phi(m, n, t)}{N t} \text { and } \sum_{M<m, n \leq 2 M} \bar{a}_{m} a_{n} \sum_{\substack{t>T \\(t, N)=1}} \frac{\phi(m, n, t)}{N t} .
$$

The characteristic size of the error term in the asymptotic given in Theorem 4.2 is due to the bound we obtain for $\Phi_{t>T}(\alpha)$. The following result makes this precise.

Lemma 4.4. Let $T \geq M / N>1$. Then for any $\alpha \in \mathbb{C}_{M}^{\infty}$ we find the bound

$$
\Phi_{t>T}(\alpha) \ll\|\alpha\|^{2}\left(1+\frac{M}{N^{2}}\right) .
$$


Going forward we will from now on choose in fact $T=M / N$. Thus with Lemma 4.4 the asymptotic formula from 4.8 becomes

$$
\Phi(\alpha)=\Phi_{t \leq T}(\alpha)+\mathcal{O}\left(\|\alpha\|^{2}\left(1+\frac{M}{N^{2}}\right) M^{\varepsilon}\right),
$$

for $T=M / N$. As stated before, we already see therein an error term of the size claimed in Theorem 4.2 . To establish the statement of the main result of this chapter, we are left to transform the main term $\Phi_{t \leq T}(\alpha)$ in 4.10 into the desired form. We do just that by using Theorem 3.2 to write $\phi(m, n, t)$ as a sum of three terms and via its definition in 4.9 we therefore arrive directly at

$$
\Phi_{t \leq T}(\alpha)=\mathscr{M}(\alpha)-\mathscr{M}^{0}(\alpha)+\Phi_{\text {error }}(\alpha),
$$

where the first main term $\mathscr{M}(\alpha)$ is as in 4.5 , upon recalling that the condition $(t, N)=1$ allows us to employ [15. Corollary 3.1] to simplify the sum over Kloosterman sums in the definition of $\phi_{f}(m, n, t)$. The remaining terms of the asymptotic formula are given by

$$
\mathscr{M}^{0}(\alpha):=\frac{1}{N} \sum_{\substack{t \leq T \\(t, N)=1}} \frac{1}{t^{2}} \sum_{M<m, n \leq 2 M} \bar{a}_{m} a_{n} S(0, m-n, t) \int_{\mathbb{R}} f\left(\frac{y}{H}\right) \mathscr{I}\left(\frac{m}{N t}, \frac{n}{N t}, \frac{y}{t}\right) d y
$$

and

$$
\Phi_{\text {error }}(\alpha):=\sum_{M<m, n \leq 2 M} \bar{a}_{m} a_{n} \sum_{\substack{t \leq T \\(t, N)=1}} \frac{\phi_{\text {error }}(m, n, t)}{N t} .
$$

We have used Theorem 3.2, which means we have to meet its requirements under which it is valid. In particular this means we have to use a cut-off parameter

$$
H \geq 2 t+81(m+n) / N .
$$

By the restriction on the summation indices $m, n$ and $t$ in $\Phi_{t \leq T}(\alpha)$ and with $T=M / N$ upon choosing

$$
H=327 T^{1+\delta}
$$

the condition in 4.13 is always satisfied and thus so is the application of Theorem 3.2. From this result, which provides an upper bound for the error $\phi_{\text {error }}(m, n, t)$, we then quickly infer the estimate

$$
\Phi_{\text {error }}(\alpha) \ll\|\alpha\|^{2},
$$

using the Cauchy Schwarz inequality and choosing $j$ large enough. What is left is to determine the maximum size of the second main term $\mathscr{M}^{0}(\alpha)$ in 4.11 . A suitable upper bound is stated in the following result.

Lemma 4.5. Let $H=327 T^{1+\delta}$ with $T=M / N>1$. Then again for every $\alpha \in \mathbb{C}_{M}^{\infty}$ we bound the term

$$
\mathscr{M}^{0}(\alpha) \ll\|\alpha\|^{2}\left(1+\frac{M}{N^{2}}\right) M^{\varepsilon} .
$$

Now we are very close to concluding Theorem 4.2. With the last lemma and the bound in 4.14) in mind, we combine the two expressions in (4.10) and 4.11) to obtain

$$
\Phi(\alpha)=\mathscr{M}(\alpha)+\mathcal{O}\left(\|\alpha\|^{2}\left(1+\frac{M}{N^{2}}\right) M^{\varepsilon}\right) .
$$

Indeed from the Bruggeman-Kuznetsov formula for quadratic forms, as stated in Lemma 4.1, we finally derive the statement of Theorem 4.2, noticing the diagonal term in this Bruggeman-Kuznetsov formula is in fact absorbed into the error term of the asymptotic just established for $\Phi(\alpha)$. 
The above proof relies on the three Lemmata 4.3, 4.4 and 4.5, which we still have to justify. A detailed presentation of their proofs is going to be the subject of the remaining sections of this chapter.

\subsection{Thinning out the main term}

In this section we will show that it is enough in Section 4.3 to work with a thinned out version of the term $\Phi(\alpha)$. Concretely this means we establish Lemma 4.3 , which provides the bound

$$
\Phi^{\text {thin }}(\alpha) \ll\|\alpha\|^{2} \frac{M^{1+\varepsilon}}{N^{2}},
$$

for any $\alpha \in \mathbb{C}_{M}^{\infty}$. Given the error term in Theorem 4.2 that is of exceptable size for us, the latter estimate is consumed thereof. Thus in light of of the split in (4.6) and recalling the definition

$$
\Phi(\alpha)=\sum_{M<m, n \leq 2 M} \bar{a}_{m} a_{n} \phi(m, n),
$$

it suffices to consider only those terms of the $t$-summation, where its index is coprime with the prime level $N$. Being left to consider this thinned out version of $\Phi(\alpha)$, we see that via 4.10 and (4.11) this leads us to consider a thinned out version of the main term $\mathscr{M}(\alpha)$, as featured in Theorem 4.2, too. The proof we are going to present in support of Lemma 4.3 in this section follows the strategy and outline of the argument proving [15, Lemma 10.1 (statement and proof on p. 557)].

Proof. In the following we will show the estimate for $\Phi^{\text {thin }}(\alpha)$ as claimed in Lemma 4.3 We recall the definition of $\phi^{\text {thin }}(m, n)$ in 4.7$)$ and by the definition of the operator P therein we have $\mathrm{P}(g)=g+\bar{g} \ll|g|$. Hence in order to be able to control the term $\Phi^{\text {thin }}(\alpha)$, it is clearly enough to bound only the sum

$$
\sum_{y, t=1}^{\infty} \frac{1}{N^{2} y t} \sum_{\substack{x \bmod N t \\(x(1+N y x), N t)=1}} \sum_{M<m, n \leq 2 M} \bar{a}_{m} a_{n} e\left(\frac{\bar{x} m-n \overline{(x+N y)}}{N t}\right) e\left(\frac{m+n}{N^{2} y t}\right) \mathscr{J}\left(\frac{4 \pi \sqrt{m n}}{N^{2} y t}\right),
$$

where we exchanged $\mathscr{S}_{N y}(m, n, N t)$ with its definition as a sum given in 3.3$)$. Essentially we obtain good bounds for $\Phi^{\text {thin }}(m, n)$ by shifting the contour in $\mathscr{J}$ conveniently and exploiting oscillation that comes form the exponential functions we sum over in $\mathscr{S}_{N y}(m, n, N t)$. The latter is going to be facilitated via the use of large sieve inequalities for additive characters, which we will explain in full detail in due course. For now we need to set the stage, so that these inequalities can be put in play. Hence as a first step we need to separate variables in the $J$-Bessel function in the integral transform $\mathscr{J}$. We will use different approaches depending on whether its argument exceeds one or is uniformly bounded by a constant. To distinguish which case we are dealing with, we split the summation in 4.15 into

$$
\sum_{y=1}^{\infty} \sum_{1 \leq t<\frac{4 \pi M}{N^{2} y}} \cdots+\sum_{y=1}^{\infty} \sum_{\frac{4 \pi M}{N^{2} y} \leq t} \cdots
$$

where we interpret the $t$-summation as zero, if it is empty. We begin to deal with the summation in which $t$ has a lower bound depending on $y$. In this case the argument of the Bessel function is bounded. Therefore we may use its power series representation to separate variables, without losing control over the size of the function. Before we continue in this direction we further need to assess which terms is the $t$-summation 
exceed $M / N$ and the ones that don't. To make this distinction we first split the $y$-summation and obtain

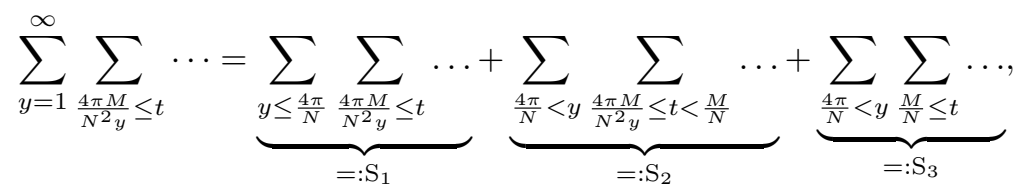

where again we interpret $\mathrm{S}_{1}$ and $\mathrm{S}_{2}$ as zero, should the summation be empty. In $\mathrm{S}_{1}$ we have $M / N \leq$ $4 \pi M / N^{2} y \leq t$, for all $y$ considered here. When we consider the sums $\mathrm{S}_{1}, \mathrm{~S}_{3}$ we shift the contour in $\mathscr{J}$ to $\Im(w)=-(\varepsilon+1) / 2$, and for $\mathrm{S}_{2}$ to $\Im(w)=-\varepsilon / 2$. Now for all three sums the following considerations are in fact the same. From [10, 8.402] for $x \in \mathbb{R}_{>0}$ we have

$$
J_{\nu}(x)=\sum_{k=0}^{\infty} \frac{(-1)^{k}}{k ! \Gamma(\nu+k+1)}\left(\frac{x}{2}\right)^{2 k+\nu},
$$

which we rewrite the $J$-Bessel function in 4.15 with. Therefore we observe that every sum in 4.17 is bounded by

$$
\int_{\mathbb{R}}\left|\frac{h\left(w-\mathrm{i} c_{\varepsilon} / 2\right)\left(w-\mathrm{i} c_{\varepsilon} / 2\right)}{\cosh \left(\pi\left(w-\mathrm{i} c_{\varepsilon} / 2\right)\right) \Gamma\left(2 \mathrm{i} w+c_{\varepsilon}+1\right)}\right| \sum_{y, t} \sum_{k=0}^{\infty} \frac{\left|\mathcal{G}_{k, y, t}(\alpha ; w)\right|}{4^{k}(k !)^{2} N^{2} y t}\left(\frac{1}{N^{2} y t}\right)^{2 k+c_{\varepsilon}} d w
$$

with $c_{\varepsilon}=\varepsilon+1$ for sums $\mathrm{S}_{1}$ and $\mathrm{S}_{3}$, and $c_{\varepsilon}=\varepsilon$ for $\mathrm{S}_{2}$ and where the $y, t$-summation run according to the case we consider; via the Cauchy Schwarz inequality, and afterwards enlarging by positivity the corresponding sums to run over all residue classes, we have that $\mathcal{G}_{k, y, t}(\alpha ; w)$ is dominated by

$$
\left(\sum_{x \bmod N t}\left|\sum_{M<m \leq 2 M} \widetilde{A}_{m}^{k, y, t}(w) e\left(\frac{x m}{N t}\right)\right|^{2}\right)^{1 / 2}\left(\sum_{x \bmod N t}\left|\sum_{M<n \leq 2 M} A_{n}^{k, y, t}(w) e\left(\frac{x n}{N t}\right)\right|^{2}\right)^{1 / 2}
$$

where

$$
\widetilde{A}_{m}^{k, y, t}(w):=\bar{a}_{m} m^{k+\mathrm{i} w+c_{\varepsilon} / 2} e\left(m / N^{2} y t\right) \quad \text { and } \quad A_{n}^{k, y, t}(w):=a_{n} n^{k+\mathrm{i} w+c_{\varepsilon} / 2} e\left(n / N^{2} y t\right) .
$$

Each factor in the bound for $\mathcal{G}_{k, y, t}(\alpha ; w)$ is now estimated with the large sieve inequality for additive characters as given in [14, Theorem 7.7]. We apply the latter large sieve inequality for the family of harmonics $\{e(x m /(N t))\}$, for points $x /(N t)$ in the unit interval, where $x$ is a residue class of modulus $N t$. Any such two distinct points are easily seen to be at least $(N t)^{-1}$ apart. Hence with [14, Theorem 7.7] we obtain

$$
\mathcal{G}_{k, y, t}(\alpha ; w) \ll\|\alpha\|^{2}(N t+M) M^{2 k+c_{\varepsilon}} .
$$

Using the latter bound we find that 4.18 does not exceed

$$
\int_{\mathbb{R}}\left|\frac{h\left(w-\mathrm{i} c_{\varepsilon} / 2\right)\left(w-\mathrm{i} c_{\varepsilon} / 2\right)}{\cosh \left(\pi\left(w-\mathrm{i} c_{\varepsilon} / 2\right)\right) \Gamma\left(2 \mathrm{i} w+c_{\varepsilon}+1\right)}\right| d w \sum_{y, t} \frac{N t+M}{N^{2} y t}\left(\frac{M}{N^{2} y t}\right)^{c_{\varepsilon}}\|\alpha\|^{2},
$$

where we employ the fact that the argument of the Bessel function is uniformly bounded by a constant, for the ranges of summation of $y$ and $t$ which we treat here. Stirling approximation provides the upper bound

$$
\Gamma\left(2 \mathrm{i} w+c_{\varepsilon}+1\right)^{-1} \ll e^{\pi|w|}(1+|w|)^{-c_{\varepsilon}-1 / 2} .
$$


Thus the integral in 4.19 converges, since $h$ is a Schwartz function satisfying appropriate decay properties as specified in Section 2.4, and we conclude the following bounds

$$
\mathrm{S}_{1}, \mathrm{~S}_{3} \ll\|\alpha\|^{2} \sum_{y=1}^{\infty} \sum_{\frac{M}{N} \leq t} \frac{N t+M}{N^{2} y t}\left(\frac{M}{N^{2} y t}\right)^{1+\varepsilon} \ll\|\alpha\|^{2} \frac{M^{1+\varepsilon}}{N^{2}},
$$

where the implied constants depend on the function $h$. Also via (4.19) we see that the remaining sum is dominated by

$$
\mathrm{S}_{2} \ll\|\alpha\|^{2} \sum_{y=1}^{\infty} \sum_{t<\frac{M}{N}} \frac{N t+M}{N^{2} y t}\left(\frac{M}{N^{2} y t}\right)^{\varepsilon} \ll\|\alpha\|^{2} \frac{M^{1+\varepsilon}}{N^{2}},
$$

again with implied constants depending on the Schwartz function $h$. This finishes the considerations of the part of the sum in 4.16 for which $t$ has no upper bound. It remains to deal with the part of the sum for which the summation index $t$ cannot become too large, in which case the argument of the Bessel function is indeed bigger than one. As a consequence employing the approach we used above, relying on the power series expansion of the $J$-Bessel function to separate variables in its argument, is no longer an option, since this would lead to estimates of unsuitable size for our purposes. Therefore we are going to write the $J$ Bessel function as its inverse Mellin transform to separate variables, which will allow us to overcome this issue. Indeed from [8, 6.8 (1), p.326] we know that via the inverse Mellin transform we may rewrite the $J$-Bessel function as

$$
J_{\nu}(x)=\frac{1}{2 \pi \mathrm{i}} \int_{(\sigma)} 2^{s-1} \frac{\Gamma\left(\frac{s+\nu}{2}\right)}{\Gamma\left(\frac{\nu-s+2}{2}\right)} x^{-s} d s, \quad \text { for } \quad-\Re \nu<\Re s=\sigma<3 / 2 .
$$

In the following we adapt an approach to control the size of the inverse Mellin transforms of the $Y$ - and $K$-Bessel functions, presented in the proof of [11, Proposition 9, (see for the proof p. 650-652 therein)] to the present situation. We assume we have $\Re \nu=3 / 4$ and observe that for $-3 / 4<\sigma<3 / 2$ the integrand in (4.22) is holomorphic. Hence instead of integrating along the vertical line at $\sigma$ we may integrate along the contour $\gamma$ connecting $-\varepsilon-\mathrm{i} \infty,-\varepsilon-\mathrm{i}(2+2|\Im \nu|), \varepsilon+1-\mathrm{i}(2+2|\Im \nu|), \varepsilon+1+\mathrm{i}(2+2|\Im \nu|)$, $-\varepsilon+\mathrm{i}(2+2|\Im \nu|)$ and $-\varepsilon+\mathrm{i} \infty$, for a small $\varepsilon>0$. This contour is essentially the same as the one used in the proof in [11]. By Stirling approximation we see that

$$
\frac{\Gamma\left(\frac{s+\nu}{2}\right)}{\Gamma\left(\frac{\nu-s+2}{2}\right)} \ll \frac{e^{\frac{\pi}{2}\left|\Im\left(\frac{\nu-s}{2}\right)\right|}\left(1+\left|\Im\left(\frac{\nu-s}{2}\right)\right|\right)^{\Re\left(\frac{s-\nu}{2}\right)-\frac{1}{2}}}{e^{\frac{\pi}{2}\left|\Im\left(\frac{\nu+s}{2}\right)\right|}\left(1+\left|\Im\left(\frac{\nu+s}{2}\right)\right|\right)^{\frac{1}{2}-\Re\left(\frac{s+\nu}{2}\right)}} .
$$

For the exponential part of this bound it is not hard to show that it can be estimated by $\exp (\pi|\Im \nu| / 2)$. As for the polynomial component of the latter bound we remark that it is exactly the same as the polynomial part of the Stirling estimate of the gamma factors present in the Mellin transform of the $Y$ - and $K$-Bessel function ${ }^{(I)}$. As a consequence with essentially the same argument as executed in the proof of [11, Proposition 9, (see for the proof p. 650-652 therein)], for $x \geq 1$ on every segment of $\gamma$ the integral in (4.22), where of course we integrate along $\gamma$ now instead of the line stated therein, is bounded by $e^{\pi|\Im \nu| / 2}\left(1+|\Im \nu|^{1+\varepsilon}\right) x^{\varepsilon}$, and hence so is $J_{\nu}(x)$. Now we are in a position to determine the size of the sum in 4.16 with small $t$. First we shift the contour in $\mathscr{J}$ to $\Im(w)=-3 / 8$. Now writing the Bessel function as

$$
J_{2 w \mathrm{i}+3 / 4}\left(\frac{4 \pi \sqrt{m n}}{N^{2} y t}\right)=\frac{1}{2 \pi \mathrm{i}} \int_{\gamma} 2^{s-1} \frac{\Gamma\left(\frac{s+2 w \mathrm{i}+3 / 4}{2}\right)}{\Gamma\left(\frac{2 w \mathrm{i}-s+11 / 4}{2}\right)}\left(\frac{4 \pi \sqrt{m n}}{N^{2} y t}\right)^{-s} d s
$$

\footnotetext{
${ }^{(\mathrm{I})}$ For the latter compare for instance with 7.32 .
} 
in $\mathscr{J}$, where $\gamma$ is the contour introduced above, we may estimate the sum in 4.16 we want to treat by

$$
\int_{\mathbb{R}}\left|\frac{h(w-3 \mathrm{i} / 8)(w-3 \mathrm{i} / 8)}{\cosh (\pi(w-3 \mathrm{i} / 8))}\right| \sum_{y, t} \int_{\gamma}\left|\frac{\Gamma\left(\frac{s+2 w \mathrm{i}+3 / 4}{2}\right)}{\Gamma\left(\frac{2 w \mathrm{i}-s+11 / 4}{2}\right)}\right| \frac{\left|\mathcal{F}_{y, t}(\alpha ; s)\right|}{\left(N^{2} y t\right)^{1-\Re s}}|d s| d w
$$

where the $y, t$-summations are as described for the case we deal with at the moment; we set the stage for an application of a large sieve for additive characters as before, by applying the Cauchy Schwarz inequality to $\mathcal{F}_{y, t}(\alpha ; s)$ and afterwards again enlarge the resulting sums in each factor to all residue classes: Then with the large sieve estimate for additive characters from [14, Theorem 7.7], for the same family of harmonics as the ones which we used earlier in this proof, we see

$$
\mathcal{F}_{y, t}(\alpha ; s) \ll\|\alpha\|^{2}(N t+M) M^{-\Re s} .
$$

Hence by the remarks made previously about bounding the contour integral representing the $J$-Bessel function we therefore find that 4.23 is dominated by

$$
\int_{\mathbb{R}}|h(w-3 \mathrm{i} / 8)|\left(1+|w|^{2+\varepsilon}\right) d w \cdot \sum_{y, t} \frac{N t+M}{N^{2} y t}\left(\frac{M}{N^{2} y t}\right)^{\varepsilon}\|\alpha\|^{2},
$$

for a small $\varepsilon>0$. Again the integral converges, since the function $h$ is a Schwartz function that decays very fast, as described in Section 2.4, and thus we further estimate the latter bound by

$$
\|\alpha\|^{2} \sum_{y=1}^{\infty} \sum_{t<\frac{4 \pi M}{N^{2} y}} \frac{N t+M}{N^{2} y t}\left(\frac{M}{N^{2} y t}\right)^{\varepsilon} \ll\|\alpha\|^{2} \frac{M^{1+\varepsilon}}{N^{2}} .
$$

Finally we combine the last estimate with the ones we found in 4.20 and 4.21 to conclude via 4.17 that the sum in 4.16, and therefore the expression in 4.15, is dominated by

$$
\|\alpha\|^{2} \frac{M^{1+\varepsilon}}{N^{2}} \text {. }
$$

Thus as explained in the beginning of the proof this is enough to control $\Phi^{\text {thin }}(\alpha)$ with the very same upper bound and hence this finishes the argument.

\subsection{The size of the error term}

This section is dedicated to proving Lemma 4.4. This means we want to show the bound

$$
\Phi_{t>T}(\alpha) \ll\|\alpha\|^{2}\left(1+\frac{M}{N^{2}}\right) .
$$

under the assumption that $T \geq M / N>1$ and for an arbitrary $\alpha \in \mathbb{C}_{M}^{\infty}$. For the following argument supporting this statement we are going to follow the same strategy and outline as used in the proof of [15, Lemma 6.1, (proof and statement on p.553)].

Proof. We begin be estimating the term $\Phi_{t>T}(\alpha)$ directly. Inserting into its definition in 4.9 ) the expression for $\phi(m, n, t)$ from $(3.6)$, we find that

$$
\Phi_{t>T}(\alpha) \ll \sum_{y=1}^{\infty} \frac{\Phi_{t>T}(\alpha ; y)}{N y}
$$


where the function $\Phi_{t>T}(\alpha ; y)$ is given by

$$
\sum_{t>T} \frac{1}{t} \sum_{\substack{x \bmod t \\(x(x+y N), t)=1}}\left|\sum_{M<m, n \leq 2 M} \bar{a}_{m} a_{n} e\left(\frac{m \bar{x}-n \overline{(x+y N)}}{t}+\frac{m+n}{y N t}\right) \mathscr{J}\left(\frac{4 \pi \sqrt{m n}}{y N t}\right)\right|,
$$

where by positivity we have enlarged the $t$-summation to run over all $t>T$, regardless of the index being devisible by $N$ or not. Furthermore the bound in (4.24) relies on the fact that we know $\mathrm{P}(g)=2 \Re(g) \ll|g|$. Now for technical reasons we need the $J$-Bessel function appearing in this formula to have an index with a real part bigger than one. Hence we shift the contour in $\mathscr{J}$ to $\Im(w)=-(\varepsilon+1) / 2$. We can do this without picking up the residue of the pole of the spectral measure $d_{\mathrm{spec}} w$ at $z=-\mathrm{i} / 2$ since we demand that the function $h$ in the integral transform $\mathscr{J}$ has a zero at at this point to precisely counteract that pole (see Section 2.4 for the requirements of the function $h$ ). In the present scenario we encounter that $t>T \geq M / N$, which implies that

$$
\frac{m+n}{y N t} \ll 1 \text { and } \frac{4 \pi \sqrt{m n}}{y N t} \ll 1 .
$$

These two bounds say that the arguments of the $J$-Bessel function and of the exponential, that does not involve residue classes in its argument, are uniformly bounded by a constant and therefore we may rely in both cases on their expansion as a power series to separate variables, without increasing the upcoming estimates by more than a constant, which is desirable in our situation. For the Bessel function we know from [10, 8.402] that

$$
J_{\nu}(x)=\sum_{k=0}^{\infty} \frac{(-1)^{k}}{k ! \Gamma(\nu+k+1)}\left(\frac{x}{2}\right)^{2 k+\nu}
$$

for $x \in \mathbb{R}_{>0}$. Employing this series and the well known series representing the exponential function we separate variables in $\Phi_{t>T}(\alpha ; y)$ and, after using the functional equation of the gamma function, obtain for it the bound

$$
\sum_{j, l, k=0}^{\infty} \frac{(2 \pi)^{2 k+1+\varepsilon}}{j ! l ! k !(k+1) !(N y)^{j+l+2 k+1+\varepsilon}} \int_{\mathbb{R}}\left|\frac{\mathcal{E}_{j, l, k}(\alpha ; w) h(w-\mathrm{i}(1+\varepsilon) / 2)(1+|w|)}{\cosh (\pi(w-\mathrm{i}(1+\varepsilon) / 2)) \Gamma(2 \mathrm{i} w+1+\varepsilon)}\right| d w ;
$$

by the Cauchy Schwarz inequality and then enlarging both resulting summations over the residue classes by positivity to all invertible ones, we see the bound

$$
\begin{aligned}
\mathcal{E}_{j, l, k}(\alpha ; w) \ll\left(\sum_{t>T} \frac{1}{t^{j+l+2 k+2+\varepsilon}} \sum_{x \bmod t}^{*}\left|\sum_{M<m \leq 2 M} \tilde{A}_{m}^{k, j}(w) e\left(\frac{m x}{t}\right)\right|^{2}\right)^{1 / 2} \cdot \\
\cdot\left(\sum_{t>T} \frac{1}{t^{j+l+2 k+2+\varepsilon}} \sum_{x \bmod t}^{*}\left|\sum_{M<n \leq 2 M} A_{n}^{k, l}(w) e\left(\frac{n x}{t}\right)\right|^{2}\right)^{1 / 2},
\end{aligned}
$$

where we define

$$
\tilde{A}_{m}^{k, j}(w):=\bar{a}_{m} m^{k+j+(1+\varepsilon) / 2+\mathrm{i} w} \quad \text { and } \quad A_{n}^{k, l}(w):=a_{n} n^{k+l+(1+\varepsilon) / 2+\mathrm{i} w} .
$$

The next task is to estimate both factors in the bound 4.26 further. Both may be treated by the same means and we will therefore only demonstrate one case. The idea is to split the range of the $t$-summation 
into intervals of increasing length and apply a large sieve estimate for additive characters to each interval. Concretely we rewrite for instance the square of the first factor in 4.26 as

$$
\sum_{u=0}^{\infty} \sum_{2^{u}} \frac{1}{t^{j+l \leq 2^{u+1} T}} \sum_{x \bmod t}^{*}\left|\sum_{M<m \leq 2 M} A_{m}^{k, j}(w) e\left(\frac{m x}{t}\right)\right|^{2}
$$

and apply the large sieve inequality from [14, Theorem 7.11] to find for it the upper bound

$$
M^{2(k+j)+1+\varepsilon}\|\alpha\|^{2} \sum_{u=0}^{\infty} \frac{2^{2 u} T^{2}+M}{\left(2^{u} T\right)^{j+l+2 k+2+\varepsilon}} \ll \frac{M^{2(k+j)+1+\varepsilon}}{T^{j+l+2 k+2+\varepsilon}}\left(T^{2}+M\right)\|\alpha\|^{2} .
$$

One establishes a similar bound for the second factor in 4.26 and thus we conclude for the whole expression

$$
\mathcal{E}_{j, l, k}(\alpha ; w) \ll\|\alpha\|^{2}\left(T^{2}+M\right) \frac{M^{2 k+j+l+1+\varepsilon}}{T^{j+l+2 k+2+\varepsilon}}
$$

We employ the latter bound in 4.25). Since the arguments of the described exponentials and the Bessel function are bounded uniformly by a constant, so are their series expansions. Hence the sums in $(4.25)$ are absorbed into the implied constant. By Stirling approximation we have

$$
\Gamma(2 \mathrm{i} w+1+\varepsilon)^{-1} \ll e^{\pi|w|}(1+|w|)^{-(1 / 2+\varepsilon)}
$$

and thus recalling that $h$ is a Schwartz function satisfying the properties specified in Section 2.4, via (4.25) we immediately conclude the upper bound

$$
\Phi_{t>T}(\alpha ; y) \ll\left(N+\frac{M}{N}\right)\left(\frac{M}{N T}\right)^{\varepsilon} \frac{\|\alpha\|^{2}}{y^{1+\varepsilon}},
$$

using that we assume $T \geq M / N$. The implied constant depends of course on the function $h$. Inserting the latter estimate in 4.24 we immediately arrive at the desired bound for $\Phi_{t>T}(\alpha)$, which finishes the proof.

\subsection{Estimating the second main term $\mathscr{M}^{0}(\alpha)$}

In this section we want to estimate the second main term $\mathscr{M}^{0}(\alpha)$, which we defined earlier in this chapter in 4.12. This term originates from the asymptotic established in Theorem 3.2 for $\phi(m, n, t)$ and is the result of our efforts to remove the cut-off function $\varrho$, which was necessary to introduce in the first place for technical reasons (see Remark 3.4). Via 4.11) and 4.10 the term $\mathscr{M}^{0}(\alpha)$ is then in principle featured in the asymptotic formula for $\Phi(\alpha)$. However Lemma 4.5 tells us that in fact this term is not too large and thus is consumed by the error term we have already established in $(4.10)$. Precisely the lemma says that for $H=327 T^{1+\delta}$, where we set $T=M / N>1$, we can show that

$$
\mathscr{M}^{0}(\alpha) \ll\|\alpha\|^{2}\left(1+\frac{M}{N^{2}}\right) M^{\varepsilon},
$$

for any $\alpha \in \mathbb{C}_{M}^{\infty}$. The implied constant in this estimate depends on the Schwartz function $h$. We will now give a detailed proof in support of Lemma 4.5. This argument which we are going to present is based on the strategy and outline of the proof showing [15, Corollary 9.1, (for statement and proof see p. 555-556)]. 
We proceed towards our aim of establishing the above estimate for the second main term by writing it as the sum

$$
\mathscr{M}^{0}(\alpha)=\mathscr{M}_{1}^{0}(\alpha)+\mathscr{M}_{2}^{0}(\alpha)
$$

where we define the two terms by

$$
\mathscr{M}_{1}^{0}(\alpha):=\frac{1}{N} \sum_{t \leq T} \frac{1}{t^{2}} \sum_{M<m, n \leq 2 M} \bar{a}_{m} a_{n} S(0, m-n, t) \int_{0}^{2 H} f\left(\frac{y}{H}\right) \mathscr{I}\left(\frac{m}{N t}, \frac{n}{N t}, \frac{-y}{t}\right) d y
$$

and

$$
\mathscr{M}_{2}^{0}(\alpha):=\frac{1}{N} \sum_{t \leq T} \frac{1}{t^{2}} \sum_{M<m, n \leq 2 M} \bar{a}_{m} a_{n} S(0, m-n, t) \int_{0}^{2 H} f\left(\frac{y}{H}\right) \mathscr{I}\left(\frac{m}{N t}, \frac{n}{N t}, \frac{y}{t}\right) d y .
$$

We recall that we assume that $f$ is an even function. For convenience we recall the definition of the function $\mathscr{I}$, as for instance stated in Theorem 4.2. We have for negative last argument

$$
\mathscr{I}\left(\frac{m}{N t}, \frac{n}{N t}, \frac{-y}{t}\right)=\frac{8 \mathrm{i}}{\pi} \int_{\mathbb{R}} x h(x) Y_{2 \mathrm{i} x}\left(\frac{4 \pi}{t} \sqrt{\frac{y \max \{m, n\}}{N}}\right) K_{2 \mathrm{i} x}\left(\frac{4 \pi}{t} \sqrt{\frac{y \min \{m, n\}}{N}}\right) d x
$$

and if the last argument is positive

$$
\mathscr{I}\left(\frac{m}{N t}, \frac{n}{N t}, \frac{y}{t}\right)=-4 \mathrm{i} \int_{\mathbb{R}} \frac{x h(x)}{\sinh (\pi x) \cosh (\pi x)} J_{2 \mathrm{i} x}\left(\frac{4 \pi}{t} \sqrt{\frac{y m}{N}}\right) \Im\left(J_{2 \mathrm{i} x}\left(\frac{4 \pi}{t} \sqrt{\frac{y n}{N}}\right)\right) d x .
$$

We remark that we also know that the function $\mathscr{I}(*, *, 0)$ takes a form different from the ones just stated for negative/positive last argument. The precise evaluation is also given in Theorem 4.2 In the following considerations of this chapter we may however disregard this, since we integrate over the last argument of the function $\mathscr{I}$ in the definitions of $\mathscr{M}_{1}^{0}(\alpha)$ and $\mathscr{M}_{2}^{0}(\alpha)$ and one discrete value is a measure zero set, that may be excluded from the range of integration without changing the value of the integral itself in the Lebesgue sense. Now, in order to determine the size of $\mathscr{M}^{0}(\alpha)$, we will treat both of its parts, $\mathscr{M}_{1}^{0}(\alpha)$ and $\mathscr{M}_{2}^{0}(\alpha)$, separately and establish the upper bounds

$$
\mathscr{M}_{1}^{0}(\alpha), \mathscr{M}_{2}^{0}(\alpha) \ll\|\alpha\|^{2} M^{\varepsilon}\left(1+\frac{M}{N^{2}}\right),
$$

showing that $\mathscr{M}^{0}(\alpha)$ via 4.27 indeed admits the estimate, which we claimed in Lemma 4.5 A key step towards establishing the latter estimates is a sieve inequality for some Bessel functions in the style of [15. Lemma 9.1]. For the $Y$ and the $J$-Bessel function of certain complex index $\nu$ we obtain the following.

Lemma 4.6. Consider $1 \leq Z \leq T / 2$ and $\Delta \leq R \leq H$, for $\Delta=\left(32 \pi^{2} T\right)^{-1}$, where $T=M / N>1$ and $H=327 T^{1+\delta}$, just as in the conditions of Lemma 4.5. For any $\alpha \in \mathbb{C}_{M}^{\infty}$ define

$$
\mathcal{M}_{B}(Z, R ; \nu):=\sum_{Z<t \leq 2 Z} \int_{R}^{2 R} \sum_{x \bmod t}^{*}\left|\sum_{M<n \leq 2 M} a_{n} e\left(\frac{x n}{t}\right) B_{\nu}\left(\frac{4 \pi}{t} \sqrt{\frac{y n}{N}}\right)\right|^{2} d y .
$$

Then we have

$$
\mathcal{M}_{B}(Z, R ; \nu) \ll\|\alpha\|^{2} N M^{\varepsilon} Z^{2}\left(1+\left(\frac{Z^{2} R}{N M}\right)^{1 / 2}\right)\left(1+|\Im \nu|^{9}\right) e^{2 \pi|\Im \nu|} .
$$

The function $B_{\nu}(x)$ denotes either the $Y$ - or the J-Bessel function with index $\nu \in \mathbb{C}$ with $|\Re \nu|<1 / 2$ and of argument $x \in \mathbb{R}_{>0}$. 
We will justify this result in Section 4.7. For now we will employ it to make progress with bounding $\mathscr{M}^{0}(\alpha)$. As announced this is going to be a result of the separate estimates for $\mathscr{M}_{1}^{0}(\alpha)$ and $\mathscr{M}_{2}^{0}(\alpha)$, as claimed in 4.28, which we present in detail now.

\subsubsection{The size of $\mathscr{M}_{1}^{0}(\alpha)$}

In the following we will bound $\mathscr{M}_{1}^{0}(\alpha)$. For technical reasons we split the $y$ integral in its definition at $\Delta=\left(32 \pi^{2} T\right)^{-1}$ and estimate the resulting two sums $\mathscr{M}_{1, y<\Delta}^{0}(\alpha)$ and $\mathscr{M}_{1, y \geq \Delta}^{0}(\alpha)$, i.e. respectively

$$
\frac{1}{N} \sum_{t \leq T} \frac{1}{t^{2}} \int_{0}^{\Delta} \ldots d y \text { and } \frac{1}{N} \sum_{t \leq T} \frac{1}{t^{2}} \int_{\Delta}^{2 H} \ldots d y
$$

that sum together to $\mathscr{M}_{1}^{0}(\alpha)$, individually. We will treat the case of small $y$ first. To get rid of the max $/ \min$ functions in the arguments of the Bessel functions, we define a smooth function $\kappa_{1}: \mathbb{R} \rightarrow \mathbb{R}_{\geq 0}$ supported on $(-1, \infty)$ with $\kappa_{1}(0)=1 / 2$ and $\kappa_{1}(x)=1$ for $x \geq 1$. Inserting this function into $\mathscr{M}_{1, y<\Delta}^{0}(\alpha)$ shows that by symmetry it suffices to bound

$$
\begin{array}{r}
\frac{1}{N} \sum_{t \leq T} \frac{1}{t^{2}} \int_{0}^{\Delta} f\left(\frac{y}{H}\right) \int_{\mathbb{R}} w h(w) \sum_{M<m, n \leq 2 M} \kappa_{1}(m-n) \bar{a}_{m} a_{n} S(0, m-n, t) . \\
\cdot Y_{2 \mathrm{i} w}\left(\frac{4 \pi}{t} \sqrt{\frac{y m}{N}}\right) K_{2 \mathrm{i} w}\left(\frac{4 \pi}{t} \sqrt{\frac{y n}{N}}\right) d w d y
\end{array}
$$

In the latter we estimate the Kloosterman sum and $\kappa_{1}$ trivially. Furthermore we recall that the cut-off function satisfies $f(x)=1$, for $-1 \leq x \leq 1$. Hence $\mathscr{M}_{1, y<\Delta}^{0}(\alpha)$ is dominated by

$$
\frac{1}{N} \sum_{t \leq T} \frac{1}{t} \int_{0}^{\Delta} \int_{\mathbb{R}}|w h(w)| \sum_{M<m, n \leq 2 M}\left|\bar{a}_{m} a_{n} Y_{2 \mathrm{i} w}\left(\frac{4 \pi}{t} \sqrt{\frac{y m}{N}}\right) K_{2 \mathrm{i} w}\left(\frac{4 \pi}{t} \sqrt{\frac{y n}{N}}\right)\right| d w d y
$$

We observe that for $y \leq \Delta$ the arguments of the Bessel functions both do not exceed one. Thus from [11, Proposition 9] we find

$$
Y_{2 \mathrm{i} w}\left(\frac{4 \pi}{t} \sqrt{\frac{y m}{N}}\right) K_{2 \mathrm{i} w}\left(\frac{4 \pi}{t} \sqrt{\frac{y n}{N}}\right) \ll\left(\left(1+|w|^{2}\right) \frac{t^{2} N}{y \sqrt{m n}}\right)^{\delta},
$$

for any small $\delta>0$. Applying this bound in 4.29) quickly gives

$$
\mathscr{M}_{1, y<\Delta}^{0}(\alpha) \ll M^{\varepsilon}\|\alpha\|^{2} .
$$

The implied constant depends on the Schwartz function $h$. Now we need to focus on estimating the part of $\mathscr{M}_{1}^{0}(\alpha)$, where $y$ in the integration is at least $\Delta$ away from zero. More precisely we aim to bound $\mathscr{M}_{1, y \geq \Delta}^{0}(\alpha)$, which is

$$
\begin{aligned}
& \frac{8 \mathrm{i}}{N \pi} \sum_{t \leq T} \frac{1}{t^{2}} \int_{\Delta}^{2 H} f\left(\frac{y}{H}\right) \int_{\mathbb{R}} w h(w) \sum_{M<m, n \leq 2 M} \bar{a}_{m} a_{n} S(0, m-n, t) . \\
& \cdot Y_{2 \mathrm{i} w}\left(\frac{4 \pi}{t} \sqrt{\frac{y \max \{m, n\}}{N}}\right) K_{2 \mathrm{i} w}\left(\frac{4 \pi}{t} \sqrt{\frac{y \min \{m, n\}}{N}}\right) d w d y .
\end{aligned}
$$


In order to be able to work with the above expression more easily, as a first step we want to get rid of the $\max / \min$ functions in the arguments of the Bessel functions. We facilitate this task by introducing a smooth function

$$
\kappa: \mathbb{R} \rightarrow \mathbb{R}_{\geq 0} \text { with } \operatorname{supp}(\kappa) \subseteq[-1 / M, 3 / 2],
$$

which satisfies $\kappa(0)=1 / 2$ and equals one on $[1 / M, 1]$. Furthermore the function and its derivatives admit the bounds $\kappa^{(j)}(x) \ll M^{j}$, on $[-1 / M, 1 / M]$, and $\kappa^{(j)}(x) \ll 1$, on $[1,3 / 2]$, both for any $j \geq 0$. Ultimately we want to be able to separate variables in the argument of the function $\kappa$ by means of the Fourier transform without adding too much to the general size of the bound of the whole term, which we try to estimate. Thus we need the inverse Fourier transform to converge absolutely with a bound that is small enough in this context. To see this we have to investigate the size of the Fourier transform $\hat{\kappa}$ of $\kappa$. Performing integration by parts and also estimating trivially we find the upper bound

$$
\hat{\kappa}(y) \ll \min \left\{1, \frac{1}{|y|}, \frac{M}{|y|^{2}}\right\} \ll \min \left\{1, \frac{M^{\varepsilon}}{|y|^{1+\varepsilon}}\right\},
$$

where we used that $\min \{A, B\} \leq A^{x} B^{1-x}$, for any $A, B \geq 0$ and $0 \leq x \leq 1$. This guarantees that the inverse Fourier transform converges absolutely with the bound

$$
\int_{\mathbb{R}}|\hat{\kappa}(y)| d y \ll M^{\varepsilon},
$$

which means that the price of separating variables in $\kappa$ is only an epsilon power of $M$, i.e. for our purposes acceptable. Equipped with this function we may now rewrite the summations over $m$ and $n$ in $\mathscr{M}_{1, y \geq \Delta}^{0}(\alpha)$, as stated in 4.31, in the form

$$
\begin{aligned}
\sum_{M<m, n \leq 2 M} \bar{a}_{m} a_{n} S(0, m-n, t) \kappa\left(\frac{m-n}{M}\right) Y_{2 \mathrm{i} w}\left(\frac{4 \pi}{t} \sqrt{\frac{y m}{N}}\right) K_{2 \mathrm{i} w}\left(\frac{4 \pi}{t} \sqrt{\frac{y n}{N}}\right) \\
+\sum_{M<m, n \leq 2 M} \bar{a}_{m} a_{n} S(0, m-n, t) \kappa\left(\frac{n-m}{M}\right) Y_{2 \mathrm{i} w}\left(\frac{4 \pi}{t} \sqrt{\frac{y n}{N}}\right) K_{2 \mathrm{i} w}\left(\frac{4 \pi}{t} \sqrt{\frac{y m}{N}}\right) .
\end{aligned}
$$

As already announced we now separate variables in the argument of $\kappa$ by means of the Fourier transform. Furthermore we express the $K$-Bessel function via its inverse Mellin transform (cp. Subsection 7.2.1 and in particular (7.31) ) in the form

$$
K_{2 \mathrm{i} w}(x)=\frac{1}{2 \pi \mathrm{i}} \int_{(\sigma)} 2^{s-2} \Gamma\left(\frac{s-2 \mathrm{i} w}{2}\right) \Gamma\left(\frac{s+2 \mathrm{i} w}{2}\right) x^{-s} d s,
$$

which converges absolutely for any $\sigma>0$ (cp. Lemma 7.9). We will justify the appropriate choice of the parameter $\sigma$ at a later stage. For now we proceed with our estimation and observe that by symmetry of the two terms added together in (4.34) the expression (4.31) is thus dominated by

$$
\begin{aligned}
& \frac{1}{N} \int_{\mathbb{R}}|w h(w)| \int_{\mathbb{R}}|\hat{\kappa}(l)| \int_{(\sigma)}\left|\Gamma\left(\frac{s-2 \mathrm{i} w}{2}\right) \Gamma\left(\frac{s+2 \mathrm{i} w}{2}\right)\right| \int_{\Delta}^{2 H} \sum_{t \leq T} \frac{1}{t^{2}} \sum_{x \bmod t}^{*} \cdot \\
& \left|\sum_{M<m \leq 2 M} b_{m}(l) e\left(\frac{x m}{t}\right) Y_{2 \mathrm{i} w}\left(\frac{4 \pi}{t} \sqrt{\frac{y m}{N}}\right)\right|\left|\sum_{M<n \leq 2 M} \bar{b}_{n}(l) e\left(\frac{-x n}{t}\right)\left(\frac{2 \pi}{t} \sqrt{\frac{y n}{N}}\right)^{-s}\right| d y|d s| d l d w,
\end{aligned}
$$


both relying on the abbreviation $b_{h}(l):=\bar{a}_{h} e(l h / M)$ and where $\hat{\kappa}$ denotes again the Fourier transform of $\kappa$. To obtain the latter estimate we opened the Kloosterman sum and point out that by definition the cut-off function satisfies

$$
f(x) \ll 1 \text { for } x \in \mathbb{R} .
$$

At this stage we will begin to bring the large sieve type inequality as provided by Lemma 4.6 into the picture. For this result to become applicable we have to consider a scenario where the $y$ variable in the integration as well as the summation in $t$ run in intervals. Hence we estimate the last bound for $\mathscr{M}_{1, y \geq \Delta}^{0}(\alpha)$ by summing intervals of decreasing length up to the full range of the $y$-integration and the $t$-summation. Then applying the Cauchy Schwarz inequality we see that 4.35 does not exceed

$$
\frac{1}{N T^{2}} \int_{\mathbb{R}} \int_{\mathbb{R}} \int_{(\sigma)} \ldots \sum_{j=0}^{\left\lceil\frac{\log T}{\log 2}-1\right\rceil} 2^{2 j} \sum_{u=0}^{\left\lceil\frac{\log (H / \Delta)}{\log 2}\right.} \int_{\frac{H}{2^{u}}}^{\frac{2 H}{2^{u}}} \mathcal{Y}_{\mathrm{int}}^{l, y, j}(\alpha ; w)^{1 / 2} \mathcal{K}_{\mathrm{int}}^{l, y, j}(\alpha ; s)^{1 / 2} d y|d s| d l d w
$$

where we define

$$
\mathcal{Y}_{\mathrm{int}}^{l, y, j}(\alpha ; w):=\sum_{\frac{T}{2^{j}+1}<t \leq \frac{T}{2^{j}}} \sum_{x \bmod t}^{*}\left|\sum_{M<n \leq 2 M} b_{n}(l) e\left(\frac{x n}{t}\right) Y_{2 \mathrm{i} w}\left(\frac{4 \pi}{t} \sqrt{\frac{y n}{N}}\right)\right|^{2}
$$

and

$$
\mathcal{K}_{\mathrm{int}}^{l, y, j}(\alpha ; s):=\sum_{\frac{T}{2^{j+1}}<t \leq \frac{T}{2^{j}}} \sum_{x \bmod t}^{*}\left|\sum_{M<n \leq 2 M} \bar{b}_{n}(l) e\left(\frac{-x n}{t}\right)\left(\frac{2 \pi}{t} \sqrt{\frac{y n}{N}}\right)^{-s}\right|^{2}
$$

We will proceed with bounding 4.36 further by turning our attention first towards the term $\mathcal{K}_{\text {int }}^{l, y, j}(\alpha ; s)$. Based on the large sieve inequality for additive characters as given in [14, Theorem 7.11] we establish quickly that the integral

$$
\int_{(\sigma)}\left|\Gamma\left(\frac{s-2 \mathrm{i} w}{2}\right) \Gamma\left(\frac{s+2 \mathrm{i} w}{2}\right)\right| \mathcal{K}_{\mathrm{int}}^{l, y, j}(\alpha ; s)^{1 / 2}|d s|
$$

is dominated by

$$
\|\alpha\|\left(\frac{T^{2} N}{2^{2 j} M y}\right)^{\sigma / 2}\left(\frac{T}{2^{j}}+M^{1 / 2}\right) \frac{1+|w|^{\max \{3(\sigma-1) / 2, \sigma\}}}{e^{\pi|w| / 2}},
$$

where Lemma 7.9 provides the upper bound for the contour integral, uniform in the imaginary part of the index of the $K$-Bessel function. Inserting the estimate from 4.37) into (4.36) and once more employing the Cauchy Schwarz inequality we find that $\mathscr{M}_{1, y \geq \Delta}^{0}(\alpha)$ is bounded by

$$
\begin{aligned}
\frac{\|\alpha\| H^{1 / 2}}{N T^{2}} & \left(\frac{T^{2} N}{M H}\right)^{\sigma / 2} \int_{\mathbb{R}} \frac{|h(w)|\left(|w|+|w|^{\max \{(3 \sigma-1) / 2, \sigma+1\}}\right)}{e^{\pi|w| / 2}} \int_{\mathbb{R}}|\hat{\kappa}(l)| \\
& \sum_{j=0}^{\left\lceil\frac{\log T}{\log 2}-1\right\rceil} \sum_{u=0}^{\left.\frac{\log (H / \Delta)}{\log 2}\right\rceil} 2^{j(2-\sigma)+u(\sigma-1) / 2}\left(\frac{T}{2^{j}}+M^{1 / 2}\right) \mathcal{M}_{Y}\left(\frac{T}{2^{j+1}}, \frac{H}{2^{u}} ; 2 \mathrm{i} w\right)^{1 / 2} d l d w,
\end{aligned}
$$


where the function $\mathcal{M}_{Y}$ is as defined in Lemma 4.6. Although not specifically highlighted here we note that it depends on the integration variable $l$. Now we utilise the large sieve type bound as provided by Lemma 4.6 to see that 4.38 ) is at most

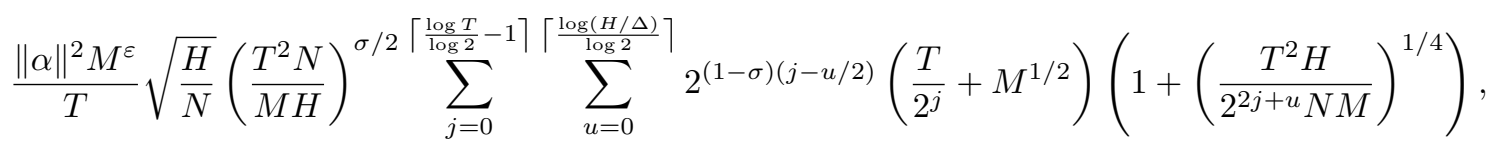

where we exploit that $h$ is a Schwartz function satisfying the decay properties as explained in Section 2.4 . which ensure absolute convergence of the $w$ integral, and the estimate given in 4.33 to control the integral over the absolute value of the Fourier transform $\hat{\kappa}$. Now, with the choice $\sigma=1-\varepsilon$, for any small $\varepsilon>0$, we derive for the previous bound the estimate

$$
\|\alpha\|^{2} M^{\tilde{\varepsilon}} \sum_{j=0}^{\left\lceil\frac{\log T}{\log 2}-1\right\rceil} \sum_{u=0}^{\infty} 2^{\varepsilon(j-u / 2)}\left(\frac{M^{1 / 2}}{2^{j} N}+1\right)\left(1+\left(\frac{M}{2^{j+u / 2} N^{2}}\right)^{1 / 2}\right),
$$

where we recall that $T=M / N$ and $H=327 T^{1+\delta}$. From the latter upper bound we then finally conclude that

$$
\mathscr{M}_{1, y \geq \Delta}^{0}(\alpha) \ll\|\alpha\|^{2} M^{\varepsilon}\left(1+\frac{M}{N^{2}}+\frac{M^{1 / 2}}{N}\right) .
$$

Combining this result with the estimate obtained in 4.30 , we see that the whole of $\mathscr{M}_{1}^{0}(\alpha)$ admits the bound as claimed in 4.28 . This finishes the treatment of the first part of the term $\mathscr{M}^{0}(\alpha)$.

\subsubsection{The size of $\mathscr{M}_{2}^{0}(\alpha)$}

In order to establish the desired bound for $\mathscr{M}^{0}(\alpha)$ it remains to show that $\mathscr{M}_{2}^{0}(\alpha)$ satisfies the upper bound in 4.28 , too. As in the previous case we split the $y$-integration in $\mathscr{M}_{2}^{0}(\alpha)$ again at $\Delta=\left(32 \pi^{2} T\right)^{-1}$ and consider the two resulting integrals

$$
\frac{1}{N} \sum_{t \leq T} \frac{1}{t^{2}} \int_{0}^{\Delta} \ldots d y \text { and } \frac{1}{N} \sum_{t \leq T} \frac{1}{t^{2}} \int_{\Delta}^{2 H} \ldots d y
$$

individually. Similarly to the case of $\mathscr{M}_{1}^{0}(\alpha)$, treated in the previous subsection, we denote those integrals by $\mathscr{M}_{2, y<\Delta}^{0}(\alpha)$ and $\mathscr{M}_{2, y \geq \Delta}^{0}(\alpha)$ respectively. We will deal with the term $\mathscr{M}_{2, y<\Delta}^{0}(\alpha)$ for small $y$, not exceeding $\Delta$, first. To this end we shift the contour in $\mathscr{I}$ to $\Im(w)=-1 / 8$. Hence estimating the Kloosterman sum trivially, we immediately obtain for it the bound

$$
\begin{aligned}
\frac{1}{N} \sum_{t \leq T} \frac{1}{t} \int_{0}^{\Delta} f\left(\frac{y}{H}\right) \int_{\mathbb{R}}\left|\frac{(w-\mathrm{i} / 8) h(w-\mathrm{i} / 8)}{\sinh (2 \pi(w-\mathrm{i} / 8))}\right| \\
\sum_{M<m, n \leq 2 M}\left|\bar{a}_{m} a_{n} J_{2 \mathrm{i} w+1 / 4}\left(\frac{4 \pi}{t} \sqrt{\frac{y m}{N}}\right) \sum_{ \pm} \mp J_{ \pm 2 \mathrm{i} w \pm 1 / 4}\left(\frac{4 \pi}{t} \sqrt{\frac{y n}{N}}\right)\right| d w d y .
\end{aligned}
$$

For clarity we have stated the cut-off function $f$ here again, however in this particular scenario it is in fact obsolete since it satisfies $f(x)=1$, for $-1 \leq x \leq 1$. Furthermore with Lemma 7.1 we estimate

$$
J_{2 \mathrm{i} w+1 / 4}\left(\frac{4 \pi}{t} \sqrt{\frac{y m}{N}}\right) J_{ \pm 2 \mathrm{i} w \pm 1 / 4}\left(\frac{4 \pi}{t} \sqrt{\frac{y n}{N}}\right) \ll e^{2 \pi|w|},
$$


again observing that for $y \leq \Delta$ the arguments of the Bessel functions are both bounded by one. Now it is easy to see that $\sinh (2 \pi(w-\mathrm{i} / 8)) \gg \exp (2 \pi|w|)$, for all $w \in \mathbb{R}$, and recalling from Section 2.4 that the function $h$ is assumed to decay rapidly for large argument, based on 4.39 we conclude

$$
\mathscr{M}_{2, y<\Delta}^{0}(\alpha) \ll M^{\varepsilon}\|\alpha\|^{2},
$$

where the implied constant once more is influenced by the choice of $h$. We turn our attention to $\mathscr{M}_{2, y>\Delta}^{0}(\alpha)$. In a first step we shift the contour in the $w$ integral to $\Im(w)=-1 / 8$. Now inserting the definition of the Kloosterman sum and applying the Cauchy Schwarz inequality appropriately, we find that this term under investigation is bounded by

$$
\frac{1}{N} \int_{\mathbb{R}}\left|\frac{(w-\mathrm{i} / 8) h(w-\mathrm{i} / 8)}{\sinh (2 \pi(w-\mathrm{i} / 8))}\right| \int_{\Delta}^{2 H} \sum_{t \leq T} \frac{1}{t^{2}} \sum_{x \bmod t}^{*} \mid \sum_{M<n \leq 2 M} a_{n} e\left(\frac{x n}{t}\right) J_{2 \mathrm{i} w+1 / 4}\left(\frac{4 \pi}{t} \sqrt{\frac{y n}{N}}\right)^{2} d y d w
$$

where we highlight the fact that the cut-off function admits the bound

$$
f(x) \ll 1, \text { for } x \in \mathbb{R} .
$$

From the latter estimate in 4.41 we would like to deduce the bound for $\mathscr{M}_{2, y \geq \Delta}^{0}(\alpha)$ of desired quality by an application of Lemma 4.6 In order to be able to apply the large sieve type inequality given therein for the $J$-Bessel function, we have to break down the range the $y$-integration and the $t$-summation into smaller intervals of decreasing length and sum over those to the full range in each case. Precisely we obtain that (4.41) does not exceed

$$
\frac{1}{N T^{2}} \int_{\mathbb{R}} \frac{(1+|w|)|h(w-\mathrm{i} / 8)|}{e^{2 \pi|w|}} \sum_{j=0}^{\left\lceil\frac{\log T}{\log 2}-1\right\rceil} 2^{2 j} \sum_{u=0}^{\left\lceil\frac{\log (H / \Delta)}{\log 2}\right\rceil} \mathcal{M}_{J}\left(\frac{T}{2^{j+1}}, \frac{H}{2^{u}} ; 2 \mathrm{i} w+1 / 4\right) d w,
$$

where once more we employ the lower bound $\sinh (2 \pi(w-\mathrm{i} / 8)) \gg \exp (2 \pi|w|)$, for all $w \in \mathbb{R}$. Here the function $\mathcal{M}_{J}$, here in case for the $J$-Bessel function, is as defined in Lemma 4.6, the result from which we further deduce the upper bound

$$
\|\alpha\|^{2} M^{\varepsilon} \sum_{j=0}^{\left\lceil\frac{\log T}{\log 2}-1\right\rceil\lceil} \sum_{u=0}^{\left.\frac{\log (H / \Delta)}{\log 2}\right\rceil}\left(1+\frac{M}{2^{j+u / 2} N^{2}}\right),
$$

upon invoking that $T=M / N$ and $H=327 T^{1+\delta}$ and where the $w$ integral converges due to the properties of the function $h$ specified in Section 2.4. Thus based on the latter upper bound we conclude at once that

$$
\mathscr{M}_{2, y \geq \Delta}^{0}(\alpha) \ll\|\alpha\|^{2} M^{\varepsilon}\left(1+\frac{M}{N^{2}}\right),
$$

with an implied constant being a function of $h$. Merging the latter bound with (4.40) indeed confirms the estimate in 4.28 for $\mathscr{M}_{2}^{0}(\alpha)$, which ends our considerations of this term.

\subsection{Large sieve inequalities for Bessel functions I}

In the previous two subsections we have established the bounds in 4.28. Since we have the split of the second main term $\mathscr{M}^{0}(\alpha)$ in 4.27 , we immediately know now that

$$
\mathscr{M}^{0}(\alpha) \ll\|\alpha\|^{2} M^{\varepsilon}\left(1+\frac{M}{N^{2}}\right) .
$$


The results of the previous two subsections however depend on the verification of Lemma 4.6, As already mentioned it is much in the style of the the large sieve inequality given in [15, Lemma 9.1]. Proving Lemma 4.6 is at the core of this section and for convenience we restate the statement of our result for the $Y$ - and the $J$-Bessel function here. Let $H$ and $T$ be as in conditions of Lemma 4.5, then with $1 \leq Z \leq T / 2$ and $\Delta \leq R \leq H$ we seek to bound

$$
\mathcal{M}_{B}(Z, R ; \nu):=\sum_{Z<t \leq 2 Z} \int_{R}^{2 R} \sum_{x \bmod t}^{*}\left|\sum_{M<n \leq 2 M} a_{n} e\left(\frac{x n}{t}\right) B_{\nu}\left(\frac{4 \pi}{t} \sqrt{\frac{y n}{N}}\right)\right|^{2} d y
$$

with

$$
\|\alpha\|^{2} N M^{\varepsilon} Z^{2}\left(1+\left(\frac{Z^{2} R}{N M}\right)^{1 / 2}\right)\left(1+|\Im \nu|^{9}\right) e^{2 \pi|\Im \nu|}
$$

valid for any $\alpha \in \mathbb{C}_{M}^{\infty}$. This provides us in fact with two large sieve inequalities, since $B_{\nu}(x)$ may represent both the $Y$ - or the $J$-Bessel function of complex index $\nu \in \mathbb{C}$ with $|\Re \nu|<1 / 2$. An important feature of this inequality is that it is uniform in the imaginary part of the index. The proof of Lemma 4.6 follows closely the argument supporting [15, Lemma 9.1, (see for statement and proof p. 555-556)] and although the strategy and outline of the following argument are similar to the proof in [15] just mentioned, we will retrace its steps here carefully to adjust it to the present situation.

Proof of Lemma 4.6. The desired upper bound in (4.43), which we aim for when estimating $\mathcal{M}_{B}(Z, R ; \nu)$ in the following, relies on two sources. In a sense the contribution of the $y$-integration in 4.42) is controlled exploiting the oscillation from the Bessel functions via integration by parts. Furthermore the oscillation of the exponential in the definition of (4.42) will be employed via a large sieve inequality to facilitate the desired bound for $\mathcal{M}_{B}(Z, R ; \nu)$. With these two intentions in mind we begin to manipulate the expression in 4.42 and first define $Q:=\left(R M / 4 Z^{2} N\right)^{1 / 2}$. Now with a change of variables in the integration of this term, we immediately obtain that

$$
\mathcal{M}_{B}(Z, R ; \nu) \ll \frac{R}{Q} \sum_{Z<t \leq 2 Z} \int_{Q}^{3 Q} \sum_{x \bmod t}^{*} \mid \sum_{M<n \leq 2 M} a_{n} e\left(\frac{x n}{t}\right) B_{\nu}\left(4 \pi q \sqrt{\frac{n}{M}}\right)^{2} d q
$$

where we let $q=\left(y M / N t^{2}\right)^{1 / 2}$. With Lemma 7.7 we separate the Bessel function into a sum of products of functions of which one part is smooth and the other one oscillates. Concretely we see that $\mathcal{M}_{B}(Z, R ; \nu)$ is bounded further by

$$
\frac{R}{Q} \sum_{ \pm} \sum_{Z<t \leq 2 Z} \int_{Q}^{3 Q} \sum_{x \bmod t}^{*}\left|\sum_{M<n \leq 2 M} A_{n} e\left(\frac{x n}{t} \pm 2 q \sqrt{\frac{n}{M}}\right) \Omega_{ \pm}\left(\nu, 2 q \sqrt{\frac{n}{M}}\right)\right|^{2} \frac{d q}{q}
$$

where $A_{n}:=a_{n}(M / n)^{1 / 4}$. For the functions $\Omega_{ \pm}$we recall the bound given in $(7.23)$. Furthermore its support is in $\left[(9 M)^{-1}, 77 M^{1+\varepsilon}\right]$ and hence we may employ Lemma 7.8 to separate variables in the real argument of $\Omega_{ \pm}$via the inverse Mellin transform. In the notation of Lemma 7.8 we obtain from it

$$
\frac{R}{Q^{2}} \sum_{ \pm} \sum_{Z<t \leq 2 Z} \int_{Q}^{3 Q} \sum_{x \bmod t}^{*}\left|\int_{(0)} \widehat{G}_{ \pm}(s) \sum_{M<n \leq 2 M} A_{n}(q, s) e\left(\frac{x n}{t} \pm 2 q \sqrt{\frac{n}{M}}\right) d s\right|^{2} d q
$$


where $A_{n}(q, s):=A_{n} \cdot\left(M / q^{2} n\right)^{s / 2}$. We will now proceed by considering the complex vector in intervals of length $\mathscr{X}=M / Q$. Introducing $\mathscr{Q}=M+m \mathscr{X}$, with $0 \leq m \leq Q$, and recalling the support of the vector $\alpha$, we may rewrite the sum over $n$ in (4.44) as

$$
\sum_{M \leq \mathscr{Q} \ll M} e\left( \pm 2 q \sqrt{\frac{\mathscr{Q}}{M}}\right) \sum_{\mathscr{Q}<n \leq \mathscr{Q}+\mathscr{X}} A_{n}(q, s) e\left(\frac{x n}{t} \pm 2 q \frac{\sqrt{n}-\sqrt{\mathscr{Q}}}{\sqrt{M}}\right) .
$$

We point out that this split is valid regardless of $Q$ being bigger than or smaller than one. Also it is worth noticing that the argument of the corresponding exponentials in the inner sum is bounded as

$$
\pm 2 q \frac{\sqrt{n}-\sqrt{\mathscr{Q}}}{\sqrt{M}} \ll 1
$$

This will allow us later to separate variables in the exponential, by means of the power series expansion for it, without increasing the estimate by more than a constant. In order to bound (4.44) further we insert a smooth function $\tau: \mathbb{R} \rightarrow \mathbb{R}_{\geq 0}$, such that $\operatorname{supp}(\tau) \subseteq[1 / 2,4]$ with the function being equal to one on $[1,3]$, to find the estimate

$$
\frac{R}{Q^{2}} \sum_{ \pm} \sum_{Z<t \leq 2 Z} \int_{\mathbb{R}} \tau\left(\frac{q}{Q}\right) \sum_{x \bmod t}^{*}\left|\int_{(0)} \widehat{G}_{ \pm}(s) \sum_{M \leq \mathscr{Q} \ll M} \ldots \sum_{\mathscr{Q}<n \leq \mathscr{Q}+\mathscr{X}} \ldots d s\right|^{2} d q .
$$

Opening the square and using the power series expansion to separate variables, then swapping the order of integration, the latter equals

$$
\begin{aligned}
& \frac{R}{Q^{2}} \sum_{ \pm} \sum_{l, k=0}^{\infty} \frac{1}{l ! k !} \sum_{Z<t \leq 2 Z} \sum_{x \bmod t}^{*} \int_{(0)} \int_{(0)} \widehat{G}_{ \pm}\left(s_{1}\right) \overline{\widehat{G}_{ \pm}\left(s_{2}\right)} \sum_{\mathscr{Q}_{1}, \mathscr{Q}_{2}} \mathrm{I}_{ \pm}^{l, k}\left(\mathscr{Q}_{1}, \mathscr{Q}_{2} ; s_{1}, s_{2}\right) . \\
& \cdot \sum_{\mathscr{Q}_{1}<n \leq \mathscr{Q}_{1}+\mathscr{X}} A_{n}^{ \pm}\left(s_{1}, l, \mathscr{Q}_{1}\right) e\left(\frac{x n}{t}\right) \sum_{\mathscr{Q}_{2}<n \leq \mathscr{Q}_{2}+\mathscr{X}} \overline{A_{n}^{ \pm}\left(s_{2}, k, \mathscr{Q}_{2}\right)} e\left(\frac{-x n}{t}\right) d s_{1} d s_{2},
\end{aligned}
$$

with

$$
A_{n}^{ \pm}\left(s, u, \mathscr{Q}_{i}\right):=A_{n}\left(\frac{M}{n}\right)^{s / 2}\left( \pm \frac{2}{\sqrt{M}}\left(\sqrt{n}-\sqrt{\mathscr{Q}_{i}}\right)\right)^{u}
$$

and where we define

$$
\mathrm{I}_{ \pm}^{l, k}\left(\mathscr{Q}_{1}, \mathscr{Q}_{2} ; s_{1}, s_{2}\right):=\int_{\mathbb{R}} \tau\left(\frac{q}{Q}\right) q^{l+k-\left(s_{1}+\overline{s_{2}}\right)} e\left( \pm \frac{2 q}{\sqrt{M}}\left(\sqrt{\mathscr{Q}_{1}}-\sqrt{\mathscr{Q}_{2}}\right)\right) d q
$$

In order to progress we need to estimate the integral 4.46. For $\mathscr{Q}_{1}=\mathscr{Q}_{2}$ we bound it trivially and obtain immediately

$$
\mathrm{I}_{ \pm}^{l, k}\left(\mathscr{Q}_{1}, \mathscr{Q}_{2} ; s_{1}, s_{2}\right) \ll Q(4 Q)^{l+k} .
$$

Provided we encounter $\mathscr{Q}_{1} \neq \mathscr{Q}_{2}$ we want to integrate by parts. We label the phase function of the exponential

$$
\mathcal{H}_{ \pm}(q):= \pm \frac{4 \pi q}{\sqrt{M}}\left(\sqrt{\mathscr{Q}_{1}}-\sqrt{\mathscr{Q}_{2}}\right)
$$


for which we quickly see that

$$
\left|\mathcal{H}_{ \pm}^{\prime}(q)\right| \geq \frac{\left|\sqrt{\mathscr{Q}_{1}}-\sqrt{\mathscr{Q}_{2}}\right|}{\sqrt{M}}, \quad \text { and trivially } \quad \mathcal{H}_{ \pm}^{(j)}(q) \ll\left(\frac{Q}{1+l+k+\left|\Im\left(s_{1}-s_{2}\right)\right|}\right)^{-j}
$$

for $j \geq 2$ and $q \in \operatorname{supp}(\tau)$. Furthermore let $\mathcal{T}_{s_{1}, s_{2}}(q):=q^{l+k-\left(s_{1}+s_{2}\right)} \tau(q / Q)$, for which we obtain easily

$$
\mathcal{T}_{s_{1}, s_{2}}^{(j)}(q) \ll(4 Q)^{l+k}\left(\frac{Q}{1+l+k+\left|\Im\left(s_{1}-s_{2}\right)\right|}\right)^{-j},
$$

for $j \geq 0$ and again for $q \in \operatorname{supp}(\tau)$. Then with [1, Lemma 8.1] we conclude the bound

$$
\mathrm{I}_{ \pm}^{l, k}\left(\mathscr{Q}_{1}, \mathscr{Q}_{2} ; s_{1}, s_{2}\right) \ll Q(4 Q)^{l+k}\left(\frac{\left|\mathscr{Q}_{1}-\mathscr{Q}_{2}\right|}{\mathscr{X}}\right)^{-2}\left(1+l^{2}+k^{2}+\left|\Im s_{1}\right|^{2}+\left|\Im s_{2}\right|^{2}\right)
$$

Combining this estimate with 4.47) we see that 4.45 ) is dominated by

$$
\begin{aligned}
& \frac{R}{Q} \sum_{ \pm} \sum_{l, k=0}^{\infty} \frac{(4 Q)^{l+k}}{l ! k !} \int_{(0)} \int_{(0)}\left|\widehat{G}_{ \pm}\left(s_{1}\right) \overline{\widehat{G}_{ \pm}\left(s_{2}\right)}\right|\left(1+l^{2}+k^{2}+\left|\Im s_{1}\right|^{2}+\left|\Im s_{2}\right|^{2}\right) . \\
& \quad \cdot \sum_{\mathscr{Q}_{1}, \mathscr{Q}_{2}}\left(1+\frac{\left|\mathscr{Q}_{1}-\mathscr{Q}_{2}\right|}{\mathscr{X}}\right)^{-2} \sum_{Z<t \leq 2 Z} \sum_{x \bmod t}^{*}\left|\sum_{\mathscr{Q}_{1}<n \leq \mathscr{Q}_{1}+\mathscr{X}} \ldots\right|\left|\sum_{\mathscr{Q}_{2}<n \leq \mathscr{Q}_{2}+\mathscr{X}} \ldots\right|\left|d s_{1} d s_{2}\right| .
\end{aligned}
$$

We want to use a large sieve inequality for additive characters. For this tool to become applicable we first employ the Cauchy Schwarz inequality in the summation over the residue classes. Now we may estimate both of the factors resulting from the application of the Cauchy Schwarz inequality using the large sieve from [14, Theorem 7.11], which gives us

$$
\begin{aligned}
& \frac{R}{Q}\left(Z^{2}+\mathscr{X}\right)\left(\log \left(3 \sqrt{2} \pi M^{1+\varepsilon}\right)\right)^{2}\left(1+|\Im \nu|^{9}\right) e^{2 \pi|\Im \nu|} . \\
& \quad \cdot \sum_{\mathscr{Q}_{1}, \mathscr{Q}_{2}}\left(1+\frac{\left|\mathscr{Q}_{1}-\mathscr{Q}_{2}\right|}{\mathscr{X}}\right)^{-2}\left(\sum_{\mathscr{Q}_{1}<n \leq \mathscr{Q}_{1}+\mathscr{X}}\left|a_{n}\right|^{2}\right)^{1 / 2}\left(\sum_{\mathscr{Q}_{2}<n \leq \mathscr{Q}_{2}+\mathscr{X}}\left|\bar{a}_{n}\right|^{2}\right)^{1 / 2},
\end{aligned}
$$

where Lemma 7.8 in particular 7.30 with $j=4$, provides an upper bound for the integrals involved and we used

$$
A_{n}^{ \pm}\left(s, u, \mathscr{Q}_{i}\right) \ll\left|a_{n}\right| \cdot(2 / Q)^{u},
$$

since we have $n \leq \mathscr{Q}_{i}+\mathscr{X}$ with $M \leq \mathscr{Q}_{i} \ll M$. It remains to estimate the sums over $\mathscr{Q}_{1}$ and $\mathscr{Q}_{2}$ in (4.49) and recover the norm of the original vector $\alpha$ in the process. For the diagonal terms $\mathscr{Q}_{1}=\mathscr{Q}_{2}$ we immediately see that this part of the sum contributes

$$
\sum_{\mathscr{Q}} \sum_{\mathscr{Q}<n \leq \mathscr{Q}+\mathscr{X}}\left|a_{n}\right|^{2}=\|\alpha\|^{2}
$$

Now we need to handle the off-diagonal terms for which $\mathscr{Q}_{1} \neq \mathscr{Q}_{2}$. First we observe that this sum does not exceed

$$
\sum_{\mathscr{Q}_{1}, \mathscr{Q}_{2}}\left(1+\frac{\left|\mathscr{Q}_{1}-\mathscr{Q}_{2}\right|}{\mathscr{X}}\right)^{-2} \sum_{i=1,2} \sum_{\mathscr{Q}_{i}<n \leq \mathscr{Q}_{i}+\mathscr{X}}\left|a_{n}\right|^{2} .
$$


Both of the resulting sums are treated by a symmetric argument. We will therefore restrict to demonstrating only one. From the definition of the $\mathscr{Q}_{i}$ 's we see that $\mathscr{Q}_{1}-\mathscr{Q}_{2} \equiv 0(\bmod \mathscr{X})$ and hence we obtain for the first sum the estimate

$$
\sum_{\mathscr{Q}_{1}} \sum_{\mathscr{Q}_{1}<n \leq \mathscr{Q}_{1}+\mathscr{X}}\left|a_{n}\right|^{2} \sum_{\mathscr{Q}_{3}=0}^{\infty} \frac{1}{1+\left(\mathscr{Q}_{3}\right)^{2}} \ll\|\alpha\|^{2},
$$

where we introduced the variable $\mathscr{Q}_{3}:=\left(\mathscr{Q}_{1}-\mathscr{Q}_{2}\right) / \mathscr{X} \in \mathbb{Z}$. Combining 4.50$)$ and the bounds derived in the style of (4.51) to control the size of the sums in 4.49 we conclude that

$$
\mathcal{M}_{B}(Z, R ; \nu) \ll \frac{R}{Q}\left(Z^{2}+\mathscr{X}\right)\left(\log \left(3 \sqrt{2} \pi M^{1+\varepsilon}\right)\right)^{2}\left(1+|\Im \nu|^{9}\right) e^{2 \pi|\Im \nu|} .
$$

Recalling the definition of $Q$ and that $\mathscr{X}=M / Q$, from the latter bound we quickly see the lemma. 


\section{Chapter 5}

\section{An asymptotic formula for the discrete spectrum}

\subsection{The main theorem}

The final goal of this thesis is to establish a large sieve estimating $\xi^{\text {disc }}(\alpha)$, as defined in 4.2 , This inequality will follow from an asymptotic expansion of the $\operatorname{sum} \xi^{\text {disc }}(\alpha)$, in a form we will establish in Chapter 6. En route of proving this result we continue to work with the asymptotic expansion given in Theorem 4.2. The asymptotic formula described in this theorem expands in fact the term $\xi(\alpha)$. Recalling its definition in 4.1 we see that this term, in addition to the desired $\xi^{\text {disc }}(\alpha)$, also features $\xi^{\text {cont }}(\alpha)$, defined in (4.3). This chapter is therefore devoted to taking care of the latter term, a task during which we are going to rely on the validity of the Riemann Hypothesis $(\mathrm{RH})$, as introduced in Section 2.5. which from now on for the rest of this thesis we assume to be true. We are going to show the following result.

Theorem 5.1. We assume the validity of RH, as in The Riemann hypothesis 2.7 (cp. Section 2.5). Just as in Theorem 4.2 let the level $N$ be an odd prime and where we set $H=327 T^{1+\delta}$ and $T=M / N>1$. Then we find for any $\alpha \in \mathbb{C}_{M}^{\infty}$ that

$$
\xi^{\text {disc }}(\alpha)=\mathscr{M}_{h \neq 0}(\alpha)+\mathcal{O}\left(\|\alpha\|_{\infty}^{2}\left(1+\frac{M}{N^{2}}\right) M^{1+\varepsilon}\right)
$$

for any $\varepsilon>0$. The main term here $\mathscr{M}_{h \neq 0}(\alpha)$ is given by

$$
\frac{1}{N} \sum_{\substack{1 \leq t \leq T \\(t, N)=1}} \frac{1}{t^{2}} \sum_{1 \leq|h| \leq 2 H} f\left(\frac{h}{H}\right) \sum_{M<m, n \leq 2 M} \bar{a}_{m} a_{n} S(h \bar{N}, m, t) S(h \bar{N}, n, t) \mathscr{I}\left(\frac{m}{N t}, \frac{n}{N t}, \frac{h}{t}\right),
$$

where in the latter term $f: \mathbb{R} \rightarrow \mathbb{R}_{\geq 0}$ may be again any smooth even function, admitting $\operatorname{supp}(f) \subseteq[-2,2]$ and that takes the value one on $[-1,1]$. We recall once more the function $\mathscr{I}$, as for instance described before in Theorem 4.2 In the following J, Y and $K$ denote as before the corresponding Bessel functions and we have for summation index $h>0$ that

$$
\mathscr{I}\left(\frac{m}{N t}, \frac{n}{N t}, \frac{h}{t}\right)=-\mathrm{i} \int_{\mathbb{R}} \frac{4 \pi^{2} h(x)}{\sinh ^{2}(\pi x)} J_{2 \mathrm{i} x}\left(\frac{4 \pi}{t} \sqrt{\frac{h m}{N}}\right) \Im\left(J_{2 \mathrm{i} x}\left(\frac{4 \pi}{t} \sqrt{\frac{h n}{N}}\right)\right) d_{\mathrm{spec}} x,
$$


and for $h<0$ we find

$$
\mathscr{I}\left(\frac{m}{N t}, \frac{n}{N t}, \frac{h}{t}\right)=\mathrm{i} \int_{\mathbb{R}} \frac{8 \pi h(x)}{\tanh (\pi x)} Y_{2 \mathrm{i} x}\left(\frac{4 \pi}{t} \sqrt{\frac{|h| \max \{m, n\}}{N}}\right) K_{2 \mathrm{i} x}\left(\frac{4 \pi}{t} \sqrt{\frac{|h| \min \{m, n\}}{N}}\right) d_{\mathrm{spec}} x .
$$

This result is a direct consequence of Theorem 4.2 and Proposition 5.2, which we will give in the next section. Before we move on to do so, we make some remarks about the asymptotic expansion given in (5.1). For technical reasons from this point onwards the vector $\alpha$ will be estimated with the infinity norm $\|\alpha\|_{\infty}$, for which we trivially have that $\|\alpha\|^{2} \leq M\|\alpha\|_{\infty}^{2}$. Thus all the previous results in terms of the 2-norm, may simply be converted into statements reflecting the infinity norm. Furthermore we point out that the main term of the asymptotic in $\sqrt[5.1)]{ }$, in contrast to the main term $\mathscr{M}(\alpha)$ in $\sqrt{4.4}$, does not contain the term with summation index $h=0$ any more. Indeed we separate the zeroth term in the $h$-summation of the main term in (4.4) from the rest and reflect this split by writing

$$
\mathscr{M}(\alpha)=\mathscr{M}_{h \neq 0}(\alpha)+\mathscr{M}_{h=0}(\alpha)
$$

where the latter term for $h=0$ is given by

$$
\frac{2}{N \pi} \sum_{\substack{1 \leq t \leq T \\(t, N)=1}} \frac{1}{t^{2}} \sum_{M<m, n \leq 2 M} \bar{a}_{m} a_{n} c_{t}(m) c_{t}(n) \int_{\mathbb{R}} h(x)\left(\frac{\sqrt{m n}}{\max \{m, n\}}\right)^{2 \mathrm{i} x} d x
$$

This follows at once from the expression given for the function $\mathscr{I}(*, *, 0)$, as for instance specified in Theorem 4.2, recalling that the cut-off function satisfies $f(0)=1$, and the fact that a Kloosterman sum with first argument equal to zero is simply a Ramanujan sum, which we denote by $c_{t}(k)$. The importance of the term $\mathscr{M}_{h=0}(\alpha)$ and its further treatment is subject to the next section.

\subsection{Matching Eisenstein terms}

As announced previously in this section we are going to investigate the role of the term $\mathscr{M}_{h=0}(\alpha)$, as part of the main term of the asymptotic formula established in Theorem 4.2. It turns out the this part of $\mathscr{M}(\alpha)$ cannot just easily be estimated to satisfactory quality for it to be absorbed by the error term in 4.4 . However we can still get rid of this term; we deal with it in the following manner.

Proposition 5.2. We suppose that RH holds, as given in The Riemann hypothesis 2.7 (cp. Section 2.5). For any $\alpha \in \mathbb{C}_{M}^{\infty}$, odd prime level $N$ and $T=M / N>1$ we find that

$$
\xi^{\mathrm{cont}}(\alpha)=\mathscr{M}_{h=0}(\alpha)+\mathcal{O}\left(\|\alpha\|_{\infty}^{2}\left(1+\frac{M}{N^{2}}\right) M^{1+\varepsilon}\right)
$$

for any small $\varepsilon>0$. The main term $\mathscr{M}_{h=0}(\alpha)$ is just as defined in 5.2 .

This proposition offers an interesting interpretation of the term $\mathscr{M}_{h=0}(\alpha)$. It tells us that, up to a for our purposes sufficiently small error, this part of the main term reflects the term $\xi^{\operatorname{cont}}(\alpha)$, on the left hand side of the asymptotic from Theorem 4.2 The term $\xi^{\operatorname{cont}}(\alpha)$ is reminiscent of the continuous spectrum of the hyperbolic Laplace operator $\Delta_{\text {Laplace. }}$. Indeed this connection may be observed by recalling the spectral decomposition of $L^{2}\left(\Gamma_{0}(N) \backslash \mathbb{H} ; \chi\right)$ for $\Delta_{\text {Laplace }}$ (cp. for instance [14. Theorem 15.5] for the principal character $\chi_{0}$ and the combination of [7, Proposition 4.1]) and [7, Proposition 4.2]) in case of the character $\chi$ being primitive.) featuring both, functions for which the hyperbolic Laplace operator has discrete and continuous spectrum. In turn this decomposition is an essential ingredient when it comes to establishing the Bruggeman-Kuznetsov formula, as in Theorem 2.2. and thus for the larger family in Theorem 2.3, giving 
rise to the term $\xi^{\text {cont }}(\alpha)$, which is introduced when we move to deal with quadratic forms as explained in Section 4.1. Thus in some sense the Bruggeman-Kuznetsov formula transfers information from the continuous spectrum from the spectral side to the arithmetics side, into the term which, by means of Proposition 5.2 we identify to be $\mathscr{M}_{h=0}(\alpha)$. We will now spend the remainder of this chapter presenting a rigorous argument in support of this connection. Indeed Proposition 5.2 is an immediate consequence of the following two Lemmata, which upon combining will at once prove (5.3).

Lemma 5.3. Under the assumption that RH holds, as given in The Riemann hypothesis 2.7 (cp. Section 2.5), we consider any odd prime level $N$ and let as usual $T=M / N>1$. Then for an arbitrary $\alpha \in \mathbb{C}_{M}^{\infty}$ we find for any small $\varepsilon>0$ that

$$
\xi^{\mathrm{cont}}(\alpha)=\mathscr{M}_{h=0}^{\infty}(\alpha)+\mathcal{O}\left(\|\alpha\|_{\infty}^{2} \frac{M^{2+\varepsilon}}{N^{2}}\right),
$$

where

$$
\mathscr{M}_{h=0}^{\infty}(\alpha):=\frac{2}{N \pi} \sum_{\substack{t=1 \\(t, N)=1}}^{\infty} \sum_{M<m, n \leq 2 M} \bar{a}_{m} a_{n} \frac{c_{t}(m) c_{t}(n)}{t^{2}} \int_{\mathbb{R}} h(x)\left(\frac{\sqrt{m n}}{\max \{m, n\}}\right)^{2 \mathrm{i} x} d x .
$$

This lemma already almost gives the statement we claimed in Proposition 5.2. What is left to fully obtain the latter result, is a minor manipulation of the main term of the asymptotic in the previous lemma, causing an acceptable alteration of the error term as well. The desired change is provided by the upcoming lemma.

Lemma 5.4. Let $T=M / N>1$, for odd prime level $N$ and $M$ the length of the interval of support of any $\alpha \in \mathbb{C}_{M}^{\infty}$. Then, for any $\varepsilon>0$, we find that

$$
\mathscr{M}_{h=0}^{\infty}(\alpha)=\mathscr{M}_{h=0}(\alpha)+\mathcal{O}\left(\|\alpha\|_{\infty}^{2} M^{1+\varepsilon}\right),
$$

where again the main term of the asymptotic is specified in (5.2).

The proof of Lemma 5.3 is long and somewhat technical, so we postpone a full account thereof to the next section. Lemma 5.4 however is rather immediate and thus we will finish this section by giving a short proof of it.

Proof of Lemma 5.4 In order to show the asymptotic expansion given in the lemma, we will estimate the contribution to $\mathscr{M}_{h=0}^{\infty}(\alpha)$ of the $t$ sum with large summation index. Indeed we separate at the parameter $T$ to obtain

$$
\mathscr{M}_{h=0}^{\infty}(\alpha)=\mathscr{M}_{h=0}(\alpha)+\frac{2}{N \pi} \sum_{\substack{t>T \\(t, N)=1}} \ldots,
$$

and begin to bound the latter term in which we encounter $t>T$. We control the sum in question directly, using the standard well known bound that for any $t, k \in \mathbb{N}$ we have

$$
\left|c_{t}(k)\right| \leq(t, k)
$$

(cp. for instance [14, (3.5), p.45]) for a Ramanujan sum. The resulting inner $m, n$-summations are seen to admit each an upper bound

$$
\sum_{M<m \leq 2 M}(t, m) \ll M \cdot d(t) \ll M \cdot t^{\varepsilon}
$$


where $d(\cdot)$ denotes the divisor function. With the latter estimate we then obtain

$$
\frac{2}{N \pi} \sum_{\substack{t>T \\(t, N)=1}} \cdots \ll \frac{\|\alpha\|_{\infty}^{2} M^{2}}{N} \sum_{\substack{t>T \\(t, N)=1}} \frac{1}{t^{2-\varepsilon}} \ll \frac{\|\alpha\|_{\infty}^{2} M^{2}}{N T^{1-\varepsilon}} \ll\|\alpha\|_{\infty}^{2} M^{1+\varepsilon},
$$

where we employ partial summation to bound the $t$ sum. The implied constant depends on the Schwartz function $h$, for which we recall its decay properties as stated in Section 2.4. These in turn guarantee absolute convergence of the involved integral over this function. Finally, using the latter estimate in 5.4, we conclude the statement of the lemma.

\subsection{An asymptotic formula for $\xi^{\operatorname{cont}}(\alpha)$}

The purpose of this section is to establish the asymptotic formula for $\xi^{\text {cont }}(\alpha)$, as given in Lemma 5.3. For convenience we recall the definition of the term we try to approximate as given in Section 4.1. Therein we introduced

$$
\xi^{\mathrm{cont}}(\alpha)=\left.\frac{1}{2 \pi \varphi(N)} \sum_{\chi \bmod N}^{+} \sum_{\mathfrak{a}} \int_{\mathbb{R}} \sum_{M<m \leq 2 M} a_{m} \tau_{\mathfrak{a}, \chi}(m, w)\right|^{2} \frac{h(w)}{\cosh (\pi w)} d w,
$$

where we recall that coherent with the presentation of the Bruggeman-Kuznetsov formula, we employ as Theorem 2.2 as the starting point of our considerations, $\tau_{\mathfrak{a}, \chi}(m, x)$ are the Fourier coefficients of the Eisenstein series at $s=1 / 2+\mathrm{i} w$, coming from its Fourier expansior ${ }^{(\mathrm{I})}$

$$
\begin{aligned}
& E_{\mathfrak{a}}(z, 1 / 2+\mathrm{i} w) \\
& \quad=\delta_{\mathfrak{a}, \infty} y^{1 / 2+\mathrm{i} w}+\tau_{\mathfrak{a}, \chi}^{0}(1 / 2+\mathrm{i} w) y^{1 / 2-\mathrm{i} w}+y^{1 / 2} \sum_{m \in \mathbb{Z} \backslash\{0\}} \tau_{\mathfrak{a}, \chi}(m, w) K_{\mathrm{i} w}(2 \pi|m| y) e(m x) .
\end{aligned}
$$

In the above we use the Kronecker-Delta, by which we mean in this context that

$$
\delta_{\mathfrak{a}, \infty}:= \begin{cases}1, & \text { if } \mathfrak{a} \sim \infty \\ 0, & \text { otherwise }\end{cases}
$$

In the summation in (5.7) we sum the Fourier coefficients against the $K$-Bessel function and an exponential. Given the description in (5.6), in order to analyse $\xi^{\mathrm{cont}}(\alpha)$ it makes sense to consider the Fourier coefficients of the Eisenstein series in explicit form and then extract from the resulting expression the appropriate main term and estimate the remainder as error term. The outcome will be summarised as Lemma 5.3 We are now going to execute the outline of the strategy of the proof.

\subsubsection{Fourier coefficients of Eisenstein series}

We will give an explicit descriptions for the Fourier coefficients of the Eisenstein series, for all possible Dirichlet characters $\chi$ of modulus $N$ and every cusp. The congruence subgroup $\Gamma_{0}(N)$ of prime level $N$ has exactly two inequivalent cusps, which are $\mathfrak{a} \sim \infty$ and $\mathfrak{a} \sim 0$ (cp. Section 2.2). We will show the following lemma.

\footnotetext{
(I) cp. for instance [14 (16.22), p.407] for the principal character $\chi_{0}$ and [7] (5.3), p.514] for weight $k=0$ together with Lemma 7.10 for primitive characters.
} 
Lemma 5.5. Let $N$ be an odd prime. For $m>0$ and $w \in \mathbb{R}$ we denote by $\tau_{\mathfrak{a}, \chi}(m, w)$ the Fourier coefficients of the Eisenstein series with Fourier expansion as given in (5.7) and we suppose that RH is true, as given in The Riemann hypothesis 2.7 (cp. Section 2.5). Then for any primitive Dirichlet character $\chi$ of modulus $N$ we have

$$
\bar{\tau}_{\infty, \chi}(m, w) \tau_{\infty, \chi}(n, w)=\frac{4 \cosh (\pi w)}{N}\left(\frac{m}{n}\right)^{\mathrm{i} w} \sum_{l, k=1}^{\infty} \frac{\bar{\chi}(l) \chi(k) c_{l}(m) c_{k}(n)}{l k}\left(\frac{k}{l}\right)^{2 \mathrm{i} w}
$$

and

$$
\bar{\tau}_{0, \chi}(m, w) \tau_{0, \chi}(n, w)=\frac{4 \cosh (\pi w)}{N}\left(\frac{n}{m}\right)^{\mathrm{i} w} \sum_{l, k=1}^{\infty} \frac{\bar{\chi}(l) \chi(k) c_{l}(m) c_{k}(n)}{l k}\left(\frac{l}{k}\right)^{2 \mathrm{i} w}
$$

where in both expressions $c_{t}(h)$ represents again a Ramanujan sum. Now, for the only non-primitive character of prime modulus $N$, the principal character $\chi_{0}$, we obtain

$$
\bar{\tau}_{\infty, \chi_{0}}(m, w) \tau_{\infty, \chi_{0}}(n, w)=4 \cosh (\pi w)\left(\frac{n}{m}\right)^{\mathrm{i} w} \sum_{\substack{l, k=1 \\ l, k=0 \bmod N}}^{\infty} \frac{c_{l}(m) c_{k}(n)}{l k}\left(\frac{l}{k}\right)^{2 \mathrm{i} w}
$$

and

$$
\bar{\tau}_{0, \chi_{0}}(m, w) \tau_{0, \chi_{0}}(n, w)=\frac{4 \cosh (\pi w)}{N}\left(\frac{n}{m}\right)^{\mathrm{i} w} \sum_{l, k=1}^{\infty} \frac{\chi_{0}(l) \chi_{0}(k) c_{l}(m) c_{k}(n)}{l k}\left(\frac{l}{k}\right)^{2 \mathrm{i} w} .
$$

As before we sum over Ramanujan sums.

Proof. We begin by showing the two expressions for the two cusps in case of any primitive character $\chi$ modulo $N$. In [7] we find explicit calculations for the Fourier coefficients of Eisenstein series. The scenario fits our purposes upon choosing the weight $k=0$. In this case in [7, (4.72)] the authors give for $w \in \mathbb{R}$ the Fourier expansion

$$
E_{\mathfrak{a}}(z, 1 / 2+\mathrm{i} w)=\delta_{\mathfrak{a}, \infty} y^{1 / 2+\mathrm{i} w}+\varphi_{\mathfrak{a}}(1 / 2+\mathrm{i} w) y^{1 / 2-\mathrm{i} w}+\sum_{m \in \mathbb{Z} \backslash\{0\}} \rho_{\mathfrak{a}}(m, w) W_{0, \mathbf{i} w}(4 \pi|m| y) e(m x)
$$

for a Eisenstein series $E_{\mathfrak{a}}$ with respect to the cusp a. The term $\varphi_{\mathfrak{a}}$ is for instance given explicitly in [7, (7.12)], however will be unimportant in our case. For a coherent presentation with the rest of the chapter we have made some minor adjustments to the notation of the presentation in 5.8. The expansion is given in terms of the Whittaker function $W_{0, i w}$, which by means of [7, (4.20) [(II)] may be written as the integral

$$
W_{0, \mathrm{i} w}(4 \pi|m| y)=\frac{e^{-2 \pi|m| y}}{\Gamma(1 / 2+\mathrm{i} w)} \int_{0}^{\infty} e^{-t}\left(t+\frac{t^{2}}{4 \pi|m| y}\right)^{\mathrm{i} w-1 / 2} d t .
$$

We adjust now the normalisation of the Fourier coefficients. Using Lemma 7.10 we may write the Fourier expansion in 5.8) in terms of the $K$-Bessel function and compare it to the expansion in (5.7) to find for $m \neq 0$ that

$$
\tau_{\mathfrak{a}, \chi}(m, w)=2|m|^{1 / 2} \rho_{\mathfrak{a}}(m, w) .
$$

\footnotetext{
(II) In [7] (4.20)] the second factor in the integrand should read $t^{\beta-\alpha-1 / 2}$; compare for instance with [10] 9.222.2].
} 
Now employing the latter renormalisation of the Fourier coefficients $\tau_{\mathfrak{a}, \chi}$, we combine [7, (6.19), (6.23) and Proposition 7.1], which provide an explicit formula for products $\bar{\rho}_{\mathfrak{a}}(m, w) \rho_{\mathfrak{a}}(n, w)$, to conclude for $m, n>0$ that

$$
\bar{\tau}_{\mathfrak{a}, \chi}(m, w) \tau_{\mathfrak{a}, \chi}(n, w)=\frac{4 \pi \bar{\lambda}_{\mathfrak{a}}(m, w) \lambda_{\mathfrak{a}}(n, w)}{N\left|\Gamma(1 / 2+\mathrm{i} w) L\left(1+2 \mathrm{i} w, \bar{\chi}_{p} \chi_{q}\right)\right|^{2}},
$$

where in the denominator of the last expression we encounter a Dirichlet $L$-function and from [7, (6.17)] we have

$$
\lambda_{\mathfrak{a}}(h, w)=\sum_{a b=h} \chi_{p}(a) \chi_{q}(b)\left(\frac{a}{b}\right)^{\mathrm{i} w} .
$$

The moduli $p$ and $q$ of the Dirichlet characters in the above are found as follows. Coherent with the formulation in [7. Proposition 7.1] we consider this for cusps $\mathfrak{a} \sim 1 / p$, where $p q=N$ with $\operatorname{gcd}(p, q)=1$. Furthermore we have the decomposition $\chi=\chi_{p} \chi_{q}$, i.e. we write the Dirichlet character $\chi$ of modulus $N$ as the product of the primitive Dirichlet characters $\chi_{p}$ and $\chi_{q}$ of moduli respectively being $p$ and $q$. As we mentioned before since $N$ is prime there are only the two cusp, which are easily seen to be $\mathfrak{a} \sim 1 \sim 0$ and $\mathfrak{a} \sim 1 / N \sim \infty$, i.e. for the pairs $(p, q)=(1, N)$ and $(N, 1)$ respectively. Since the remainder of the argument is rather similar for the two cusps, going forward we will only present the case of $\mathfrak{a} \sim \infty$. Thus for this cusp we immediately obtain from (5.9) that

$$
\begin{aligned}
& \bar{\tau}_{\infty, \chi}(m, w) \tau_{\infty, \chi}(n, w) \\
& =\frac{4 \cosh (\pi w)}{N}\left(\frac{m^{\mathrm{i} w}}{L(1+2 \mathrm{i} w, \bar{\chi})} \sum_{d \mid m} \bar{\chi}(d) d^{-2 \mathrm{i} w}\right)\left(\frac{n^{-\mathrm{i} w}}{L(1-2 \mathrm{i} w, \chi)} \sum_{d \mid n} \chi(d) d^{2 \mathrm{i} w}\right),
\end{aligned}
$$

using that $|\Gamma(1 / 2+\mathrm{i} w)|^{-2}=\cosh (\pi w) / \pi$, which is given for instance by [10, 8.332.2]. We want to analyse each of the factors, involving an $L$-function and a finite sum, further. Indeed we have that

$$
\frac{1}{L(s, \chi)} \sum_{d \mid n} \chi(d) d^{1-s}=\sum_{k=1}^{\infty} \frac{\chi(k) c_{k}(n)}{k^{s}}, \text { for } \Re(s)>1,
$$

where $c_{k}(n)$ denotes again a Ramanujan sum. This is easily observed by noticing that in the described half-plane both sides converge absolutely, recalling for the right hand side that we know for the Ramanujan sum the bound [5.5]. It is a well known fact that we may write the Ramanujan sum for every $k, n \in \mathbb{N}$ as

$$
c_{k}(n)=\sum_{d \mid(k, n)} \mu(k / d) d,
$$

(cp. [14, (3.2), p.44]), and thus employing the latter identiy we can for instance quickly move from the right hand side of (5.11) to the left, by swapping the sums, which is allowed in the region of absolute convergence. However for the identity in 5.11) to be useful in 5.10 we need it to hold for $\Re(s)=1$, as well. In order to do this we will show that

$$
\sum_{k=1}^{\infty} \frac{\chi(k) c_{k}(n)}{k^{s}} \ll(1+|\Im s|)(N \cdot n)^{\varepsilon}, \text { for } \Re(s)=1-\delta,
$$

which gives us that this sum is convergent in the half plane $\Re(s)>1-\delta$ and is furthermore also holomorphic therein. The function $L(s, \chi)$ is holomorphic for $\Re(s)>0$, and since we assume RH (see Section 2.5), we 
therefore know that the function $L(s, \chi)^{-1}$ is then holomorphic (III) in the half plane $\Re(s)>1 / 2$ and hence both sides of the equality in 5.11 describe holomorphic functions in $\Re(s)>1-\delta$, for some $\delta>0$. Thus, since 5.11 already holds for $\Re(s)>1$, as a simple consequence of the Identity Theorem we conclude that this equality must then also hold for $\Re(s)=1$. We are left to prove (5.13). Recalling that we have the representation in 5.12 and may employ this in the sum in 5.13 running over the interval $[1, \mathcal{B}]$, for some large $\mathcal{B}$. Swapping the order of summation we find

$$
\sum_{k \leq \mathcal{B}} \frac{\chi(k) c_{k}(n)}{k^{1-\delta+\mathrm{i} \Im s}}=\sum_{d \mid n} \chi(d) d^{\delta-\mathrm{i} \Im s} \sum_{k \leq \mathcal{B} / d} \frac{\chi(k) \mu(k)}{k^{1-\delta+\mathrm{i} \Im s}} .
$$

Executing the last sum, featuring as coefficients a product of a Dirichlet character and the Möbius function, by partial summation, using RH as in Version II (see Section 2.5) giving, for any $X>0$, the bound

$$
\sum_{k \leq X} \chi(k) \mu(k) \ll N^{\varepsilon} X^{1 / 2+\varepsilon},
$$

and afterwards letting $\mathcal{B} \rightarrow \infty$, we quickly arrive at (5.13). We know now that the identity in (5.11) in fact holds for $\Re(s) \geq 1$, and thus we may use it in 5.10), which immediately leads to the desired statement given in the lemma for the cusp $\mathfrak{a} \sim \infty$ for any primitive Dirichlet character $\chi$. As we mentioned before the case of the cusp $\mathfrak{a} \sim 0$ and any primitive Dirichlet character $\chi$ follows by a similar argument, which we will omit here.

We are left with treating the case of the principal character, which we are going to do by explicitly calculating the Fourier coefficients of a Eisenstein series for the principal character for each of the two cusps of $\Gamma_{0}(N)$ of prime level $N$. We begin with the cusp $\mathfrak{a} \sim 0$. The method we use is very similar to the one in [7. 7. Explicit computations on Eisenstein series] and we follow their approach closely, modifying it appropriately to fit our scenario. Indeed adapting the set up in [7, 7. Explicit computations on Eisenstein series] to the situation of the principal character and weight $k=0$, we find for $\mathfrak{a} \sim 0$ that the Eisenstein series

$$
E_{0}(z, s)=\frac{1}{2}\left(\frac{y}{N}\right)^{s} \sum_{h \in \mathbb{Z} \backslash\{0\}} \chi_{0}(h) \sum_{\substack{l \in \mathbb{Z} \\(l, h)=1}} \frac{1}{|h z+l|^{2 s}}
$$

which corresponds to [7, (7.4), p. 524]. In the above we have omitted the term $h=0$, since $\chi_{0}(0)=0$ it vanishes anyway. Furthermore the contribution of any summation index $h$ is an even function with respect to that variable. Thus we have

$$
E_{0}(z, s)=\left(\frac{y}{N}\right)^{s} \sum_{h=1}^{\infty} \frac{\chi_{0}(h)}{h^{2 s}} \sum_{a \bmod h}^{*} \sum_{m \in \mathbb{Z}} \frac{1}{\left((x+m+a / h)^{2}+y^{2}\right)^{s}}
$$

where we reparameterised the $l$-summation in (5.14) by writing $l=a+h m$, for $m \in \mathbb{Z}$ and with $a$ an invertible residue class of modulus $h$. In the latter expression we employ Poisson summation on the $m$ sum and after an appropriate change of variables we obtain

$$
E_{0}(z, s)=\left(\frac{y}{N}\right)^{s} \sum_{m \in \mathbb{Z}} e(m x) \sum_{h=1}^{\infty} \frac{\chi_{0}(h) c_{h}(m)}{h^{2 s}} \int_{\mathbb{R}} \frac{e(-n u)}{\left(u^{2}+y^{2}\right)^{s}} d u
$$

where $c_{h}(n)$ denotes as usual a Ramanujan sum. We evaluate the integral in (5.15) with [10, 3.384.9] in the case of $n \neq 0$ to write it in terms of the Whittaker function, as given for instance in [10, 9.222.2]. If we

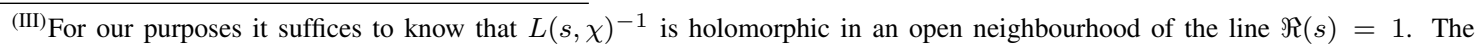
assertion that RH holds is for that in fact not necessary; the well known zero-free region in the critical strip for $L(s, \chi)$ and the fact that a possible Siegel zero does not occur at $s=1$ (see for instance [14 Theorem 5.26]) is enough.
} 
encounter $n=0$, we may employ [10, 8.381.1 and 8.384.1] to calculate the integral in terms of the Gamma function. After the computation, considering the resulting expression at $s=1 / 2+\mathrm{i} w$, we obtain from the latter immediately

$$
\begin{aligned}
E_{0}(z, 1 / 2+\mathrm{i} w)= & \left(\frac{\pi^{1 / 2} \Gamma(\mathrm{i} w)}{\Gamma(1 / 2+\mathrm{i} w) N^{1 / 2+\mathrm{i} w}} \sum_{h=1}^{\infty} \frac{\chi_{0}(h) \varphi(h)}{h^{1+2 \mathrm{i} w}}\right) y^{1 / 2-\mathrm{i} w}+ \\
& +y^{1 / 2} \sum_{m \in \mathbb{Z} \backslash\{0\}}\left(\frac{2 \pi^{1 / 2+\mathrm{i} w}|m|^{\mathrm{i} w}}{\Gamma(1 / 2+\mathrm{i} w) N^{1 / 2+\mathrm{i} w}} \sum_{h=1}^{\infty} \frac{\chi_{0}(h) c_{h}(m)}{h^{1+2 \mathrm{i} w}}\right) K_{\mathrm{i} w}(2 \pi|m| y) e(m x),
\end{aligned}
$$

where we use Lemma 7.10 to rewrite the Whittaker function in terms of the $K$-Bessel function and the well known functional equation $\Gamma(z+1)=z \Gamma(z)$ and $\Gamma(1 / 2)=\pi^{1 / 2}$ to simplify the zeroth Fourier coefficient. We recall the meromorphic continuation of the Eisenstein series (cp. again [12, Theorem 11.6, p.128] in combination with its generalisation to groups, having any finite number of cusps, as given on [12, p.295-296]) to $\mathbb{C}$ and the fact that on the line $\Re(s)=1 / 2$ one does not find any poles for it (cp. again [12, Theorem 11.8, p.130]) in combination with its generalisation to groups, having any finite number of cusp, as given on [12, p.296-298]). Now in light of (5.7), we read off directly from the last series expansion for $E_{0}(z, 1 / 2+\mathrm{i} w)$ just given the desired Fourier coefficients $\tau_{0, \chi_{0}}(m, w)$. Thus we also directly derive the formula for the product of $\bar{\tau}_{0, \chi_{0}}(m, w) \tau_{0, \chi_{0}}(n, w)$, as claimed in the lemma, employing again that from [10, 8.332.2] we know that

$$
\frac{1}{|\Gamma(1 / 2+\mathrm{i} w)|^{2}}=\frac{\cosh (\pi w)}{\pi} .
$$

The same ansatz gives in a similar way the explicit Fourier coefficients for the Eisenstein series for the principal character for the cusp $\mathfrak{a} \sim \infty$. With this remark we finish the proof.

\subsubsection{The primitive characters}

With Lemma 5.5 now in place we can now commence with establishing an asymptotic formula for $\xi^{\text {cont }}(\alpha)$. We begin by dividing $(5.6)$ into the contribution of non-principal character and the principal characters, i.e. we write

$$
\xi^{\text {cont }}(\alpha)=\xi_{\chi \neq \chi_{0}}^{\text {cont }}(\alpha)+\xi_{\chi=\chi_{0}}^{\text {cont }}(\alpha),
$$

where we postpone the treatment of the principal character to the next subsection. In the following we are going to prove that the term $\xi_{\chi \neq \chi_{0}}^{\text {cont }}(\alpha)$ in fact admits the same asymptotic expansion as the one given for $\xi^{\text {cont }}(\alpha)$ in Lemma 5.3

Proof. We begin by restating the definition of $\xi_{\chi \neq \chi_{0}}^{\text {cont }}(\alpha)$, which in light of 5.6 is

$$
\frac{1}{2 \pi \varphi(N)} \sum_{\chi \neq \chi_{0}}^{+} \sum_{\mathfrak{a}} \int_{\mathbb{R}}\left|\sum_{M<m \leq 2 M} a_{m} \tau_{\mathfrak{a}, \chi}(m, w)\right|^{2} \frac{h(w)}{\cosh (\pi w)} d w .
$$

We proceed by opening the square and then invoke Lemma 5.5 to obtain an explicit description of the Fourier coefficients at both cusps. Since the function $h$ is even by assumption, by a change of variables in the integral over one of the two resulting expressions, we may summarise the terms to

$$
\frac{4}{\pi \varphi(N) N} \int_{\mathbb{R}} h(w) \sum_{M<m, n \leq 2 M} \bar{a}_{m} a_{n} \sum_{\chi \neq \chi_{0}}^{+}\left(\frac{m}{n}\right)^{\mathrm{i} w} \sum_{l, k=1}^{\infty} \frac{\bar{\chi}(l) \chi(k) c_{l}(m) c_{k}(n)}{l k}\left(\frac{k}{l}\right)^{2 \mathrm{i} w} d w .
$$


We artificially introduce the principal character again in the character sum in the latter expression and thus we have

$$
\xi_{\chi \neq \chi_{0}}^{\mathrm{cont}}(\alpha)=\frac{4}{\pi \varphi(N) N} \int_{\mathbb{R}} \ldots \sum_{\chi \bmod N}^{+} \ldots d w-\mathscr{P}_{\chi_{0}}(\alpha)
$$

where we denote

$$
\mathscr{P}_{\chi_{0}}(\alpha):=\frac{4}{\pi \varphi(N) N} \int_{\mathbb{R}} h(w) \sum_{M<m, n \leq 2 M} \bar{a}_{m} a_{n}\left(\frac{m}{n}\right)^{\mathrm{i} w} \sum_{l, k=1}^{\infty} \frac{\chi_{0}(l) \chi_{0}(k) c_{l}(m) c_{k}(n)}{l k}\left(\frac{k}{l}\right)^{2 \mathrm{i} w} d w .
$$

Our next goal is to precisely estimate the latter term. By virtually the same argument as outlined in Subsection 5.3.1 to prove the bound given in 5.13 we show

$$
\sum_{k=1}^{\infty} \frac{\chi_{0}(k) c_{k}(m)}{k^{1 \pm 2 \mathrm{i} w}} \ll(1+|w|) d(m) N^{\varepsilon},
$$

where $d(\cdot)$ is the divisor function. With the latter bound applied to 5.18, we quickly arrive at

$$
\mathscr{P}_{\chi_{0}}(\alpha) \ll \frac{\|\alpha\|_{\infty}^{2} M^{2+\varepsilon}}{N^{2}}
$$

where the implied constant of course depends on the function $h$. Now we understand the term $\mathscr{P}_{\chi_{0}}(\alpha)$, which leaves us to analyse the second term in (5.17). Indeed we combine (5.17) with (5.19) to obtain

$$
\xi_{\chi \neq \chi_{0}}^{\text {cont }}(\alpha)=\mathscr{M}_{h=0}^{\infty}(\alpha)+\mathscr{R}(\alpha)+\mathcal{O}\left(\frac{\|\alpha\|_{\infty}^{2} M^{2+\varepsilon}}{N^{2}}\right),
$$

where

$$
\mathscr{R}(\alpha):=\frac{4}{\pi \varphi(N) N} \int_{\mathbb{R}} h(w) \sum_{M<m, n \leq 2 M} \bar{a}_{m} a_{n} \sum_{\chi \bmod N}^{+}\left(\frac{m}{n}\right)^{\mathrm{i} w} \sum_{\substack{l, k=1 \\ l \neq k}}^{\infty} \frac{\bar{\chi}(l) \chi(k) c_{l}(m) c_{k}(n)}{l k}\left(\frac{k}{l}\right)^{2 \mathrm{i} w} d w .
$$

The first main term $\mathscr{M}_{h=0}^{\infty}(\alpha)$ in 5.20 is just the diagonal term of the $l, k$ double sum in the term we have left to investigate from (5.17) and is as introduced in Lemma 5.3. This may be seen by writing the $m, n$-summation in the diagonal term of the $l, k$-double sum as

$$
\sum_{M<m \leq 2} \sum_{M<n \leq m} \cdots+\sum_{M<m \leq 2 M} \sum_{m<n \leq 2 M} \ldots,
$$

and then apply the change of variables $t \mapsto-t$ in the integration over one of the resulting two sums, recalling that the function $h$ is even by assumption.

The remainder of the proof is now concerned with estimating the term $\mathscr{R}(\alpha)$, to show that it does not contribute more than the current error term in $(5.20)$. To this end we introduce a large cut-off parameter $\mathcal{Z}>N$, for which we state an appropriate choice at a later stage. With it we split the $l, k$-double sum in the definition of $\mathscr{R}(\alpha)$ in 5.21 into

$$
\sum_{\substack{l \leq \mathcal{Z} \\ k \leq \mathcal{Z} \\ l \neq k}} \cdots+\sum_{\substack{l \leq \mathcal{Z} \\ k>\mathcal{Z}}} \cdots+\sum_{\substack{l>\mathcal{Z} \\ k \leq \mathcal{Z}}} \cdots+\sum_{\substack{l>\mathcal{Z} \\ k>\mathcal{Z} \\ l \neq k}} \cdots
$$


and name the corresponding terms $\mathscr{R}_{1}(\alpha), \mathscr{R}_{2}(\alpha), \mathscr{R}_{3}(\alpha)$ and $\mathscr{R}_{4}(\alpha)$ respectively. We will handle the situation when both $k$ and $l$ are bounded first. Observing that

$$
\sum_{\chi \bmod N}^{+} \bar{\chi}(l) \chi(k)=\frac{1}{2} \sum_{\chi \bmod N}(\bar{\chi}(l) \chi(k)+\bar{\chi}(l) \chi(-k)),
$$

we may in some sense complete the character sum to run over all Dirichlet characters of modulus $N$ and therefore are allowed to invoke orthogonality of such, to impose a congruence condition on the $k, l$ double sum. After that, estimating directly, we find that

$$
\mathscr{R}_{1}(\alpha) \ll \frac{1}{N} \sum_{M<m, n \leq 2 M}\left|\bar{a}_{m} a_{n}\right| \sum_{j=1,2} \sum_{\substack{l, k \leq \mathcal{Z} \\ l \equiv(-1) j \in \bmod N \\ l \neq k}} \frac{(l, m)(k, n)}{l k}
$$

where again we bound a Ramanujan sum with 5.5 . The implied constant depends on the function $h$. It is easy to see that

$$
\sum_{M<m \leq 2 M}\left|a_{m}\right|(h, m) \ll\|\alpha\|_{\infty} M \cdot d(h),
$$

again with $d(\cdot)$ being the divisor function. Now swapping summations in 5.23) with the latter bound in 5.24, we conclude

$$
\mathscr{R}_{1}(\alpha) \ll \frac{\|\alpha\|_{\infty}^{2} M^{2}}{N} \sum_{j=1,2} \sum_{\substack{l, k \leq \mathcal{Z} \\ l \equiv(-1) j_{\bmod } \\ l \neq k}} \frac{1}{(l k)^{1-\varepsilon}}
$$

We will treat each sum, i.e. each case of the congruence, in the previous estimate at a time. Since both sums in (5.25) are symmetric in $l$ and $k$ it suffices to consider a sum in which we investigate the behaviour for $k>l$. Thus by partial summation we quickly see that we have the bound

$$
\sum_{l \leq \mathcal{Z}} \sum_{\substack{l<k \leq \mathcal{Z} \\ k \equiv l \bmod N}} \frac{1}{(l k)^{1-\varepsilon}} \ll \frac{\mathcal{Z}^{\varepsilon}}{N}
$$

where we employ that

$$
\sum_{\substack{l<k \leq \mathcal{Z} \\ k \equiv l \bmod N}} 1 \ll \frac{\mathcal{Z}}{N}
$$

We now have to take care of the second sum in 5.25 , which considers terms with $l \equiv-k(\bmod N)$. For technical reasons it is helpful to write the summation as

$$
\sum_{l \leq \mathcal{Z}} \sum_{\substack{l<k \leq \mathcal{Z} \\ k \equiv-l \bmod N}} \frac{1}{(l k)^{1-\varepsilon}}=\sum_{l<N / 2} \sum_{\substack{N / 2<k \leq N \\ k \equiv-l \bmod N}} \cdots+\sum_{l<N / 2} \sum_{\substack{N<k \leq \mathcal{Z} \\ k \equiv-l \bmod N}} \cdots+\sum_{\substack{N / 2 \leq l \leq \mathcal{Z} \mathcal{Z} \max \{l, N\}<k \leq \mathcal{Z} \\ k \equiv-l \bmod N}} \cdots
$$

which is seen easily, considering the congruence relation satisfied by the two non-equal summation indices. As usual we interpret an empty sum as zero. We investigate each of the three sums in (5.27) after the split at a time. For the first one we obtain directly

$$
\sum_{\substack{l<N / 2 \\ l}} \sum_{\substack{N / 2<k \leq N \\ k \equiv-l \bmod N}} \frac{1}{(l k)^{1-\varepsilon}}=\sum_{l<N / 2} \frac{1}{l^{1-\varepsilon}} \sum_{1 / 2+l / N<\nu \leq 1+l / N} \frac{1}{(\nu N-l)^{1-\varepsilon}} \ll \frac{1}{N^{1-\varepsilon}},
$$


with the $\nu$-summation containing exactly the term $\nu=1$. For the second and third sum of $(5.27)$ we see by partial summation, very much in the same fashion as we obtained the bound in 5.26 , the estimates

$$
\sum_{l<N / 2} \sum_{\substack{N<k \leq \mathcal{Z} \\
k \equiv-l \bmod N}} \frac{1}{(l k)^{1-\varepsilon}} \ll \frac{\mathcal{Z}^{\varepsilon}}{N} \text { and } \sum_{\substack { N / 2 \leq l \leq \mathcal{Z} \\
\begin{subarray}{c}{\max \{l, N\}<k \leq \mathcal{Z} \\
k \equiv-l \bmod N{ N / 2 \leq l \leq \mathcal { Z } \\
\begin{subarray} { c } { \operatorname { m a x } \{ l , N \} < k \leq \mathcal { Z } \\
k \equiv - l \operatorname { m o d } N } }\end{subarray}} \frac{1}{(l k)^{1-\varepsilon}} \ll \frac{\mathcal{Z}^{\varepsilon}}{N}
$$

respectively. Hence combing the bounds for the three sums, we split into in (5.27), the original sum therein admits also the upper bound $\mathcal{Z}^{\varepsilon} / N$. Together with 5.26 , we then conclude from 5.25 , that

$$
\mathscr{R}_{1}(\alpha) \ll \frac{\|\alpha\|_{\infty}^{2} M^{2} \mathcal{Z}^{\varepsilon}}{N^{2}} .
$$

We have now taken care of the first sum of 5.22 . Next we turn our attention towards the two double sums which have one summation index bounded and one unbounded. Indeed the sums $\mathscr{R}_{2}(\alpha)$ and $\mathscr{R}_{3}(\alpha)$ can be controlled by the same technique, ultimately both having the same upper bound. Thus we will only demonstrate how to estimate one of them. For the second sum we find

$$
\mathscr{R}_{2}(\alpha) \ll \frac{1}{\varphi(N) N} \int_{\mathbb{R}}|h(w)| \sum_{M<m, n \leq 2 M}\left|\bar{a}_{m} a_{n}\right| \sum_{\chi \bmod N}^{+} \sum_{l \leq \mathcal{Z}} \frac{(l, m)}{l}\left|\sum_{k>\mathcal{Z}} \frac{c_{k}(n) \chi(k)}{k^{1-2 \mathrm{i} w}}\right| d w,
$$

again bounding the Ramanujan sum with (5.5). We are now interested into estimating the $k$-summation in the latter expression, aiming to save in the cut-off parameter $\mathcal{Z}$. First we consider the summation running in some interval $(\mathcal{Z}, \mathcal{B}]$. Rewriting the Ramanujan sum $c_{k}(n)$ using the identity in 5.12$)$ and after swapping the order of summation we obtain

$$
\sum_{\mathcal{Z}<k \leq \mathcal{B}} \frac{c_{k}(n) \chi(k)}{k^{1-2 \mathrm{i} w}}=\sum_{d \mid n} \chi(d) d^{2 \mathrm{i} w} \sum_{\mathcal{Z} / d<k \leq \mathcal{B} / d} \frac{\mu(k) \chi(k)}{k^{1-2 \mathrm{i} w}} .
$$

The last sum may now be approached by summation by parts. Assuming RH, as explained in Section 2.5 . we have the bound

$$
\sum_{k \leq X} \chi(k) \mu(k) \ll N^{\varepsilon} X^{1 / 2+\varepsilon},
$$

for any $X>0$, which allows us to conclude

$$
\sum_{\mathcal{Z} / d<k \leq \mathcal{B} / d} \frac{\mu(k) \chi(k)}{k^{1-2 \mathrm{i} w}} \ll(1+|w|) N^{\varepsilon}\left(\frac{d}{\mathcal{B}}+\frac{d}{\mathcal{Z}}\right)^{1 / 2-\varepsilon}
$$

Hence using the latter bound in 5.30 and then letting $\mathcal{B} \rightarrow \infty$, we arrive at

$$
\sum_{k>\mathcal{Z}} \frac{c_{k}(n) \chi(k)}{k^{1-2 \mathrm{i} w}} \ll \frac{(1+|w|) n^{1 / 2} N^{\varepsilon}}{\mathcal{Z}^{1 / 2-\varepsilon}} .
$$

The bound in 5.31), that we just derived, is an essential ingredient to control the sum $\mathscr{R}_{2}(\alpha)$. It ensures that, extending from (5.29), we have

$$
\mathscr{R}_{2}(\alpha) \ll \frac{\|\alpha\|_{\infty}^{2} M^{5 / 2+\varepsilon}}{N \mathcal{Z}^{1 / 2-\varepsilon}},
$$

where we used the estimate 5.24. The implied constant again depends on the function $h$. It remains to treat the sum $\mathscr{R}_{4}(\alpha)$, which is the case of both summation indices $k$ and $l$ being unbounded. Starting from 
its definition given in $(5.22)$, we see that it is again in fact enough to consider the case of $k>l$. The situation in which $l<k$ will then follow by a symmetric argument. First investigating the scenario when $l \in(\mathcal{Z}, \mathcal{B}]$, we find for such pieces of the $l, k$ double sum the bound

$$
\sum_{\mathcal{Z}<l \leq \mathcal{B}} \frac{(l, m)}{l}\left|\sum_{k>l} \frac{c_{k}(n) \chi(k)}{k^{1-2 \mathrm{i} w}}\right| \ll(1+|w|) n^{1 / 2} N^{\varepsilon} \sum_{\mathcal{Z}<l \leq \mathcal{B}} \frac{(l, m)}{l^{3 / 2-\varepsilon}},
$$

where we employ the upper bound given in (5.31) to control the $k$ sum and again 5.5 to estimate one of the Ramanujan sums. Now in the $l$-summation we observe that we have

$$
l>l^{4 \varepsilon} \mathcal{Z}^{1-4 \varepsilon},
$$

for any $\varepsilon>0$, and hence from 5.33 we estimate further by

$$
\sum_{\mathcal{Z}<l \leq \mathcal{B}} \frac{(l, m)}{l}\left|\sum_{k>l} \frac{c_{k}(n) \chi(k)}{k^{1-2 \mathrm{i} w}}\right| \ll \frac{(1+|w|) n^{1 / 2} N^{\varepsilon}}{\mathcal{Z}^{1 / 2-3 \varepsilon}} \sum_{\mathcal{Z}<l \leq \mathcal{B}} \frac{(l, m)}{l^{1+\varepsilon}} .
$$

The latter is now in fact an absolutely convergent sum and we can take $\mathcal{B} \rightarrow \infty$. With this bound for all $l>\mathcal{Z}$ in place, it is easy to see that

$$
\mathscr{R}_{4}(\alpha) \ll \frac{\|\alpha\|_{\infty}^{2} M^{5 / 2+\varepsilon}}{N \mathcal{Z}^{1 / 2-3 \varepsilon}} \sum_{l>\mathcal{Z}} \frac{d(l)}{l^{1+\varepsilon}} \ll \frac{\|\alpha\|_{\infty}^{2} M^{5 / 2+\varepsilon}}{N \mathcal{Z}^{1 / 2-\varepsilon}},
$$

again relying on 5.24 and where $d(\cdot)$ is as usual the divisor sum. Now we can put everything together. Collecting the bounds from 5.28, 5.32, which as mentioned we have also as bound for $\mathscr{R}_{3}(\alpha)$, and (5.34, we infer from 5.22) that

$$
\mathscr{R}(\alpha) \ll \frac{\|\alpha\|_{\infty}^{2} M^{2+\varepsilon}}{N}\left(\frac{\mathcal{Z}^{\varepsilon}}{N}+\frac{M^{1 / 2} \mathcal{Z}^{\varepsilon}}{\mathcal{Z}^{1 / 2}}\right) .
$$

Choosing $\mathcal{Z}=N^{2} M$ in the estimate we just obtained for $\mathscr{R}(\alpha)$, we conclude with it from 5.20 the desired asymptotic expansion

$$
\xi_{\chi \neq \neq \chi_{0}}^{\text {cont }}(\alpha)=\mathscr{M}_{h=0}^{\infty}(\alpha)+\mathcal{O}\left(\frac{\|\alpha\|_{\infty}^{2} M^{2+\varepsilon}}{N^{2}}\right),
$$

which finishes our argument.

\subsubsection{The contribution of the principal character}

In the previous subsection we split $\xi^{\text {cont }}(\alpha)$ in 5.16 into the contribution of the principal character and the primitive characters of prime modulus $N$ with correct parity. For the latter we then established in (5.35) the asymptotic expansion

$$
\xi_{\chi \neq \chi_{0}}^{\text {cont }}(\alpha)=\mathscr{M}_{h=0}^{\infty}(\alpha)+\mathcal{O}\left(\frac{\|\alpha\|_{\infty}^{2} M^{2+\varepsilon}}{N^{2}}\right) .
$$

In order to fully prove Lemma 5.3 it remains to estimate the contribution of the principal character to $\xi^{\mathrm{cont}}(\alpha)$. Indeed in light of 5.16 we will be able to conclude the result if we establish that

$$
\xi_{\chi=\chi_{0}}^{\text {cont }}(\alpha) \ll \frac{\|\alpha\|_{\infty}^{2} M^{2+\varepsilon}}{N^{2}},
$$

which is, what we are going to do now. 
Proof. First we recall the definition of the contribution of the principal character. Opening the square we find that

$$
\xi_{\chi=\chi_{0}}^{\text {cont }}(\alpha)=\frac{1}{2 \pi \varphi(N)} \sum_{\mathfrak{a}} \int_{\mathbb{R}} \sum_{M<m, n \leq 2 M} \bar{a}_{m} a_{n} \bar{\tau}_{\mathfrak{a}, \chi_{0}}(m, w) \tau_{\mathfrak{a}, \chi_{0}}(n, w) \frac{h(w)}{\cosh (\pi w)} d w .
$$

With Lemma 5.5 we may insert in the latter expression concrete formulae for the products of the Fourier coefficients of the Eisenstein series for the principal character at each of the two cusps. We obtain

$$
\begin{aligned}
& \xi_{\chi=\chi_{0}}^{\text {cont }}(\alpha) \\
& \quad=\frac{2}{\pi \varphi(N)} \int_{\mathbb{R}} h(w) \sum_{M<m, n \leq 2 M} \bar{a}_{m} a_{n}\left(\frac{n}{m}\right)^{\mathrm{i} w} \sum_{\substack{l, k=1 \\
l, k=0 \bmod N}}^{\infty} \frac{c_{l}(m) c_{k}(n)}{l k}\left(\frac{l}{k}\right)^{2 \mathrm{i} w} d w+\frac{\mathscr{P}_{\chi_{0}}(\alpha)}{2},
\end{aligned}
$$

where the contribution is, by a change of variables, just the term $\mathscr{P}_{\chi_{0}}(\alpha)$, as defined in 5.18. From 5.19) we know however already that

$$
\mathscr{P}_{\chi_{0}}(\alpha) \ll \frac{\|\alpha\|_{\infty}^{2} M^{2+\varepsilon}}{N^{2}},
$$

which is a bound of the desired quality. Hence what is left, is to estimate the contribution of the cusp $\mathfrak{a} \sim \infty$. Since the arguments, that produce suitable bounds for the contributions of the $m$ and $n$ sums in 5.36, will be essentially the same we are only going to demonstrate how to estimate

$$
\sum_{M<n \leq 2 M} a_{n} n^{\mathrm{i} w} \sum_{\substack{k=1 \\ k=0 \bmod N}}^{\infty} \frac{c_{k}(n)}{k^{1+2 \mathrm{i} w}}=\frac{1}{N^{1+2 \mathrm{i} w}} \sum_{M<n \leq 2 M} a_{n} n^{\mathrm{i} w}\left(\sum_{k \leq \mathcal{Y}} \frac{c_{k N}(n)}{k^{1+2 \mathrm{i} w}}+\sum_{k>\mathcal{Y}} \frac{c_{k N}(n)}{k^{1+2 \mathrm{i} w}}\right),
$$

where again we introduce some cut-off parameter $\mathcal{Y}$, to be specified later. We turn our attention to the sum with bounded summation index. Using the estimate (5.5) for the Ramanujan sum we obtain at once that this part is dominated by

$$
\frac{1}{N} \sum_{k \leq \mathcal{Y}} \frac{1}{k} \sum_{M<n \leq 2 M}\left|a_{n}\right|(k N, n) \ll \frac{\|\alpha\|_{\infty} M^{1+\varepsilon} \mathcal{Y}^{\varepsilon}}{N},
$$

employing again (5.24) to control the $n$-summation. For the infinite part of the $k$ sum on the right hand side in 5.37 we proceed by considering it in an interval $(\mathcal{Y}, \mathcal{B}]$ first and after inserting the expression given in 5.12) for the Ramanujan sum $c_{k N}(n)$ we quickly arrive at

$$
\frac{1}{N^{1+2 \mathrm{i} w}} \sum_{M<n \leq 2 M} a_{n} n^{\mathrm{i} w} \sum_{\mathcal{Y}<k \leq \mathcal{B}} \frac{c_{k N}(n)}{k^{1+2 \mathrm{i} w}}=\sum_{M<n \leq 2 M} a_{n} n^{\mathrm{i} w} \sum_{d \mid n} d^{-2 \mathrm{i} w} \sum_{N \mathcal{Y} / d<k \leq N \mathcal{B} / d} \frac{\mu(k)}{k^{1+2 \mathrm{i} w}} .
$$

The latter $k$-summation may now be estimated using summation by parts. Since we assume RH we may employ the bound

$$
\sum_{k \leq X} \mu(k) \ll X^{1 / 2+\varepsilon}
$$

in the process (see Section 2.5). Letting $\mathcal{B} \rightarrow \infty$ we then find

$$
\frac{1}{N^{1+2 \mathrm{i} w}} \sum_{M<n \leq 2 M} a_{n} n^{\mathrm{i} w} \sum_{k>\mathcal{Y}} \frac{c_{k N}(n)}{k^{1+2 \mathrm{i} w}} \ll \frac{(1+|w|)\|\alpha\|_{\infty} M^{3 / 2+\varepsilon} \mathcal{Y}^{\varepsilon}}{(\mathcal{Y} N)^{1 / 2}} .
$$


Now we can finally bound $\xi_{\chi=\chi_{0}}^{\text {cont }}(\alpha)$. We choose $\mathcal{Y}=M N$ and combine the bounds derived in (5.38) and (5.39) in (5.37) to conclude

$$
\sum_{M<n \leq 2 M} a_{n} n^{\mathrm{i} w} \sum_{\substack{k=1 \\ k \equiv 0 \bmod N}}^{\infty} \frac{c_{k}(n)}{k^{1+2 \mathrm{i} w}} \ll \frac{(1+|w|)\|\alpha\|_{\infty} M^{1+\varepsilon}}{N} .
$$

As explained since the two are very similar, we can now employ the latter bound to control both the $m$ and $n$-summation in 5.36. Thus with 5.40 and recalling the upper bound for $\mathscr{P}_{\chi_{0}}(\alpha)$, given in 5.19), we obtain via 5.36 , the desired estimate

$$
\xi_{\chi=\chi_{0}}^{\text {cont }}(\alpha) \ll \frac{\|\alpha\|_{\infty}^{2} M^{2+\varepsilon}}{N^{2}}
$$

which finishes the proof. 


\section{Chapter 6}

\section{A large sieve for Maaß cusp forms}

\subsection{The main theorem}

In this chapter we are finally going to present the main result. We derive an asymptotic formula for $\xi^{\text {disc }}(\alpha)$, with a cut off parameter $X$ that upon varying will allow us to reduce the number of terms in the main term while increasing the error term and vice versa. From our result we will also deduce an upper bound for the whole main term and thus obtain a large sieve inequality for Maßß cusp forms, estimating

$$
\xi^{\text {disc }}(\alpha)=\frac{1}{\varphi(N) N} \sum_{\chi \bmod N}^{+} \sum_{u \in \mathcal{U}_{\chi}^{\text {Maa }}}\left|\sum_{M<m \leq 2 M} a_{m} \rho_{u}^{*}(m)\right|^{2} h\left(t_{u}\right),
$$

just as introduced in (4.2), but where we choose the normalisation for the Fourier coefficients given by

$$
\rho_{u}^{*}(m):=\rho_{u}(m)\left(\frac{2 N}{\cosh \left(\pi t_{u}\right)}\right)^{1 / 2} .
$$

With this adjustment of the Fourier coefficients $\rho_{u}^{*}(m)$ of Maaß cusp forms we expect them to be bounded on average by a constant. This estimate for $\xi^{\operatorname{disc}}(\alpha)$ is the main result of this thesis. Before however we proceed to stating the results just mentioned in more detail, we have to introduce some notation. For the purpose of estimating some or even all of the terms of the main term $\mathscr{M}_{h \neq 0}(\alpha)$, as stated in Theorem 5.1. it is useful to investigate what happens when the $h$-summation therein runs in an interval. Hence for $1 \leq X_{1} \leq X_{2} \leq 2 H$, with $H=327(M / N)^{1+\delta}$, for an odd prime $N$ and $M>N$ as in Theorem 4.2, we define the function $\Delta_{X_{1}}^{X_{2}}(\alpha)$ for any choice of $\alpha \in \mathbb{C}_{M}^{\infty}$ by

$$
\frac{1}{N} \sum_{\substack{1 \leq t \leq T \\(t, N)=1}} \frac{1}{t^{2}} \sum_{X_{1} \leq|h| \leq X_{2}} f\left(\frac{h}{H}\right) \sum_{M<m, n \leq 2 M} \bar{a}_{m} a_{n} S(h \bar{N}, m, t) S(h \bar{N}, n, t) \mathscr{I}\left(\frac{m}{N t}, \frac{n}{N t}, \frac{h}{t}\right),
$$

where we recall that the function $f: \mathbb{R} \rightarrow \mathbb{R}_{\geq 0}$ is smooth, even, admits $\operatorname{supp}(f) \subseteq[-2,2]$, and takes the value one throughout the interval $[-1,1]$. The remaining objects in the definition of $\Delta_{X_{1}}^{X_{2}}(\alpha)$ are understood to be coherent with the ones present in the main term of the asymptotic formula given in Theorem 5.1. With this quadratic form in place we are now in a position to state the main theorem of this chapter. 
Theorem 6.1. Assume the validity of RH, as in The Riemann hypothesis 2.7 (cp. Section 2.5). Consider any $\alpha \in \mathbb{C}_{M}^{\infty}$. For an odd prime level $N$ we choose $T=M / N>1$ and let $X \in[1, T] \cap \mathbb{N}$. Then we find

$$
\xi^{\text {disc }}(\alpha)=\mathscr{M}_{X}(\alpha)+\mathcal{O}\left(\|\alpha\|_{\infty}^{2}\left(\left(\frac{M}{N X}\right)^{1 / 2}+\frac{M}{N^{2}}\right) M^{1+\varepsilon}\right)
$$

with an implied constant depending on the choice of the Schwartz function h. The main term $\mathscr{M}_{X}(\alpha)$ is given by

$$
\frac{1}{N} \sum_{\substack{1 \leq t \leq T \\(t, N)=1}} \frac{1}{t^{2}} \sum_{1 \leq|h|<X} \sum_{M<m, n \leq 2 M} \bar{a}_{m} a_{n} S(h \bar{N}, m, t) S(h \bar{N}, n, t) \mathscr{I}\left(\frac{m}{N t}, \frac{n}{N t}, \frac{h}{t}\right) .
$$

In order to give a complete description we recall the definition of the function $\mathscr{I}$. If we sum in the above main term over positive $h$ we have

$$
\mathscr{I}\left(\frac{m}{N t}, \frac{n}{N t}, \frac{h}{t}\right)=-\mathrm{i} \int_{\mathbb{R}} \frac{4 \pi^{2} h(x)}{\sinh ^{2}(\pi x)} J_{2 \mathrm{i} x}\left(\frac{4 \pi}{t} \sqrt{\frac{h m}{N}}\right) \Im\left(J_{2 \mathrm{i} x}\left(\frac{4 \pi}{t} \sqrt{\frac{h n}{N}}\right)\right) d_{\mathrm{spec}} x,
$$

whereas if the h-summation takes negative values the function is

$$
\mathscr{I}\left(\frac{m}{N t}, \frac{n}{N t}, \frac{h}{t}\right)=\mathrm{i} \int_{\mathbb{R}} \frac{8 \pi h(x)}{\tanh (\pi x)} Y_{2 \mathrm{i} x}\left(\frac{4 \pi}{t} \sqrt{\frac{|h| \max \{m, n\}}{N}}\right) K_{2 \mathrm{i} x}\left(\frac{4 \pi}{t} \sqrt{\frac{|h| \min \{m, n\}}{N}}\right) d_{\mathrm{spec}} x .
$$

By J, $Y$ and $K$ we denote, coherent with the previous occurrences of $\mathscr{I}$, the corresponding Bessel functions.

The theorem just stated is the equivalent of [15. Theorem 1.1] in the context of Maaß cusp forms, and the asymptotic formula shares with the one in [15, Theorem 1.1] similar features, which we are going to discuss in more detail in the following. First of all we observe that the asymptotic formula in 6.2 comprises an interesting trade off between the number of terms of the main term $\mathscr{M}_{X}(\alpha)$ and the error term. As the parameter $X$ is chosen larger and larger, we increase the number of terms in the main term. The reward is a smaller error. Vice versa we observe that for having a main term with fewer terms, i.e. choosing $X$ closer to one, we have to pay the price by accepting a larger error term.

Furthermore we point out that Theorem 6.1 for the choice $X=[T]$ essentially just restates Theorem 5.1, however with the smaller $T-1$ as an upper bound for the interval over which we consider the $h$-summation. The most interesting observation however is probably the statement that transpires for the choice $X=1$. This means that we estimate the whole main term $\mathscr{M}_{h \neq 0}(\alpha)$ essentially as stated in Theorem 5.1, yet with the slightly revised choice $T-1$ as the upper bound for the $h$-summation, which becomes clearer after pointing our that we have roughly

$$
\mathscr{M}_{h \neq 0}(\alpha) \approx \mathscr{M}_{X}(\alpha)+\Delta_{X}^{T}(\alpha),
$$

up to an error of the same size as the one in Theorem 5.1. We will provide more details justifying this in due course. Now from the definition in 6.3 it is clear that $\mathscr{M}_{1}(\alpha)$ does not contain any terms and the error term in 6.2 will result from estimating $\Delta_{X}^{T}(\alpha)$, which in this scenario is $\Delta_{1}^{T}(\alpha) \approx \mathscr{M}_{h \neq 0}(\alpha)$. Hence estimating the whole main term, we can deduce a large sieve inequality for Maaß cusp forms, very much in a similar way as the equivalent result [15, Corollary 12.1] is derived in the case of holomorphic modular forms, and so by choosing the cut-off parameter as $X=1$ from Theorem 6.1 we arrive immediately at the estimate

$$
\sum_{\chi \bmod N}^{+} \sum_{u \in \mathcal{U}_{\chi}^{\text {Maав }}}\left|\sum_{M<m \leq 2 M} a_{m} \rho_{u}^{*}(m)\right|^{2} h\left(t_{u}\right) \ll\|\alpha\|_{\infty}^{2}\left(\left(N^{3} M\right)^{1 / 2}+M\right) M^{1+\varepsilon},
$$


which holds for every $\alpha \in \mathbb{C}_{M}^{\infty}$ and odd prime level $N$ and of course under the assumption that RH holds true. We summarise this inequality as the main result of this thesis in Theorem 1.1. It is a large sieve inequality for Maaß cusp forms taken from the family

$$
\mathcal{U}^{\mathrm{Maa} \beta}=\bigcup_{\substack{\chi \bmod N \\ \chi(-1)=1}} \mathcal{U}_{\chi}^{\mathrm{Maa} \beta}
$$

Here, for the Hecke congruence subgroup $\Gamma_{0}(N)$ of odd prime level $N$, each of $\mathcal{U}_{\chi}^{\text {Maaß }}$ represents an orthonormal basis of the space $\mathcal{C}\left(\Gamma_{0}(N) \backslash \mathbb{H} ; \chi\right)$. For a detailed discussion of this result we refer the reader to the introduction of this thesis. We will now proceed by giving a proof of Theorem 6.1 .

\subsection{Proof of the main theorem}

In this section we are going to present a proof justifying Theorem 6.1, which is in some sense a modified version of Theorem 5.1. The overall strategy of the following proof is based on the argument and ansatz establishing [15, Theorem 1.1, (for statement see p. 543 and for the proof p.557-561)] and we follow their approach and outline to some extent.

Proof. We make the same assumptions as in Theorem 5.1. Now the overall strategy is as follows: First we are going to establish a slightly stronger version of Theorem 5.1 for $T-1$ limiting the interval of the $|h|$-summation. To this end we define $T^{*}$ to be $T$, in case of $T \notin \mathbb{N}$ and to be $T+1$, provided $T \in \mathbb{N}$, i.e. $T^{*} \asymp T$, and observe that

$$
\mathscr{M}_{h \neq 0}(\alpha)=\mathscr{M}_{T^{*}}(\alpha)+\Delta_{T^{*}}^{2 H}(\alpha),
$$

where the term $\mathscr{M}_{T^{*}}(\alpha)$ is defined coherent with the previous notation introduced in 6.3 . It will turn out that the part of the main term for which $T^{*} \leq|h| \leq 2 H$ does not contribute more than the already existing error term in Theorem 4.2. Indeed we have that

$$
\Delta_{T^{*}}^{2 H}(\alpha) \ll\|\alpha\|^{2}\left(1+\frac{M}{N^{2}}\right) M^{\varepsilon} .
$$

Furthermore for a cut off parameter $X \in[1, T] \cap \mathbb{N}$, we observe that

$$
\mathscr{M}_{T^{*}}(\alpha)=\mathscr{M}_{X}(\alpha)+\Delta_{X}^{T}(\alpha)
$$

and as a result we deduce directly from Theorem 5.1 that

$$
\xi^{\text {disc }}(\alpha)=\mathscr{M}_{X}(\alpha)+\Delta_{X}^{T}(\alpha)+\mathcal{O}\left(\|\alpha\|_{\infty}^{2}\left(1+\frac{M}{N^{2}}\right) M^{1+\varepsilon}\right) .
$$

At this stage we are now very close to concluding the desired result. We point out that the cut-off function satisfies $f(x)=1$, for $-1 \leq x \leq 1$, which is always the case in the main term $\mathscr{M}_{X}(\alpha)$ in this context, since $X \leq T<327 T^{1+\delta}=H$. It remains to gain some understanding of the size to the term $\Delta_{X}^{T}(\alpha)$, which is provided by the next lemma.

Lemma 6.2. Let the quadratic form $\Delta_{X}^{T}(\alpha)$ be defined coherently with the definition in $(6.1)$, for $T=$ $M / N>1$, where $N$ is an odd prime, and for any $X \in[1, T] \cap \mathbb{N}$. Then for every choice of $\alpha \in \mathbb{C}_{M}^{\infty}$ we obtain

$$
\Delta_{X}^{T}(\alpha) \ll\|\alpha\|^{2}\left(\left(\frac{M}{N X}\right)^{1 / 2}+\frac{M}{N^{2}}\right) M^{\varepsilon},
$$

where the implied constant of this bound is a function of the Schwartz function h. 
Now, if we estimate in 6.6 the norm of the vector trivially via

$$
\|\alpha\|^{2} \ll M\|\alpha\|_{\infty}^{2},
$$

the resulting bound for $\Delta_{X}^{T}(\alpha)$ clearly always supersedes the error term in 6.5 . For this revised estimate derived from Lemma 6.2 we highlight the fact that as $X$ increases the size of the bound decreases, with the smallest possible one being of the same magnitude as the error in the asymptotic expansion in 6.5. Thus with Lemma 6.2, in which we choose to estimate the vector using 6.7, applied to 6.5), we conclude Theorem 6.1.

For a complete detailed argument supporting the main result, we have to justify Lemma 6.2 and the inequality (6.4). The latter however holds true by a slightly simpler version of the argument supporting the Lemma 6.2 The structure is very much similar in shape and applies the same intermediate results as tools. We will therefore restrict to providing a full account of the proof of Lemma 6.2 only, and omit a detailed discussion of an argument for 6.47. Establishing Lemma 6.2 is the subject of the next section.

\subsection{The size of $\Delta_{X}^{T}(\alpha)$}

In this section we will prove Lemma 6.2 We recall the definition for $\Delta_{X}^{T}(\alpha)$ as given in 6.1 and therefore want to show

$$
\Delta_{X}^{T}(\alpha) \ll\|\alpha\|^{2}\left(\left(\frac{M}{N X}\right)^{1 / 2}+\frac{M}{N^{2}}\right) M^{\varepsilon},
$$

where we impose the same conditions as in Theorem 4.2 In the proof we are going to give in this section, we will follow the same overall strategy, ansatz and outline as used in in [15] to show that estimating parts of the main term of the asymptotic expansion from [15, Theorem 10.1] causes an error term of the size present in the asymptotic formula in [15, Theorem 1.1].

Proof. Again just as at the end of the previous section we highlight the fact the the cut-off function admits $f(h / H)=1$, for every $h \in(-T, T)$, and thus in the following we always replace it by the constant function begin one in $\Delta_{X}^{T}(\alpha)$. Via the function $\mathscr{I}$ we see that producing a good estimate depends heavily on understanding the various Bessel functions involved. Thus we will study some large sieve estimates for the $J, Y$ and $K$-Bessel functions in intervals first, i.e. we restrict to the summations involved running in $t \asymp Z$ and $h \asymp R$. We have the following two lemmata.

Lemma 6.3. Let $\nu \in \mathbb{C}$, the level $N$ be an odd prime and consider $Z, R \geq 1$. For any $\alpha \in \mathbb{C}_{M}^{\infty}$ define

$$
\mathcal{N}_{1}(Z, R ; \nu):=\sum_{\substack{Z<t \leq 4 Z \\(t, N)=1}} \sum_{R<h \leq 2 R}\left|\sum_{M<n \leq 2 M} a_{n} S( \pm h \bar{N}, n, t)\left(\frac{t^{2} N}{h n}\right)^{\nu / 2}\right|^{2} .
$$

Then we obtain the bound

$$
\mathcal{N}_{1}(Z, R ; \nu) \ll\|\alpha\|^{2}\left(\frac{Z^{2} N}{R M}\right)^{\Re \nu}\left(R Z^{2}+R M+Z^{3}+Z M\right) .
$$

The bound for $\mathcal{N}_{1}(Z, R ; \nu)$ exploits random sign changes of the Kloosterman sum as $h$ and $t$ run through the indicated intervals to obtain some saving. This result is somehow connected to the $K$-Bessel function, which we will discuss in more detail in Section 6.4. Furthermore for the $J$ - and $Y$-Bessel function we have the following large sieve. 
Lemma 6.4. Let $N$ be an odd prime and $1 \leq Z \leq T$ and $1 \leq R \leq H$, where $T=M / N>1$ and $H=327 T^{1+\delta}$ are as in Theorem 4.2 For any $\alpha \in \mathbb{C}_{M}^{\infty}$ define

$$
\mathcal{N}_{2}(Z, R ; \nu):=\sum_{\substack{Z<t \leq 2 Z \\(t, N)=1}} \sum_{R<h \leq 2 R}\left|\sum_{M<n \leq 2 M} a_{n} S( \pm h \bar{N}, n, t) B_{\nu}\left(\frac{4 \pi}{t} \sqrt{\frac{h n}{N}}\right)\right|^{2}
$$

Then we have

$$
\mathcal{N}_{2}(Z, R ; \nu) \ll\|\alpha\|^{2} N Z^{2}\left(1+\frac{M}{N^{2}}+\left(\frac{M}{R N}\right)^{1 / 2}\right) M^{\varepsilon} e^{3 \pi|\Im \nu|},
$$

The function $B_{\nu}(x)$ denotes either the $Y$ - or the J-Bessel function with index $\nu \in i \mathbb{R}$ and of argument $x \in \mathbb{R}_{>0}$.

In addition to the sign changes of the Kloosterman sum the latter estimate exploits also oscillation of the Bessel functions to get a saving. For the above result we remark that Lemma 6.4 treats a scenario in a similar style as [15, Proposition 12.1]. Both Lemmata just introduced above, will play an integral part in proving Lemma 6.2. For now we postpone their proofs to Section 6.4 and proceed with our investigation of $\Delta_{X}^{T}(\alpha)$. We distinguish the sign of the summation variable $h$ and establish the bound given in 6.6 for each of the two cases separately. We write

$$
\Delta_{X}^{T}(\alpha):=\Delta_{X}^{+}(\alpha)+\Delta_{X}^{-}(\alpha)
$$

where the superscript indicates naturally the sign of the summation variable $h$ and start to treat the part for negative sign first, which is mildly more complicated than for the positive sign. We recall $\Delta_{X}^{-}(\alpha)$ is given by

$$
\begin{aligned}
& \frac{8 \mathrm{i}}{\pi N} \int_{\mathbb{R}} h(w) w \sum_{X \leq h \leq T} \sum_{\substack{1 \leq t \leq T \\
(t, N)=1}} \frac{1}{t^{2}} \sum_{M<m, n \leq 2 M} \bar{a}_{m} a_{n} S(-h \bar{N}, m, t) . \\
& \cdot S(-h \bar{N}, n, t) Y_{2 \mathrm{i} w}\left(\frac{4 \pi}{t} \sqrt{\frac{h \max \{m, n\}}{N}}\right) K_{2 \mathrm{i} w}\left(\frac{4 \pi}{t} \sqrt{\frac{h \min \{m, n\}}{N}}\right) d w .
\end{aligned}
$$

Just as in Subsection 4.6.1 we would like to get rid of the max / min functions in the arguments of the Bessel functions first. Thus we recall the function $\kappa$ as introduced in (4.32) to assist with this task and as before we separate variables in the argument of this function using the inverse Fourier transform, at the cost of an epsilon power of $M$ (cp. 4.33). At this point we split the $t$-summation in 6.9 into

$$
\sum_{X \leq h \leq T} \sum_{\substack{1 \leq t<\sqrt{T h} \\(t, N)=1}} \cdots+\sum_{X \leq h \leq T} \sum_{\substack{T, 1 \\(t, N)=1 \leq T}} \cdots
$$

which allows for more flexibility when estimating further by taking into account whether $t$ is somewhat small or large. Next we replace the $K$-Bessel functions by their inverse Mellin transforms using

$$
K_{2 \mathrm{i} w}(x)=\frac{1}{2 \pi \mathrm{i}} \int_{\left(\sigma_{j}\right)} 2^{s_{j}-2} \Gamma\left(\frac{s_{j}-2 \mathrm{i} w}{2}\right) \Gamma\left(\frac{s_{j}+2 \mathrm{i} w}{2}\right) x^{-s_{j}} d s_{j},
$$

as stated for instance in (7.31). Then Lemma 7.9 tells us that the integral in 6.10 converges absolutely for $\sigma_{j}>0$. For small $t$ a convenient choice is $\sigma_{1}=3 / 2$, whereas for large $t$ taking $\sigma_{2}=\varepsilon$ is more useful. 
After an application of the Cauchy Schwarz inequality we see that is suffices to understand

$$
\begin{aligned}
& \sum_{j=1,2} \int_{\mathbb{R}}|h(w) w| \int_{\mathbb{R}}|\hat{\kappa}(l)| \cdot \\
& \quad \int_{\left(\sigma_{j}\right)}\left|\Gamma\left(\frac{s_{j}-2 \mathrm{i} w}{2}\right) \Gamma\left(\frac{s_{j}+2 \mathrm{i} w}{2}\right)\right| \mathcal{Y}_{\text {sum }}(\alpha ; w, l)^{1 / 2} \mathcal{K}_{j}^{\mathrm{sum}}\left(\alpha ; s_{j}, l\right)^{1 / 2}\left|d s_{j}\right| d l d w,
\end{aligned}
$$

in order to control $\Delta_{X}^{-}(\alpha)$. Here we define

$$
\mathcal{Y}_{\text {sum }}(\alpha ; w, l):=\frac{1}{N} \sum_{X \leq h \leq T} \sum_{\substack{1 \leq t \leq T \\(t, N)=1}} \frac{1}{t^{2}}\left|\sum_{M<m \leq 2 M} \bar{b}_{m}(l) S(-h \bar{N}, m, t) Y_{2 \mathrm{i} w}\left(\frac{4 \pi}{t} \sqrt{\frac{h m}{N}}\right)\right|^{2},
$$

where by positivity we extended the $t$-summation to the full range, and also

$$
\mathcal{K}_{1}^{\operatorname{sum}}\left(\alpha ; s_{1}, l\right):=\frac{1}{N} \sum_{X \leq h \leq T} \sum_{\substack{1 \leq t<\sqrt{T h} \\(t, N)=1}} \frac{1}{t^{2}}\left|\sum_{M<n \leq 2 M} b_{n}(l) S(-h \bar{N}, n, t)\left(\frac{4 \pi}{t} \sqrt{\frac{h m}{N}}\right)^{-s_{1}}\right|^{2}
$$

and

$$
\mathcal{K}_{2}^{\text {sum }}\left(\alpha ; s_{2}, l\right):=\frac{1}{N} \sum_{X \leq h \leq T} \sum_{\substack{\sqrt{T h} \leq t \leq T \\(t, N)=1}} \frac{1}{t^{2}}\left|\sum_{M<n \leq 2 M} b_{n}(l) S(-h \bar{N}, n, t)\left(\frac{4 \pi}{t} \sqrt{\frac{h m}{N}}\right)^{-s_{2}}\right|^{2}
$$

where again in all three cases we write $b_{n}(l):=a_{n} e(\ln / M)$ and $\hat{\kappa}$ for the Fourier transform of $\kappa$. Estimating the latter two sums appropriately, provides more of a challenge and so we deal with it first. In both of $\mathcal{K}_{j}^{\mathrm{sum}}\left(\alpha ; s_{j}, l\right)$ we will continue by braking down the sums even further into appropriate intervals and employ the bounds already introduced for those. For small values of $t$ we find that

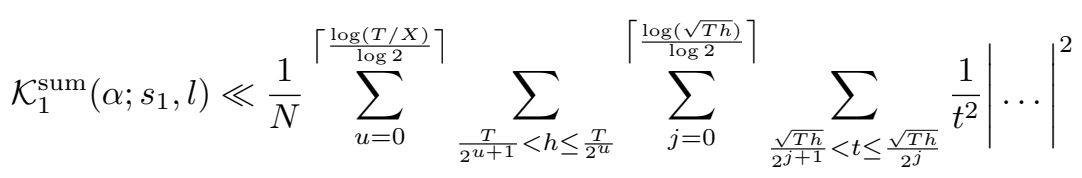

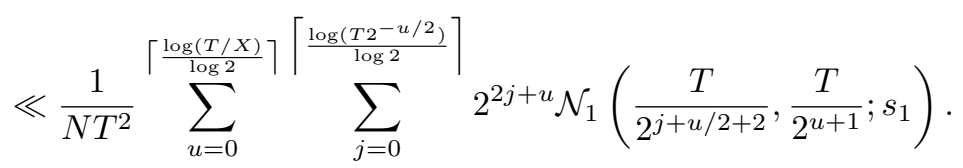

We recall that in the present situation we chose the contour in 6.10 to be $\sigma_{1}=3 / 2$ and thus with Lemma 6.3 we observe that the latter does not exceed

$$
\|\alpha\|^{2} \sum_{u=0}^{\left\lceil\frac{\log (T / X)}{\log 2}\right.} \sum_{j=0}^{\infty} 2^{u-j}\left(\frac{M}{2^{2(j+u)} N^{2}}+\frac{1}{2^{u}}+\frac{M}{2^{3 j+3 u / 2} N^{2}}+\frac{1}{2^{j+u / 2}}\right),
$$

where we invoke that $T=M / N$. From the previous bound we then deduce directly that

$$
\mathcal{K}_{1}^{\text {sum }}\left(\alpha ; s_{1}, l\right) \ll\|\alpha\|^{2}\left(\left(\frac{M}{N X}\right)^{1 / 2}+\frac{M}{N^{2}}\right) M^{\varepsilon},
$$


noticing that for $1 \leq X \leq T$ we have $M / N X \geq 1$. Now we turn our attention towards the situation for large values of $t$. We see that

$$
\begin{aligned}
\mathcal{K}_{2}^{\text {sum }}\left(\alpha ; s_{2}, l\right) & \ll \frac{1}{N} \sum_{u=0}^{\left\lceil\frac{\log (T / X)}{\log 2}\right\rceil} \sum_{\frac{T}{2^{u+1}}<h \leq \frac{T}{2^{u}}} \sum_{j=0}^{\left\lceil\frac{\log (\sqrt{T / h})}{\log 2}\right\rceil} \sum_{\frac{T}{2^{j+1}}<t \leq \frac{T}{2^{j}}} \frac{1}{t^{2}}|\ldots|^{2} \\
& \ll \frac{1}{N T^{2}} \sum_{u=0}^{\left\lceil\frac{\log (T / X)}{\log 2}\right\rceil\left\lceil\frac{u+1}{2}\right\rceil} \sum_{j=0}^{2 j} 2^{2 j}\left(\frac{T}{2^{j+2}}, \frac{T}{2^{u+1}} ; s_{2}\right) .
\end{aligned}
$$

For those values of $t$ we remark that the contour in 6.10$)$ was chosen to be $\sigma_{2}=\varepsilon$. As before we apply Lemma 6.3 and upon using once again that $T=M / N$ we therefore bound the previous expression by

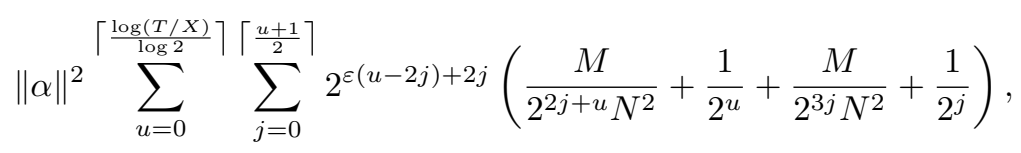

which, after estimating the contribution from the $j$-summation, is dominated by

$$
\|\alpha\|^{2} \sum_{u=0}^{\left\lceil\frac{\log (T / X)}{\log 2}\right\rceil}\left(\frac{2^{u(\varepsilon-1)} M}{N^{2}}+(u+1)+\frac{2^{\varepsilon u} M}{N^{2}}+(u+1) 2^{u / 2}\right) .
$$

The latter estimate provides us easily with the desired

$$
\mathcal{K}_{2}^{\text {sum }}\left(\alpha ; s_{2}, l\right) \ll\|\alpha\|^{2}\left(\left(\frac{M}{N X}\right)^{1 / 2}+\frac{M}{N^{2}}\right) M^{\varepsilon},
$$

where we again tidy up the bound slightly, using that the term involving $X$ always exceeds one. Now it remains to bound the sum $\mathcal{Y}_{\text {sum }}(\alpha ; w, l)$ appropriately. We proceed with a similar strategy as before. Summing intervals we see directly that

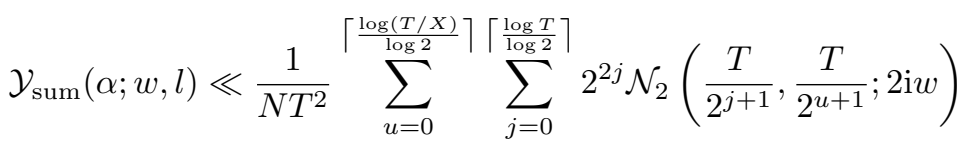

$$
\begin{aligned}
& \ll\|\alpha\|^{2} e^{6 \pi|w|} M^{\varepsilon} \sum_{u=0}^{\left\lceil\frac{\log (T / X)}{\log 2}\right\rceil}\left(1+\frac{M}{N^{2}}+2^{u / 2}\right),
\end{aligned}
$$

where the latter bound is a consequence of Lemma 6.4 Estimating the $u$-summation immediately yields the upper bound

$$
\|\alpha\|^{2} e^{6 \pi|w|}\left(\left(\frac{M}{N X}\right)^{1 / 2}+\frac{M}{N^{2}}\right) M^{\varepsilon} .
$$

Combining this bound for $\mathcal{Y}_{\text {sum }}(\alpha ; w, l)$ with the previous estimates 6.12 and 6.13 in 6.11, leads us quickly to

$$
\Delta_{X}^{-}(\alpha) \ll\|\alpha\|^{2}\left(\left(\frac{M}{N X}\right)^{1 / 2}+\frac{M}{N^{2}}\right) M^{\varepsilon},
$$


with the implied constant being dependent on the function $h$. In order to ensure convergence of all the integrals involved when we bound (6.11), we recall Lemma 7.9, the bound (4.33) to control the inverse Fourier transform of $\kappa$ and once again the fact, that we assume the function $h$ to satisfy decay properties as stated in Section 2.4. This finishes our treatment of the term $\Delta_{X}^{-}(\alpha)$, which in order to show Lemma 6.2 , leaves us to handle $\Delta_{X}^{+}(\alpha)$. We recall that the latter is given by

$$
\begin{aligned}
-\frac{4 \mathrm{i}}{N} \int_{\mathbb{R}} \frac{h(w) w}{\sinh (\pi w) \cosh (\pi w)} \sum_{X \leq h \leq T} \sum_{\substack{1 \leq t \leq T \\
(t, N)=1}} \frac{1}{t^{2}} \sum_{M<m, n \leq 2 M} \bar{a}_{m} a_{n} S(h \bar{N}, m, t) . \\
\cdot S(h \bar{N}, n, t) J_{2 \mathrm{i} w}\left(\frac{4 \pi}{t} \sqrt{\frac{h m}{N}}\right) \Im\left(J_{2 \mathrm{i} w}\left(\frac{4 \pi}{t} \sqrt{\frac{h n}{N}}\right)\right) d w .
\end{aligned}
$$

After an application of the Cauchy Schwarz inequality we realise that in order to bound the latter expression it suffices to be able to bound sums of the type

$$
\sum_{X \leq h \leq T} \sum_{\substack{1 \leq t \leq T \\(t, N)=1}} \frac{1}{t^{2}}\left|\sum_{M<n \leq 2 M} a_{n} S(h \bar{N}, n, t) J_{2 \mathrm{i} w}\left(\frac{4 \pi}{t} \sqrt{\frac{h n}{N}}\right)\right|^{2}
$$

well enough and uniformly in the index of the Bessel function. However we have estimated a similar expression when we dealt with $\mathcal{Y}_{\text {sum }}(\alpha ; w, l)$ and with essentially the same argument as for this sum, employing again Lemma 6.4, we then quickly deduce that

$$
\Delta_{X}^{+}(\alpha) \ll\|\alpha\|^{2}\left(\left(\frac{M}{N X}\right)^{1 / 2}+\frac{M}{N^{2}}\right) M^{\varepsilon},
$$

again with an absolutely convergent integral (see again Section 2.4) over the Schwartz function $h$ being part of the implied constant. Hence bounding (6.8), using (6.14) and (6.15), we finally conclude Lemma 6.2

\subsection{Large sieve inequalities for Bessel functions II}

For a complete proof of Theorem 6.1 we still owe justifications for Lemmata 6.3 and 6.4 . The purpose of this section is to provide detailed proofs for both results. We begin with the first of those. When estimating $\Delta_{X}^{-}(\alpha)$ in the form of 6.9 we encounter a term involving the $K$-Bessel function. We recall that from 7.31) we know that we may write this function as

$$
K_{\mu}(x)=\frac{1}{2 \pi \mathrm{i}} \int_{(\sigma)} 2^{\nu-2} \Gamma\left(\frac{\nu-\mu}{2}\right) \Gamma\left(\frac{\nu+\mu}{2}\right) x^{-\nu} d \nu,
$$

for every $\sigma>\Re \mu \geq 0$. In the context of the argument we then have to understand Lemma 6.3, which says that for $\nu \in \mathbb{C}$, odd prime level $N$ and $Z, R \geq 1$ the sum

$$
\mathcal{N}_{1}(Z, R ; \nu)=\sum_{\substack{Z<t \leq 4 Z \\(t, N)=1}} \sum_{R<h \leq 2 R}\left|\sum_{M<n \leq 2 M} a_{n} S( \pm h \bar{N}, n, t)\left(\frac{t^{2} N}{h n}\right)^{\nu / 2}\right|^{2}
$$

admits for every $\alpha \in \mathbb{C}_{M}^{\infty}$ the bound

$$
\|\alpha\|^{2}\left(\frac{Z^{2} N}{R M}\right)^{\Re \nu}\left(R Z^{2}+M R+Z^{3}+Z M\right) .
$$


In our setting there is a connection between the sum $\mathcal{N}_{1}(Z, R ; \nu)$ and the $K$-Bessel function. Writing the latter as its own inverse Mellin transform, as stated in 6.16), its argument $x^{-\nu}$ under the integral is reflected in the additional term in the square in (6.17), which we sum against a Kloosterman sum an the entries of the vector $\alpha$. Although the quality of the upper bound provided by Lemma 6.3 for the sum $\mathcal{N}_{1}(Z, R ; \nu)$ originates from sign changes of the Kloosterman sums we consider in the square in (6.17), rather than the involvement of the $K$-Bessel function, one might argue that this large sieve inequality is one for Kloosterman sums and not Bessel functions. However the $K$-Bessel function as given in $(6.16)$, which we recall is absolutely convergent for any $\sigma>\Re \mu \geq 0$, (see Lemma 7.9), offers some flexibility in the estimate in (6.18). The choice of the contour along which we integrate in (6.16) resonates in the exponent of the middle factor in 6.18). We have already exploited this previously in this chapter when employing Lemma 6.3. The significance of the $K$-Bessel function in this context is the reason, for establishing the desired estimate of 6.18 for $\mathcal{N}_{1}(Z, R ; \nu)$ in the course of this particular section. The general idea and strategy for the proof of Lemma 6.3 is based on the ansatz and strategy used in the argument establishing [15. Lemma 11.1, (for statement and proof see p. 558)]. Although in many aspects similar, our proof of Lemma 6.3 is designed to treat a slightly different situation than in the [15, proof of Lemma 11.1, p. 558] and thus we retrace the argument to give a full proof in support of our lemma with adjustments to our context.

Proof of Lemma 6.3. As discussed we want to exploit the sign changes of the Kloosterman sum in (6.17) in order to obtain a good bound for $\mathcal{N}_{1}(Z, R ; \nu)$ for our purposes. We will employ a large sieve for additive characters to help achieve this goal. Working in this direction, by positivity we enlarge the expression in 6.17 by letting the $t$-summation run over the full interval $(Z, 2 Z]$. Then opening the square shows that $\mathcal{N}_{1}(Z, R ; \nu)$ is at most

$$
\left(\frac{Z^{2} N}{R}\right)^{\Re \nu} \sum_{Z<t \leq 4 Z} \sum_{R<h \leq 2 R} \sum_{M<m, n \leq 2 M} b_{n} \bar{b}_{m} S( \pm h \bar{N}, n, t) S( \pm h \bar{N}, m, t)\left(\frac{m}{n}\right)^{\mathrm{i} \Im \nu / 2},
$$

where we define $b_{n}:=a_{n} n^{-\Re \nu / 2}$. Plugging in the definition for the Kloosterman sums we realise that the exponential, with the variable $h$ in its argument, is periodic with period $t$. Thus it suffices to consider one interval of length $t$, i.e. we will sum once over the residue classes $h(\bmod t)$, and then multiply by the number of such intervals, for $h$ running itself in an interval of length $R$. There are at most $[R / t]+1$ such intervals and so we estimate the latter bound further by

$$
\begin{aligned}
\left(\frac{Z^{2} N}{R}\right)^{\Re \nu} \sum_{Z<t \leq 4 Z}\left(\frac{R}{t}+1\right) . & \\
& \sum_{\substack{x_{1} \bmod t \\
x_{2} \bmod t}}^{*} \sum_{M<m, n \leq 2 M} b_{n} \bar{b}_{m} e\left(\frac{\bar{x}_{1} n-\bar{x}_{2} m}{t}\right)\left(\frac{m}{n}\right)^{\mathrm{i} \Im \nu / 2} \sum_{h \bmod t} e\left( \pm \frac{h \bar{N}\left(x_{1}-x_{2}\right)}{t}\right),
\end{aligned}
$$

where we used that the Kloosterman sums are real to be able to change the sign in one of the exponentials. We point out that $\left(\bar{N}\left(x_{1}-x_{2}\right), t\right)=1$, for non-equal residue classes $x_{1}, x_{2}$ of modulus $t$. Thus using the classic fact that

$$
\sum_{h \bmod t} e\left( \pm \frac{h \bar{N}\left(x_{1}-x_{2}\right)}{t}\right)= \begin{cases}t, & \text { if } x_{1}=x_{2} \\ 0, & \text { otherwise }\end{cases}
$$

we can reduce to the diagonal term of the summations of the residue classes and form again a square. We obtain

$$
\mathcal{N}_{1}(Z, R ; \nu) \ll\left(\frac{Z^{2} N}{R}\right)^{\Re \nu}(R+Z) \sum_{Z<t \leq 4 Z} \sum_{x \bmod t}^{*}\left|\sum_{M<n \leq 2 M} b_{n} e\left(\frac{x n}{t}\right) n^{-\mathrm{i} \Im \nu / 2}\right|^{2}
$$


Estimating the latter sum using the large sieve inequality for additive characters as given in [14, Theorem 7.11], we quickly arrive at the statement of the lemma.

Now we turn our attention to Lemma 6.4. We recall the statement of the result, which provides for $1 \leq$ $Z \leq M / N$ and $1 \leq R \leq 327(M / N)^{1+\delta}$, with odd prime level $N$, and for every $\alpha \in \mathbb{C}_{M}^{\infty}$ for the sum

$$
\mathcal{N}_{2}(Z, R ; \nu):=\sum_{\substack{Z<t \leq 2 Z \\(t, N)=1}} \sum_{R<h \leq 2 R}\left|\sum_{M<n \leq 2 M} a_{n} S( \pm h \bar{N}, n, t) B_{\nu}\left(\frac{4 \pi}{t} \sqrt{\frac{h n}{N}}\right)\right|^{2}
$$

the upper bound

$$
\|\alpha\|^{2} N Z^{2}\left(1+\frac{M}{N^{2}}+\left(\frac{M}{R N}\right)^{1 / 2}\right) M^{\varepsilon} e^{3 \pi|\Im \nu|} .
$$

We highlight the important fact that the function $B_{\nu}(x)$ may both denote the $Y$ or the $J$-Bessel function, each with index $\nu \in \mathrm{i} \mathbb{R}$ and argument $x \in \mathbb{R}_{>0}$. Thus the statement of the lemma encompasses two large sieve inequalities. Our proof of Lemma 6.4 follows closely the ansatz, overall strategy, oultine as well as individual steps of the argument presented in [15, 12. Large sieve for absolute Kloosterman sums (dual approach), p.558-561] proving [15, Proposition 12.1, p. 561]. However Lemma 6.4describes a somewhat different scenario compared to the one of the paper just quoted and thus we will retrace the steps of the proof in detail, adapting it to our situation.

Proof of Lemma 6.4 There are several ways to approach an estimation of the sum $\mathcal{N}_{2}(Z, R ; \nu)$. By the Duality Principle (cp. [14, p. 170]) we know that a bound of its dual sum

$$
\widetilde{\mathcal{N}}_{2}(Z, R ; \nu) \ll \Omega\|\beta\|^{2},
$$

for any complex vector $\beta$ and a quantity $\Omega>0$, immediately implies the bound

$$
\mathcal{N}_{2}(Z, R ; \nu) \ll \Omega\|\alpha\|^{2},
$$

for any complex vector $\alpha$ and with the same $\Omega$. Thus, with this relationship between bounds for the original sum and its dual sum in mind, instead of working with the $\operatorname{sum} \mathcal{N}_{2}(Z, R ; \nu)$ as given in the statement, we can make progress towards an estimate by choosing to consider its dual sum

$$
\widetilde{\mathcal{N}}_{2}(Z, R ; \nu):=\sum_{M<n \leq 2 M}\left|\sum_{\substack{Z<t \leq 2 Z \\(t, N)=1}} \sum_{R<h \leq 2 R} b(t, h) S( \pm h \bar{N}, n, t) B_{\nu}\left(\frac{4 \pi}{t} \sqrt{\frac{h n}{N}}\right)\right|^{2}
$$

instead. In accordance with the statement of the Duality Principle we have to be able to control this sum for any complex vector

$$
\beta \in \mathbb{C}_{Z_{N}^{*}, R}^{\infty \times \infty}:=\left\{\beta=(b(t, h))_{t, h \in \mathbb{N}} \in \mathbb{C}^{\infty \times \infty} \mid \operatorname{supp}(\beta) \subseteq Z_{N}^{*} \times(R, 2 R]\right\},
$$

where we define the set $Z_{N}^{*}:=\{t \in(Z, 2 Z] \mid(t, N)=1\}$. To do exactly that we will follow the strategy where we, after multiplying out the square in the dual sum, replace the $n$-summation essentially with an integral. Exploiting oscillation of the functions in the integrand, by an integration by parts argument we may find estimates for the whole dual sum of the desired magnitude. Such an argument ideally requires the integrand, of the integral we work with, to have compact support. In order to find ourselves in such a scenario after transforming the $n$-summation in a corresponding integral, we therefore want a smooth cut 
off of the summation boundaries therein. To this end we introduce the smooth cut-off function $v: \mathbb{R} \rightarrow \mathbb{R}_{\geq 0}$ with $\operatorname{supp}(v) \subseteq[3 / 4,9 / 4]$, which equals one on $[1,2]$. Beginning to execute the general strategy just laid out, we now estimate the dual sum by

$$
\sum_{n=-\infty}^{\infty} v\left(\frac{n}{M}\right)\left|\sum_{\substack{Z<t \leq 2 Z \\(t, N)=1}} \sum_{R<h \leq 2 R} \cdots\right|^{2}
$$

which, after opening the square, is

$$
\sum_{t_{1}, h_{1}} \sum_{t_{2}, h_{2}} \sum_{\substack{x_{1} \text { mod } t_{1} \\ x_{2} \text { mod } t_{2}}}^{*} b\left(t_{1}, h_{1}\right) \bar{b}\left(t_{2}, h_{2}\right) e\left( \pm \bar{N}\left(\frac{h_{1} \bar{x}_{1}}{t_{1}}-\frac{h_{2} \bar{x}_{2}}{t_{2}}\right)\right) \mathfrak{B}_{h_{1}, h_{2}}\left(t_{1}, t_{2}, x_{1}, x_{2}\right) .
$$

Here we abbreviate

$$
\mathfrak{B}_{h_{1}, h_{2}}\left(t_{1}, t_{2}, x_{1}, x_{2}\right):=\sum_{n=-\infty}^{\infty} v\left(\frac{n}{M}\right) e\left(n\left\|\frac{x_{1}}{t_{1}}-\frac{x_{2}}{t_{2}}\right\|\right) B_{\nu}\left(\frac{4 \pi}{t_{1}} \sqrt{\frac{h_{1} n}{N}}\right) \bar{B}_{\nu}\left(\frac{4 \pi}{t_{2}} \sqrt{\frac{h_{2} n}{N}}\right),
$$

where it is enough to consider the distance to the nearest integer of the difference of the fractions in the argument of the exponential. This can be seen, noticing that since the latter is periodic with period one, it suffices to consider only the fractional part of the difference of the fractions. By changing the order of summation of the two sums over the residue classes, respectively of moduli $t_{1}$ and $t_{2}$, indeed we see that it suffices to consider the distance to the nearest integer. Now our goal becomes to replace the sum 6.21 by an integral at the cost of a small error. The statement we want to justify reads in precise terms as follows.

Lemma 6.5. For $M \geq 96 R /\left(Z^{2} N\right)$ we have the asymptotic expansion

$$
\mathfrak{B}_{h_{1}, h_{2}}\left(t_{1}, t_{2}, x_{1}, x_{2}\right)=\mathfrak{I}_{h_{1}, h_{2}}\left(t_{1}, t_{2}, x_{1}, x_{2}\right)+\mathcal{O}\left(\frac{e^{3 \pi|\Im \nu|}}{M^{j}}\right),
$$

for every $j \geq 1$, where

$$
\mathfrak{I}_{h_{1}, h_{2}}\left(t_{1}, t_{2}, x_{1}, x_{2}\right):=\int_{\mathbb{R}} v\left(\frac{y}{M}\right) e\left(y\left\|\frac{x_{1}}{t_{1}}-\frac{x_{2}}{t_{2}}\right\|\right) B_{\nu}\left(\frac{4 \pi}{t_{1}} \sqrt{\frac{h_{1} y}{N}}\right) \bar{B}_{\nu}\left(\frac{4 \pi}{t_{2}} \sqrt{\frac{h_{2} y}{N}}\right) d y .
$$

Before we justify the lemma we highlight the fact that the implied constant in the asymptotic, given in the latter lemma, does not depend on the index of the Bessel functions $\nu$. This is important since it is a necessary feature for us to proceed with our goal to obtain bounds for the dual sum $\widetilde{\mathcal{N}}_{2}(Z, R ; \nu)$, which are uniform in the index. The following argument supports the lemma. First we observe that by Poisson summation the sum (6.21) is equal to

$$
\sum_{k=-\infty}^{\infty} \int_{\mathbb{R}} v\left(\frac{y}{M}\right) e\left(y\left\|\frac{x_{1}}{t_{1}}-\frac{x_{2}}{t_{2}}\right\|\right) B_{\nu}\left(\frac{4 \pi}{t_{1}} \sqrt{\frac{h_{1} y}{N}}\right) \bar{B}_{\nu}\left(\frac{4 \pi}{t_{2}} \sqrt{\frac{h_{2} y}{N}}\right) e(-k y) d y .
$$

For $k=0$ we obtain the desired integral $\mathfrak{I}_{h_{1}, h_{2}}\left(t_{1}, t_{2}, x_{1}, x_{2}\right)$, which is the main term in the asymptotic claimed in the lemma. It remains to show that the rest of the summation

$$
\sum_{k \in \mathbb{Z} \backslash\{0\}} \mathrm{I}(k),
$$


where $\mathrm{I}(k)$ denotes the integral in 6.22 , is indeed small. This will be accomplished by exploiting the occurring oscillation via integrating by parts. We use Lemma 7.7 to dismember the Bessel functions involved into oscillating parts and such, which are smooth and admit, as well as their derivatives, suitable bounds. Precisely we find that $\mathrm{I}(k)$ is

$$
\begin{aligned}
& \frac{1}{8 \pi^{2}}\left(\frac{t_{1} t_{2} N}{\sqrt{h_{1} h_{2}}}\right)^{1 / 2}\left\{\hat{x} \sum_{ \pm} \int_{\mathbb{R}} v\left(\frac{y}{M}\right) e\left( \pm 2 \sqrt{\frac{y}{N}}\left(\frac{\sqrt{h_{1}}}{t_{1}}+\frac{\sqrt{h_{2}}}{t_{2}}\right)+y\left(\left\|\frac{x_{1}}{t_{1}}-\frac{x_{2}}{t_{2}}\right\|-k\right)\right) .\right. \\
& \cdot \Omega_{ \pm}\left(\nu, \frac{2}{t_{1}} \sqrt{\frac{h_{1} y}{N}}\right) \bar{\Omega}_{\mp}\left(\nu, \frac{2}{t_{2}} \sqrt{\frac{h_{2} y}{N}}\right) \frac{d y}{\sqrt{y}} \\
& +\sum_{ \pm} \int_{\mathbb{R}} v\left(\frac{y}{M}\right) e\left( \pm 2 \sqrt{\frac{y}{N}}\left(\frac{\sqrt{h_{1}}}{t_{1}}-\frac{\sqrt{h_{2}}}{t_{2}}\right)+y\left(\left\|\frac{x_{1}}{t_{1}}-\frac{x_{2}}{t_{2}}\right\|-k\right)\right) . \\
& \left.\cdot \Omega_{ \pm}\left(\nu, \frac{2}{t_{1}} \sqrt{\frac{h_{1} y}{N}}\right) \bar{\Omega}_{ \pm}\left(\nu, \frac{2}{t_{2}} \sqrt{\frac{h_{2} y}{N}}\right) \frac{d y}{\sqrt{y}}\right\}
\end{aligned}
$$

where the symbol 1 denotes a plus sign in the case of $B_{\nu}$ being the $J$-Bessel function and a minus sign if $B_{\nu}$ is the $Y$-Bessel function. Since we know that $M \geq 96 R /\left(Z^{2} N\right)$ is large enough in comparison to the other variables involved, we observe that the first derivative of the phase function of the exponentials

$$
\mathrm{H}_{ \pm, \pm}(y ; k):= \pm 4 \pi \sqrt{\frac{y}{N}}\left(\frac{\sqrt{h_{1}}}{t_{1}} \pm \frac{\sqrt{h_{2}}}{t_{2}}\right)+2 \pi y\left(\left\|\frac{x_{1}}{t_{1}}-\frac{x_{2}}{t_{2}}\right\|-k\right)
$$

never equals zero, so there is no stationary phase. Notice that the definition of $\mathrm{H}_{ \pm, \pm}(y ; k)$ represents all four possible combinations of plus and minus signs. We see immediately

$$
\left|\frac{\partial}{\partial y} \mathrm{H}_{ \pm, \pm}(y ; k)\right| \geq|k|-5 / 6
$$

which of course is always positive, since $|k| \geq 1$. Furthermore for $y \in \operatorname{supp}(v)$ we also find the bounds

$$
\frac{\partial^{j}}{\partial y^{j}} \mathrm{H}_{ \pm, \pm}(y ; k) \ll \frac{1}{M^{j-1}}, \text { for } j \geq 2 .
$$

Finally we define the function

$$
\Upsilon_{ \pm, \pm}(y):=v\left(\frac{y}{M}\right) \Omega_{ \pm}\left(\nu, \frac{2}{t_{1}} \sqrt{\frac{h_{1} y}{N}}\right) \bar{\Omega}_{ \pm}\left(\nu, \frac{2}{t_{2}} \sqrt{\frac{h_{2} y}{N}}\right) y^{-1 / 2},
$$

where this function represents again all four possible combination of plus and minus signs. Employing Lemma 7.7 to estimate the functions $\Omega_{ \pm}$and their conjugates, we obtain for $j \geq 0$ that

$$
\Upsilon_{ \pm, \pm}^{(j)}(y) \ll \frac{e^{3 \pi|\Im \nu|}}{M^{j+1 / 2}}
$$

also for $y \in \operatorname{supp}(v)$. Now [1, Lemma 8.1] provides the same upper bound for each integral in 6.23) and thus this also gives us an estimate for $\mathrm{I}(k)$ upon invoking that $Z \leq M / N$ and $R, N \geq 1$. Form there we conclude the bound for the error term

$$
\sum_{k \in \mathbb{Z} \backslash\{0\}} \mathrm{I}(k) \ll \frac{e^{3 \pi|\Im \nu|}}{M^{j}}\left(1+\sum_{|k| \geq 2} \frac{1}{(|k|-5 / 6)^{4}}\right) \ll \frac{e^{3 \pi|\Im \nu|}}{M^{j}}
$$


for every $j \geq 1$. This finishes the argument establishing Lemma 6.5 Now we can continue with our original estimation of (6.19). To this end we will distinguish the two scenarios when

$$
\left\|\frac{x_{1}}{t_{1}}-\frac{x_{2}}{t_{2}}\right\|>0 \text { and when }\left\|\frac{x_{1}}{t_{1}}-\frac{x_{2}}{t_{2}}\right\|=0 .
$$

The latter case implies that

$$
\frac{x_{1}}{t_{1}} \equiv \frac{x_{2}}{t_{2}}(\bmod 1)
$$

from which we conclude that in fact the only instance this can occur for invertible residue classes $x_{1}$ and $x_{2}$ of moduli $t_{1}$ and $t_{2}$ respectively, is precisely for $x_{1}=x_{2}$ and $t_{1}=t_{2}$. We will begin by treating these diagonal terms first and deal with the off-diagonal terms of 6.20 later. The diagonal term is given by

$$
\tilde{\mathcal{N}}_{2}^{\text {diag }}(Z, R ; \nu):=\sum_{n=-\infty}^{\infty} v\left(\frac{n}{M}\right) \sum_{\substack{Z<t \leq 2 Z \\(t, N)=1}} \sum_{x \bmod t}^{*}\left|\sum_{R<h \leq 2 R} b(t, h) e\left( \pm \frac{\bar{N} x h}{t}\right) B_{\nu}\left(\frac{4 \pi}{t} \sqrt{\frac{h n}{N}}\right)\right|^{2} .
$$

By positivity we may find an upper bound for the diagonal term by enlarging the latter sum by letting it run over all residue classes, not only the invertible ones. Then we want to replace the $n$-summation by an integral. Thus Lemma 6.5 comes into play and after opening the square we replace the sum $\mathfrak{B}_{h_{1}, h_{2}}(t, t, x, x)$ by the integral stated in the lemma. Since $(N, t)=1$ we observe that

$$
\sum_{x \bmod t} e\left( \pm \frac{x \bar{N}\left(h_{1}-h_{2}\right)}{t}\right)= \begin{cases}t, & \text { if } h_{1} \equiv h_{2}(\bmod t) \\ 0, & \text { otherwise }\end{cases}
$$

and thus obtain for any $j \geq 1$ that

$$
\widetilde{\mathcal{N}}_{2}^{\text {diag }}(Z, R ; \nu) \ll \sum_{Z<t \leq 2 Z} t \sum_{R<h_{1} \leq 2 R} \sum_{\substack{R<h_{2} \leq 2 R \\ h_{2}=h_{1} \bmod t}}\left|b\left(t, h_{1}\right) \bar{b}\left(t, h_{2}\right) \Im_{h_{1}, h_{2}}(t)\right|+\|\beta\|^{2} e^{3 \pi|\Im \nu|} M^{-j},
$$

where for brevity we write $\mathfrak{I}_{h_{1}, h_{2}}(t, t, x, x):=\mathfrak{I}_{h_{1}, h_{2}}(t)$, since the integral does not depend on the residue classes of modulus $t$ anymore and where we estimate the vector $\beta \in \mathbb{C}_{Z_{N}^{*}, R}^{\infty \times \infty}$ with the norm

$$
\|\beta\|:=\left(\sum_{t \in Z_{N}^{*}} \sum_{R<h \leq 2 R}|b(t, h)|^{2}\right)^{1 / 2} .
$$

For this integral we now have to establish a useful bound for our purposes. We will do this in several steps. First of all for any small $\varepsilon>0$ we estimate

$$
B_{\nu}\left(\frac{4 \pi}{t} \sqrt{\frac{h_{1} y}{N}}\right) \bar{B}_{\nu}\left(\frac{4 \pi}{t} \sqrt{\frac{h_{2} y}{N}}\right) \ll e^{2 \pi|\Im \nu|}\left(1+|\Im \nu|^{3}\right) M^{\varepsilon} \min \left\{1,\left(\frac{Z^{2} N}{R M}\right)^{1 / 2}\right\},
$$

for purely imaginary index $\nu \in \mathrm{i} \mathbb{R}$ and $R, N \geq 1$ and $Z \leq M / N$. In order to obtain the latter we used Corollary 7.3 to treat the case of $B_{\nu}$ being the $J$-Bessel function and Lemma 7.4 in case it is the $Y$-Bessel function. As a direct consequence we now find the trivial estimate

$$
\Im_{h_{1}, h_{2}}(t) \ll e^{3 \pi|\Im \nu|} M^{1+\varepsilon} \min \left\{1,\left(\frac{Z^{2} N}{R M}\right)^{1 / 2}\right\},
$$


for any small $\varepsilon>0$. Now, if we encounter that $h_{1} \neq h_{2}$ we may integrate $\mathfrak{I}_{h_{1}, h_{2}}(t)$ by parts. To this end we use Lemma 7.7 to decompose the Bessel functions into oscillating and only smooth parts, that possess easily controllable derivatives. Thus we see that, similarly to 6.23 , it suffices to bound

$$
\left(\frac{t^{2} N}{\sqrt{h_{1} h_{2}}}\right)^{1 / 2} \int_{\mathbb{R}} v\left(\frac{y}{M}\right) e\left( \pm 2 \frac{\sqrt{y}\left(\sqrt{h_{1}}-\sqrt{h_{2}}\right)}{t \sqrt{N}}\right) \Omega_{ \pm}\left(\nu, \frac{2}{t} \sqrt{\frac{h_{1} y}{N}}\right) \bar{\Omega}_{ \pm}\left(\nu, \frac{2}{t} \sqrt{\frac{h_{2} y}{N}}\right) \frac{d y}{\sqrt{y}},
$$

in order to determine an estimate for $\mathfrak{I}_{h_{1}, h_{2}}(t)$. As before we quickly find that for the phase function of the exponential

$$
\mathrm{H}_{ \pm}(y):= \pm 4 \pi \frac{\sqrt{y}\left(\sqrt{h_{1}}-\sqrt{h_{2}}\right)}{t \sqrt{N}}
$$

we have the bound

$$
\left|\mathrm{H}_{ \pm}^{\prime}(y)\right| \gg \frac{\left|\sqrt{h_{1}}-\sqrt{h_{2}}\right|}{Z \sqrt{N M}},
$$

which is never zero, unless $h_{1}=h_{2}$, which we have excluded in this situation. Furthermore we also have the upper bounds

$$
\mathrm{H}_{ \pm}^{(j)}(y) \ll \frac{\left|\sqrt{h_{1}}-\sqrt{h_{2}}\right|}{Z} \sqrt{\frac{M}{N}} \cdot \frac{1}{M^{j}}, \quad \text { for } \quad j \geq 0 .
$$

For the remaining integrand of $\mathfrak{I}_{h_{1}, h_{2}}(t)$ we define

$$
\Upsilon_{ \pm}(y):=v\left(\frac{y}{M}\right) \Omega_{ \pm}\left(\nu, \frac{2}{t} \sqrt{\frac{h_{1} y}{N}}\right) \bar{\Omega}_{ \pm}\left(\nu, \frac{2}{t} \sqrt{\frac{h_{2} y}{N}}\right) y^{-1 / 2},
$$

for which we recall the estimates

$$
\Upsilon_{ \pm}^{(j)}(y) \ll \frac{e^{3 \pi|\Im \nu|}}{M^{j+1 / 2}}, \text { for } j \geq 0 .
$$

Employing the above bounds for the derivatives of the phase function $\mathrm{H}_{ \pm}$and for the function $\Upsilon_{ \pm}$, integrating $(6.29)$ by parts twice we establish that

$$
\Im_{h_{1}, h_{2}}(t) \ll e^{3 \pi|\Im \nu|}\left(\frac{Z^{2} N}{R M}\right)^{1 / 2} M\left(\frac{\left|\sqrt{h_{1}}-\sqrt{h_{2}}\right|}{Z} \sqrt{\frac{M}{N}}\right)^{-2} .
$$

We observe that for $Z^{2} N /(R M) \geq 1$ we have

$$
\frac{\left|\sqrt{h_{1}}-\sqrt{h_{2}}\right|}{Z} \sqrt{\frac{M}{N}}<1
$$

so the estimate 6.28 is more favourable in comparison to 6.30 and thus merging the two bounds we conclude

$$
\mathfrak{I}_{h_{1}, h_{2}}(t) \ll \frac{e^{3 \pi|\Im \nu|} M^{1+\varepsilon} \cdot \min \left\{1,\left(\frac{Z^{2} N}{R M}\right)^{1 / 2}\right\}}{1+\left(\frac{h_{1}-h_{2}}{Z} \sqrt{\frac{M}{R N}}\right)^{2}},
$$


where we used that $h_{i} \asymp R$ to modify the denominator. Equipped with the latter estimate we may now control the integral in 6.27). This tells us that the diagonal term $\widetilde{\mathcal{N}}_{2}^{\text {diag }}(Z, R ; \nu)$ does not exceed

$$
\frac{e^{3 \pi|\Im \nu|} M^{\varepsilon} Z^{2}\left(\frac{N M}{R}\right)^{1 / 2}}{\left(1+\frac{Z^{2} N}{R M}\right)^{1 / 2}} \sum_{Z<t \leq 2 Z} \sum_{R<h_{1} \leq 2 R} \sum_{\substack{R<h_{2} \leq 2 R \\ h_{2} \equiv h_{1} \bmod t}} \frac{\left|b\left(t, h_{1}\right) \bar{b}\left(t, h_{2}\right)\right|}{1+\left(\frac{h_{1}-h_{2}}{Z} \sqrt{\frac{M}{R N}}\right)^{2}}+\frac{\|\beta\|^{2} e^{3 \pi|\Im \nu|}}{M} .
$$

It remains to bound the summations in the estimate just established. Since we only consider such terms in the $h_{2}$ summation for which $h_{2} \equiv h_{1}(\bmod t)$, we may write $h_{2}=h_{1}+f t$, for $f \in \mathbb{N}_{0}$, and sum over $f$ instead. Since $t \asymp Z$ and after an application of the Cauchy Schwarz inequality we quickly see that the terms that are summed in 6.31) are bounded by

$$
\|\beta\| \cdot \sum_{f=0}^{\infty} \frac{1}{1+\left(f \sqrt{\frac{M}{R N}}\right)^{2}}\left(\sum_{Z<t \leq 2 Z} \sum_{R<h_{1} \leq 2 R}\left|\bar{b}\left(t, h_{1}+t f\right)\right|\right)^{1 / 2} .
$$

Since we know for the vector that $\operatorname{supp}(\beta) \subseteq Z_{N}^{*} \times(R, 2 R]$, we in turn observe that the latter is dominated via a change of variables by

$$
\|\beta\|^{2}\left(1+\int_{0}^{\infty} \frac{d x}{1+\left(x \sqrt{\frac{M}{R N}}\right)^{2}}\right) \ll\|\beta\|^{2}\left(1+\sqrt{\frac{R N}{M}}\right) .
$$

With the latter estimate applied in 6.31 we arrive immediately at

$$
\widetilde{\mathcal{N}}_{2}^{\text {diag }}(Z, R ; \nu) \ll\|\beta\|^{2} M^{\varepsilon} Z^{2} N\left(\frac{1+\frac{M}{R N}}{1+\frac{Z^{2} N}{R M}}\right)^{1 / 2} e^{3 \pi|\Im \nu|} .
$$

This finishes our considerations regarding the diagonal term. We are left with estimating the off-diagonal terms in (6.20). Form (6.26) we know that for such terms the distance to the nearest integer of the difference of the two fractions involved is positive. Hence, recalling that $Z<t \leq 2 Z$, we have the lower bound

$$
\left\|\frac{x_{1}}{t_{1}}-\frac{x_{2}}{t_{2}}\right\| \geq \frac{1}{4 Z^{2}}
$$

Now we use Lemma 6.5 to replace the $n$-summation in 6.20 with the integral $\mathfrak{I}_{h_{1}, h_{2}}\left(t_{1}, t_{2}, x_{1}, x_{2}\right)$. The off-diagonal terms $\widehat{\mathcal{N}_{2}^{\text {offdiag }}}(Z, R ; \nu)$ are therefore dominated by

$$
\sum_{t_{1}, h_{1}} \sum_{t_{2}, h_{2}} \sum_{\substack{x_{1} \text { mod } t_{1} \\ x_{2} \text { mod } t_{2}}}^{*}\left|b\left(t_{1}, h_{1}\right) \bar{b}\left(t_{2}, h_{2}\right) \Im_{h_{1}, h_{2}}\left(t_{1}, t_{2}, x_{1}, x_{2}\right)\right|+\|\beta\|^{2} e^{3 \pi|\Im \nu|} M^{-j},
$$

for any choice of $j \geq 1$. Just as before in the case of the diagonal terms we proceed by estimating the integral in the bound just established. By a repeated integration by parts argument we will see that this is in fact small. Before we integrate we want to be able to take the oscillation of the Bessel functions into account. Thus as before with Lemma 7.7 we can rewrite those, obtaining a result that is as in [6.23), but with $k=0$. We encounter the phase function $\mathrm{H}_{ \pm, \pm}(y ; 0)$ of the exponentials and the function $\Upsilon_{ \pm, \pm}(y)$ also present under the integral sign, both functions as defined in 6.24$)$ and 6.25$)$ respectively. Again we need appropriate bounds for both functions in order to be able to integrate by parts. We want to do this without 
having to deal with stationary phase in the integral. Thus for a quantity $W \geq 1$, which we will determine more precisely at a later stage, we restrict to the case of

$$
M \geq Z^{2}\left(\frac{216 R}{N}+W\right) .
$$

We remark that the inequality in 6.35 automatically always implies the lower bound for $M$, which we demanded in the conditions of Lemma 6.5. Now, since with 6.35) we know that $M$ is not too small, together with the lower bound in 6.33 , we find that

$$
\left|\mathrm{H}_{ \pm, \pm}^{\prime}(y ; 0)\right| \geq \frac{\pi}{25} \cdot \frac{1}{Z^{2}},
$$

which is always positive and so indeed there is no stationary phase in the integral. Furthermore we have, similarly as before in the argument supporting Lemma 6.5 , the bounds

$$
\mathrm{H}_{ \pm, \pm}^{(j)}(y ; 0) \ll \frac{M}{Z^{2}} \cdot \frac{1}{M^{j}}, \text { for } j \geq 2, \text { and } \Upsilon_{ \pm, \pm}^{(j)}(y) \ll \frac{e^{3 \pi|\Im \nu|}}{M^{j+1 / 2}}, \text { for } j \geq 0,
$$

where the estimate for the derivatives of $\mathrm{H}_{ \pm, \pm}(y ; 0)$ again relies on 6.35 . The just derived bounds enable us to make use of [1, Lemma 8.1], from which we now find that

$$
\Im_{h_{1}, h_{2}}\left(t_{1}, t_{2}, x_{1}, x_{2}\right) \ll e^{3 \pi|\Im \nu|} M\left(\frac{Z^{2} N}{R M}\right)^{1 / 2}\left(\frac{M}{Z^{2}}\right)^{-j} \ll \frac{e^{3 \pi|\Im \nu|} M^{3 / 2}}{W^{j}},
$$

for any $j \geq 1$ and under the assumption of 6.35). Applying the latter upper bound for the integral $\mathfrak{I}_{h_{1}, h_{2}}\left(t_{1}, t_{2}, x_{1}, x_{2}\right)$ in 6.34 and after an application of the Cauchy Schwarz inequality, we immediately arrive at

$$
\tilde{\mathcal{N}}_{2}^{\text {offdiag }}(Z, R ; \nu) \ll\|\beta\|^{2} \frac{M^{3 / 2} Z^{3} R}{W^{j}} e^{3 \pi|\Im \nu|},
$$

for any $j \geq 1$ and where we summarise easily with the error arising form the application of Lemma 6.5. upon using again 6.35. This finishes the bound for the dual sum, since from the latter estimate in combination with the bound for the diagonal contribution as given in 6.32 we finally see that

$$
\widetilde{\mathcal{N}}_{2}(Z, R ; \nu) \ll\|\beta\|^{2}\left(\frac{M^{3 / 2} Z^{3} R}{W^{j}}+M^{\varepsilon} Z^{2} N\left(\frac{1+\frac{M}{R N}}{1+\frac{Z^{2} N}{R M}}\right)^{1 / 2}\right) e^{3 \pi|\Im \nu|},
$$

for any choice of $\beta \in \mathbb{C}_{Z_{N}^{*}, R}^{\infty \times \infty}$. From here we immediately obtain the same bound for the sum $\mathcal{N}_{2}(Z, R ; \nu)$, of course proportional to the 2-norm squared of the vector $\alpha$ instead of $\beta$, which we originally set out to estimate. As already mentioned at the beginning of the proof, this is a consequence of the duality principle. The obtained bound however, is so far only valid under the obstruction that

$$
M \geq Z^{2}\left(\frac{216 R}{N}+W\right) .
$$

Our final goal is now to remove this restriction. This will follow in exactly the same fashion as in [15, p. 560/561, 12. Large sieve for absolute Kloosterman sums (dual approach)] and in light of only an insignificant difference between our scenario and the one in the paper just quoted, we omit further details here[(1) As in the paper [15] we choose $W=M^{\varepsilon}$, for any small $\varepsilon>0$, and finally conclude the statement of Lemma 6.4 which finishes this section.

\footnotetext{
${ }^{\text {(I) }}$ Because our condition 6.35, which we aim to remove, differs in a constant, also the constants involved when choosing $P$, as in the paper [15], have to be different. We require $P$ to be a little bit larger than before, for instance $P=W Z^{2} / M+18 Z(R /(N M))^{1 / 2}+$ $\log Z$ works in our situation.
} 


\section{Chapter 7}

\section{Appendix}

In quite a few instances throughout this thesis, progress in the calculations depends on understanding the behaviour of the $J, Y$ and $K$-Bessel functions. Because of their significance we are going to give some useful results about them for our purposes.

\subsection{The $J$ and $Y$-Bessel function}

\subsubsection{Bounds for the $J$ and $Y$-Bessel function}

Lemma 7.1. For $x \in \mathbb{R}_{>0}$ let $J_{\nu}(x)$ be the J-Bessel function for $\nu \in \mathbb{C}$ with $\Re \nu \geq-1 / 2+\varepsilon$, for any small $\varepsilon>0$. Then we have

$$
J_{\nu}(x) \ll e^{\pi|\Im \nu| / 2}\left(\frac{x}{1+|\Im \nu|}\right)^{\Re \nu}
$$

Proof. From [10, 8.411.5] for $\Re \nu>-1 / 2$ we have

$$
J_{\nu}(x)=\frac{(x / 2)^{\nu}}{\Gamma(\nu+1 / 2) \Gamma(1 / 2)} \int_{0}^{\pi} \cos (x \cos \phi) \sin ^{2 \nu}(\phi) d \phi .
$$

Stirling approximation yields

$$
\Gamma^{-1}(\nu+1 / 2) \ll e^{\pi|\Im \nu| / 2}(1+|\Im \nu|)^{-\Re \nu} .
$$

Furthermore, for $\Re \nu \geq 0$ we immediately have the bound $\sin ^{2 \nu}(\phi) \ll 1$, for all $\phi \in[0, \pi]$, and the integral in 7.1 is bounded uniformly in $x$ by a constant. On the other hand in the case of $-1 / 2+\varepsilon \leq \Re \nu<0$ we have that the integral in 7.1 is bounded uniformly in $x$ by

$$
\int_{0}^{\pi}\left(\frac{1}{\sin \phi}\right)^{1-2 \varepsilon} d \phi \ll 1
$$

Once again the integral in 7.1 is bounded uniformly in $x$ by a constant. Combining the estimates for the gamma function and for the integral in (7.1) leads directly to the desired bound for the Bessel function.

Lemma 7.2. Let $J_{\nu}(k / x)$ be the J-Bessel function for a positive constant $k>0$ and assume that $\nu \in \mathbb{C}$ with $0 \leq \Re \nu<1$ and for $x \in \mathbb{R}_{>0}$. Then for any $j>0$ we have

$$
\frac{\partial^{j}}{\partial x^{j}} J_{\nu}\left(\frac{k}{x}\right) \ll e^{\pi|\Im \nu| / 2}\left(\frac{k}{x}\right)^{\Re \nu}\left\{1+\left(\frac{k}{x}\right)^{j+1}+\left(1+\frac{k}{x}\right)\left(|\nu|^{j}+x^{-j}+\left(\frac{|\nu|}{x}\right)^{j}+\left(\frac{k}{x^{2}}\right)^{j}\right)\right\},
$$


where the bound is uniform in $k$. In particular the bound becomes

$$
\frac{\partial^{j}}{\partial x^{j}} J_{\nu}\left(\frac{k}{x}\right) \ll e^{\pi|\Im \nu| / 2}\left(\frac{k}{x}\right)^{\Re \nu}\left\{1+|\nu|^{j}+x^{-j}+\left(\frac{|\nu|}{x}\right)^{j}\right\},
$$

provided that $k \leq x$.

Proof. We assume that $\Re \nu \geq 0$. From [11, Appendix: Bounds for Bessel functions, (100), p.649] we know that differentiating the Bessel function as above $j$-times yields

$$
\frac{\partial^{j}}{\partial x^{j}} J_{\nu}\left(\frac{k}{x}\right)=x^{-j} \sum_{l=0}^{j} P_{l}(x)\left(\frac{k}{x}\right)^{j-l} J_{\nu-j+l}\left(\frac{k}{x}\right) .
$$

This formula for the derivative features polynomials in $x$ of degree $l$, denoted by $P_{l}(x)$, with coefficients that depend on the total number of differentiations we perform and the degree of the polynomial. In order to bound this sum we need to know the size of each $J_{\nu-j+l}(k / x)$. However for Lemma 7.1 to become applicable in this context, first we need to bound the present Bessel functions in terms of such with index having a large enough real part. Using the recursion formula [10, 8.471.1], by induction on $m$ one establishes

$$
J_{\nu-m}\left(\frac{k}{x}\right) \ll\left(\left(\frac{|\nu-m|+1}{k / x}\right)^{m}+1\right)\left(\left|J_{\nu}\left(\frac{k}{x}\right)\right|+\left|J_{\nu+1}\left(\frac{k}{x}\right)\right|\right) \text {, for every } m \in \mathbb{N}_{0} .
$$

The condition $\Re \nu<1$ ensures that $|\nu-m| \geq|\nu-m+1|,|\nu-m+2|$, for every $m \geq 2$ and the base cases $m=0,1$ follow directly from the recursion formula just quoted. The latter two Bessel functions for an index with $\Re \nu \geq 0$ may therefore now be controlled using Lemma 7.1. Then applying this bound in (7.3) results in

$$
\begin{aligned}
\frac{\partial^{j}}{\partial x^{j}} J_{\nu}\left(\frac{k}{x}\right) & \ll e^{\pi|\Im \nu| / 2}\left(\frac{k}{x}\right)^{\Re \nu}\left(1+\frac{k}{x}\right) \sum_{l=0}^{j} \frac{\left|P_{l}(x)\right|}{x^{j}}\left((|\nu-(j-l)|+1)^{j-l}+\left(\frac{k}{x}\right)^{j-l}\right) \\
& \ll e^{\pi|\Im \nu| / 2}\left(\frac{k}{x}\right)^{\Re \nu}\left(1+\frac{k}{x}\right)\left(1+x^{-j}\right)\left(|\nu|^{j}+\left(\frac{k}{x}\right)^{j}+1\right),
\end{aligned}
$$

where we observed that

$$
\sum_{l=0}^{j}(|\nu-(j-l)|+1)^{j-l} \ll 1+|\nu|^{j} .
$$

Rearranging the bound in 7.4 gives us the lemma.

Corollary 7.3. Let $J_{\nu}(x)$ be the $J$-Bessel function with argument $x \in \mathbb{R}_{>0}$ and index $\nu \in \mathbb{C}$ with $|\Re \nu| \leq$ $1 / 2-\varepsilon$, for any small $\varepsilon>0$. Then we have

$$
J_{\nu}(x) \ll e^{\pi|\Im \nu|}\left(1+|\Im \nu|^{1 / 2}\right) \cdot \min \left\{x^{\Re \nu}, \frac{1}{\sqrt{x}}\right\} .
$$

Proof. The corollary is essentially just a summary of results established in this appendix. Form Lemma 7.7 we immediately conclude for $x>0$ and $|\Re \nu|<1 / 2$ the bound

$$
J_{\nu}(x) \ll \frac{e^{\pi|\Im \nu|}\left(1+|\Im \nu|^{1 / 2}\right)}{\sqrt{x}} .
$$

The decay in $x$ here is essentially optimal for large values of the argument. However for small $0<x \leq 1$ one can do better. In this situation Lemma 7.1 provides a better bound, for $-1 / 2+\varepsilon \leq \Re \nu$. Combining the two estimates directly yields the result. 
Lemma 7.4. Let $Y_{\nu}(x)$ be the $Y$-Bessel function with argument $x \in \mathbb{R}_{>0}$ and index $\nu \in \mathbb{C}$. Furthermore consider any small $\delta_{1}, \delta_{2}>0$, then for $|\Re \nu| \leq \delta_{1}$ we have

$$
Y_{\nu}(x) \ll e^{\pi|\Im \nu| / 2}\left(1+|\nu|^{1+2 \varepsilon}\right) \cdot \min \left\{\frac{1}{x^{\varepsilon}}, \frac{1}{\sqrt{x}}\right\},
$$

where $\varepsilon:=\delta_{1}+\delta_{2}$.

Proof. From [11, Proposition 9] we have for the $Y$-Bessel function with $|\Re \nu| \leq \delta_{1}$ the estimate

$$
e^{-\pi|\Im \nu| / 2} Y_{\nu}(x) \ll \begin{cases}(1+|\Im \nu|)^{\varepsilon} x^{-\varepsilon}, & \text { for } 0<x \leq 1+|\Im \nu| \\ (1+|\Im \nu|)^{-\delta_{2}} x^{\delta_{2}}, & \text { for } 1+|\Im \nu|<x \leq 1+|\nu|^{2} \\ x^{-1 / 2}, & \text { for } 1+|\nu|^{2}<x .\end{cases}
$$

Now for $x>1+|\nu|^{2}$ the bound claimed in the lemma dominates the estimate just quoted. In the interval $1+|\Im \nu|<x \leq 1+|\nu|^{2}$ we observe that

$$
1 \ll \frac{1+|\nu|}{\sqrt{x}},
$$

from which we conclude immediately with the appropriate bound form 7.5 that

$$
e^{-\pi|\Im \nu| / 2} Y_{\nu}(x) \ll\left(\frac{x}{1+|\Im \nu|}\right)^{\delta_{2}} \ll \frac{1+|\nu|^{1+2 \delta_{2}}}{\sqrt{x}} .
$$

Finally, for the interval $1 \leq x \leq 1+|\Im \nu|$ we observe that

$$
1 \ll \frac{1+|\Im \nu|^{1 / 2}}{\sqrt{x}},
$$

which upon again combining with the appropriate bound form 7.5 , yields

$$
e^{-\pi|\Im \nu| / 2} Y_{\nu}(x) \ll\left(\frac{1+|\Im \nu|}{x}\right)^{\varepsilon} \ll \frac{1+|\Im \nu|^{1 / 2+\varepsilon}}{\sqrt{x}} .
$$

Summarising the bounds for $0<x \leq 1$ and $x>1+|\nu|^{2}$, both from (7.5), with the bounds 7.6 and (7.7) for the two intervals as described in between those ranges, we quickly arrive at the statement of the lemma.

\subsubsection{The integral transform $\mathscr{J}$ of the $J$-Bessel function}

In the context of this thesis we also have to know a few properties of a certian integral transform of the $J$-Bessel function. The transform in question is defined by

$$
\mathscr{J}(y):=2 \pi \mathrm{i} \int_{\mathbb{R}} \frac{J_{2 \mathrm{i} w}(y)}{\sinh (\pi w)} h(w) d_{\mathrm{spec}} w,
$$

as it appears in the Kuznetsov formula in 2.7 . We analyse this in the following for $y \in \mathbb{R}_{>0}$ and Schwartz functions $h$, satisfying the conditions specified in Section 2.4

Lemma 7.5. We define the function $\psi: \mathbb{R}_{>0} \rightarrow \mathbb{R}_{>0}$ to be the inversion $\psi(x):=x^{-1}$ and consider the integral transform $\mathscr{J}$ as defined in (7.8), for functions $h$ as in Section 2.4 Then we find that

$$
\mathscr{J} \circ \psi, \mathscr{J} \in L^{1}\left(\mathbb{R}_{\geq 0}, \frac{d x}{x}\right) .
$$


Proof. In order to prove the lemma we first want to show that the limit

$$
\lim _{A \rightarrow \infty} \int_{1 / A}^{A}\left|\mathscr{J}\left(\frac{1}{x}\right)\right| \frac{d x}{x}
$$

exists. It suffices to establish a positive upper bound for the latter as $A \rightarrow \infty$. We do this by splitting the integral therein into

$$
\mathrm{I}_{1}(A):=\int_{1 / A}^{1}\left|\mathscr{J}\left(\frac{1}{x}\right)\right| \frac{d x}{x} \quad \text { and } \quad \mathrm{I}_{2}(A):=\int_{1}^{A}\left|\mathscr{J}\left(\frac{1}{x}\right)\right| \frac{d x}{x}
$$

and proceed by investigating their properties for growing $A$ separately. We are going start with $\mathrm{I}_{2}(A)$. After shifting the contour in $\mathscr{J}$ to $\Im(w)=-1 / 16$, we estimate the Bessel function with Lemma 7.1 to obtain

$$
\mathrm{I}_{2}(A) \ll \int_{1}^{A} \frac{d x}{x^{9 / 8}} \cdot \int_{\mathbb{R}} \frac{|w h(w-\mathrm{i} / 16)|}{1+|w|^{1 / 8}} d w \ll 1-\frac{1}{A^{1 / 8}},
$$

where we observe the lower bound $\cosh (\pi(w-\mathrm{i} / 16)) \gg \exp (\pi|w|)$ and the decay properties of $h$. The implied constant in 7.9 depends only on $h$. In order to bound $\mathrm{I}_{1}(A)$ we shift the contour in $\mathscr{J}$ to $\Im(w)=$ $1 / 16$ and as above bound the Bessel function employing Lemma 7.1. Hence we find that

$$
\mathrm{I}_{1}(A) \ll \int_{1 / A}^{1} \frac{d x}{x^{7 / 8}} \cdot \int_{\mathbb{R}}|h(w+\mathrm{i} / 16)|\left(|w|+|w|^{1 / 8}\right) d w \ll 1-\frac{1}{A^{1 / 8}},
$$

where the implied constant is again only influenced by the choice of $h$. Once more we have used that $\cosh (\pi(w-\mathrm{i} / 16)) \gg \exp (\pi|w|)$ and that $h$ is a Schwartz function. Combining (7.9) and 7.10) we immediately observe that

$$
\lim _{A \rightarrow \infty} \int_{1 / A}^{A}\left|\mathscr{J}\left(\frac{1}{x}\right)\right| \frac{d x}{x} \ll \lim _{A \rightarrow \infty}\left(1-\frac{1}{A^{1 / 8}}\right) \ll 1,
$$

with the implied constant only depending on the function $h$. The latter estimate (7.11) tells us that $\mathscr{J} \circ \psi \in$ $L^{1}\left(\mathbb{R}_{\geq 0}, \frac{d x}{x}\right)$. However performing the change of variables $x \mapsto x^{-1}$ in the integral

$$
\int_{0}^{\infty}|\mathscr{J}(x)| \frac{d x}{x}
$$

we see immediately that in fact we have $(\mathscr{J} \circ \psi)(x)=\mathscr{J}(x)$, which of course also gives us that $\mathscr{J} \in$ $L^{1}\left(\mathbb{R}_{\geq 0}, \frac{d x}{x}\right)$ and this finishes the proof of the lemma.

An integral role in our considerations is played by the following result. It provides a concrete evaluation of an integral transform in which we integrate the transform $\mathscr{J}$ from $(7.8)$ against a trigonometric function. Lemma 7.6 is in the very same style as the the formula [15, A.9] and we follow a very similar ansatz as used in [15] to prove our result.

Lemma 7.6. We define the operator $\mathrm{P}(g):=2 \Re(g)$, for functions $g: \mathbb{C} \rightarrow \mathbb{C}$ and let $h$ be a Schwartz function satisfying the properties as presented in Section 2.4. The integral transform $\mathscr{J}$ be as defined in 7.8). Now for any $A, B \in \mathbb{R}_{>0}$ and $C \in \mathbb{R}$ we define

$$
\mathscr{I}(A, B, C):=\mathrm{P} \int_{0}^{\infty} e\left((A+B) x+\frac{C}{x}\right) \mathscr{J}(4 \pi x \sqrt{A B}) \frac{d x}{x} .
$$


Then we have that

$$
\mathscr{I}(A, B, 0)=\frac{2}{\pi} \int_{\mathbb{R}} h(x)\left(\frac{\sqrt{A B}}{\max \{A, B\}}\right)^{2 \mathrm{i} x} d x .
$$

Furthermore for $C>0$ we get that

$$
\mathscr{I}(A, B, C)=-4 \pi^{2} \mathrm{i} \int_{\mathbb{R}} \frac{h(x)}{\sinh ^{2}(\pi x)} J_{2 \mathrm{i} x}(4 \pi \sqrt{A C}) \Im\left(J_{2 \mathrm{i} x}(4 \pi \sqrt{B C})\right) d_{\text {spec }} x
$$

and for $C<0$ that

$$
\mathscr{I}(A, B, C)=8 \pi \mathrm{i} \int_{\mathbb{R}} \frac{h(x)}{\tanh (\pi x)} Y_{2 \mathrm{i} x}(4 \pi \sqrt{|C| \max \{A, B\}}) K_{2 \mathrm{i} x}(4 \pi \sqrt{|C| \min \{A, B\}}) d_{\mathrm{spec}} x .
$$

Here J, $Y$ and $K$ denote the usual corresponding Bessel functions.

Proof. We begin the proof with a simple observation. Since the even function $h$ is real valued for real argument it is easy to see that $\left.\mathscr{J}\right|_{\mathbb{R}} \in \mathbb{R}$, too. Thus we see that

$$
\mathscr{I}(A, B, C)=2 \int_{0}^{\infty} \cos \left(2 \pi\left((A+B) x+\frac{C}{x}\right)\right) \mathscr{J}(4 \pi x \sqrt{A B}) \frac{d x}{x},
$$

which, after inserting the definition of $\mathscr{J}$, becomes

$$
\frac{4 \mathrm{i}}{\pi} \int_{\mathbb{R}} \frac{t h(t)}{\cosh (\pi t)} \int_{0}^{\infty} \cos \left(2 \pi\left((A+B) x+\frac{C}{x}\right)\right) J_{2 \mathrm{i} t}(4 \pi x \sqrt{A B}) \frac{d x}{x} d t
$$

where we interchange the order of integration of the $t$ and the $x$ integral. Swapping the integrals is permitted here due to Fubini's Theorem: After moving the contour in the $t$-integration to $\Im(t)=-\varepsilon / 2$ and employing Corollary 7.3 it is easily observed that the double integral in 7.16 converges in fact absolutely (cp. the decay properties of the function $h$ as described in Section 2.4). From the latter expression we can now quickly deduce the claimed formulae for the different values of $C$ under consideration. We start with the case of $C>0$ and shift the contour in the $t$-integration in $\sqrt{7.16}$ to $\Im(t)=-\varepsilon / 2$. Then with [17, Chapter $1 ; 1.6,6.39$, p.56 $]^{(\mathrm{I})}[\mathrm{II})$ we evaluate the inner integral in $(7.16)$, which therefore equals

$$
\int_{\mathbb{R}} \frac{-4 \mathrm{i} h(t) t}{\cosh (\pi t)} J_{2 \mathrm{i} t}(4 \pi \sqrt{A C})\left(J_{2 \mathrm{i} t}(4 \pi \sqrt{B C}) \sin (\pi \mathrm{i} t)+Y_{2 \mathrm{i} t}(4 \pi \sqrt{B C}) \cos (\pi \mathrm{i} t)\right) d t,
$$

\footnotetext{
${ }^{(\mathrm{I})}$ Here the function $f$ should read $f(x)=x^{-3 / 2} \cos \left(a x+b x^{-1}\right)$.

(II) Adopting the notation from the source the formula from [17] Chapter 1; 1.6, 6.39, p.56] which we want to employ is only valid in the case of $y<a$. However for our purposes we also need the formula to hold in the case of $y=a$, too. Thus we will give a short argument showing that this is indeed the case. In our situation we have for the index of the $J$-Bessel function in fact $\Re \nu=\delta>0$. Now we choose $a=y+\varepsilon$ and since this always exceeds $y$, for any $\varepsilon>0$, we know from [17] Chapter $1 ; 1.6,6.39$, p.56] that$$
\int_{0}^{\infty} \cos ((y+\varepsilon) x+b / x) J_{\nu}(x y) d x / x=-\pi J_{\nu}(c(\varepsilon))\left(J_{\nu}(d(\varepsilon)) \sin (\pi \nu / 2)+Y_{\nu}(d(\varepsilon)) \cos (\pi \nu / 2)\right),
$$

where

$$
c(\varepsilon):=b^{1 / 2}\left((2 y+\varepsilon)^{1 / 2}+\varepsilon^{1 / 2}\right) \text { and } d(\varepsilon):=b^{1 / 2}\left((2 y+\varepsilon)^{1 / 2}-\varepsilon^{1 / 2}\right) .
$$

Now by the Dominated Convergence Theorem and Corollary 7.3 we find that

$$
\lim _{\varepsilon \rightarrow 0} \int_{0}^{\infty} \cos ((y+\varepsilon) x+b / x) J_{\nu}(x y) d x / x=\int_{0}^{\infty} \cos (y x+b / x) J_{\nu}(x y) d x / x
$$

and by continuity that

$$
\lim _{\varepsilon \rightarrow 0} J_{\nu}(c(\varepsilon))\left(J_{\nu}(d(\varepsilon)) \sin (\pi \nu / 2)+Y_{\nu}(d(\varepsilon)) \cos (\pi \nu / 2)\right)=J_{\nu}(c(0))\left(J_{\nu}(d(0)) \sin (\pi \nu / 2)+Y_{\nu}(d(0)) \cos (\pi \nu / 2)\right) .
$$
}

Hence we conclude immediately that formula (7.17) also has to hold for $\varepsilon=0$, which in turn tells us that 17 Chapter 1 ; 1.6, 6.39, p.56] in fact is also true in the case of $y=a$. We recall that we have obtained this, using $\Re \nu=\delta>0$, which is essential for Corollary 7.3 to produce useful bounds to enable the application of the Dominated Convergence Theorem. 
where, after substituting for the $x$ integral, we moved the path of integration in the latter in the remaining $t$ integral back to $\Im(t)=\varepsilon / 2$. Shifting the contour is important here, since it allows us to work with a Bessel function in 7.16 with an index that has a small positive real part. This is necessary for the transformation we just executed via [17] to be valid in the case of $A=B$ (cp. the second footnote in this argument). We conclude (7.14) after rewrite the $Y$-Bessel function in the last integral in terms of $J$-Bessel functions. This is easily achieved by employing that for $\nu \in \mathbb{C} \backslash \mathbb{Z}$ and $x \in \mathbb{R}_{>0}$ from [10, 8.403.1] we have

$$
Y_{\nu}(x)=\frac{J_{\nu}(x) \cos (\pi \nu)-J_{-\nu}(x)}{\sin (\pi \nu)} .
$$

We proceed to values $C=-|C|<0$. Similarly as before we evaluate the inner integral in (7.16), this time by means of [17, Chapter $1 ; 1.6,6.38, \mathrm{p} .56]^{(\mathrm{III})}$ from which we quickly conclude that 7.16 now equals

$$
\frac{8 \mathrm{i}}{\pi} \int_{\mathbb{R}} \frac{t h(t)}{\cosh (\pi t)} \cos (\pi \mathrm{i} t) Y_{2 \mathrm{i} t}(4 \pi \sqrt{|C| \max \{A, B\}}) K_{2 \mathrm{i} t}(4 \pi \sqrt{|C| \min \{A, B\}}) d t,
$$

which immediately leads us to 7.15 ). We are left with the case of $C=0$. We shift the contour in $\mathscr{J}$ to $\Im(t)=-\varepsilon / 2$, noticing that the integrand is holomorphic here, and such that we avoid all the poles of the spectral measure $d_{\text {spec }} t$. As a result similarly to 7.16 we find that $\mathscr{I}(A, B, 0)$ equals

$$
\frac{4 \mathrm{i}}{\pi} \int_{\mathbb{R}} \frac{(t-\varepsilon \mathrm{i} / 2) h(t-\varepsilon \mathrm{i} / 2)}{\cosh (\pi(t-\varepsilon \mathrm{i} / 2))} \int_{0}^{\infty} \cos (2 \pi x(A+B)) J_{2 \mathrm{i} t+\varepsilon}(4 \pi x \sqrt{A B}) \frac{d x}{x} d t .
$$

We changed the contour here in order to be able to evaluate the inner Integral further. This can be done by means of [9, Chapter 8; 8.7: (27), p.36], employing that $A+B \geq 2 \sqrt{A B}$. We observe the equality $\cosh (\pi(t-\varepsilon \mathrm{i} / 2))=\cos (\pi(\mathrm{i} t+\varepsilon / 2))$ and find after some elementary manipulations that 7.19 becomes

$$
\frac{2}{\pi} \int_{\mathbb{R}} h(t-\varepsilon \mathrm{i} / 2)\left(\frac{\sqrt{A B}}{\max \{A, B\}}\right)^{2 \mathrm{i} t+\varepsilon} d t
$$

Since the integrand of the above is holomorphic at least in a small strip, we may shift the contour again to $\Im(t)=\varepsilon / 2$ and quickly arrive at 7.13 .

\subsubsection{Isolating the oscillation of the $J$ and $Y$-Bessel function}

Lemma 7.7. Let $J_{\nu}(x)$ and $Y_{\nu}(x)$ denote the usual Bessel functions for $x \in \mathbb{R}_{>0}$ and $\nu \in \mathbb{C}$ with $|\Re \nu|<$ 1/2. Then we have

$$
J_{\nu}(2 \pi x)=\frac{1}{2 \pi \sqrt{x}}\left(e(x) \Omega_{+}(\nu, x)+e(-x) \Omega_{-}(\nu, x)\right)
$$

and

$$
Y_{\nu}(2 \pi x)=\frac{-\mathrm{i}}{2 \pi \sqrt{x}}\left(e(x) \Omega_{+}(\nu, x)-e(-x) \Omega_{-}(\nu, x)\right),
$$

\footnotetext{
${ }^{\text {(III) }}$ As in the case of the formula [17. Chapter $1 ; 1.6,6.39$, p.56] the present one $([17$. Chapter $1 ; 1.6,6.38$, p.56]) is also valid in the case of $y=a$, adopting again the notation of the source. This can be seen by a very similar argument as given for the case of [17. Chapter $1 ; 1.6,6.39$, p.56] (cp. the second footnote in this argument). Just as in the case of $C>0$, we have to perform similar shifts of the contour here in 7.16 before applying [17], for the transformation to be valid in the case of $A=B$, and then afterwards moving the contour back to obtain the desired formula in the form in 7.18 .
} 
where

$$
\Omega_{ \pm}(\nu, x):=\frac{e^{\mp \mathrm{i}(\nu \pi / 2+\pi / 4)}}{\Gamma(\nu+1 / 2)} \int_{0}^{\infty} e^{-y}\left(y \pm \frac{\mathrm{i} y^{2}}{4 \pi x}\right)^{\nu-1 / 2} d y
$$

For the latter function and its derivatives we have the bounds

$$
\frac{\partial^{j}}{\partial x^{j}} \Omega_{ \pm}(\nu, x) \ll_{j} \frac{\left(1+|\Im \nu|^{j+1 / 2}\right) e^{\pi|\Im \nu|}}{x^{j}}
$$

for any $j \geq 0$.

Proof. From [18, p.168] with $\beta=0$ we have

$$
H_{\nu}^{(1)}(x)=\left(\frac{2}{\pi x}\right)^{1 / 2} \frac{e^{\mathrm{i}(x-\nu \pi / 2-\pi / 4)}}{\Gamma(\nu+1 / 2)} \int_{0}^{\infty} e^{-y}\left(y+\frac{\mathrm{i} y^{2}}{2 x}\right)^{\nu-1 / 2} d y
$$

and

$$
H_{\nu}^{(2)}(x)=\left(\frac{2}{\pi x}\right)^{1 / 2} \frac{e^{-\mathrm{i}(x-\nu \pi / 2-\pi / 4)}}{\Gamma(\nu+1 / 2)} \int_{0}^{\infty} e^{-y}\left(y-\frac{\mathrm{i} y^{2}}{2 x}\right)^{\nu-1 / 2} d y
$$

in both cases for any $\nu \in \mathbb{C}$ with $\Re \nu>-1 / 2$ and $x>0$. Combining both these formulae in [10, 8.481 \& 8.482 ], i.e respectively

$$
J_{\nu}(x)=\frac{1}{2}\left(H_{\nu}^{(1)}(x)+H_{\nu}^{(2)}(x)\right) \quad \text { and } \quad Y_{\nu}(x)=\frac{\mathrm{i}}{2}\left(H_{\nu}^{(2)}(x)-H_{\nu}^{(1)}(x)\right),
$$

we obtain immediately 7.20 and 7.21 , as desired. It remains to establish the bounds for $\Omega_{ \pm}(\nu, x)$ and its derivatives in (7.23). To this end we use Stirling approximation to see that

$$
\Gamma^{-1}(\nu+1 / 2) \ll e^{\pi|\Im \nu| / 2}(1+|\Im \nu|)^{-\Re \nu} \ll e^{\pi|\Im \nu| / 2}\left(1+|\Im \nu|^{1 / 2}\right) .
$$

Furthermore for $\Re \nu<1 / 2$ we have

$$
e^{ \pm \pi \Im \nu / 2}\left(y \pm \frac{\mathrm{i} y^{2}}{4 \pi x}\right)^{\nu-1 / 2} \ll \frac{e^{\pi|\Im \nu| / 2}}{y^{1 / 2-\Re \nu}}
$$

Now differentiating with respect to $x$ in (7.22) under the integral $j$-times and employing (7.24) we obtain

$$
e^{ \pm \pi \Im \nu / 2} \frac{\partial^{j}}{\partial x^{j}}\left(y \pm \frac{\mathrm{i} y^{2}}{4 \pi x}\right)^{\nu-1 / 2} \ll_{j} \frac{1}{x^{j}} \cdot \frac{e^{\pi|\Im \nu| / 2}\left(|\Im \nu|+|\Im \nu|^{j}\right)}{y^{1 / 2-\Re \nu}}
$$

Finally employing the bounds 7.24 and 7.25 together with the estimate for the gamma function we conclude 7.23 , where we combine to one bound for $\Omega_{ \pm}(\nu, x)$ and its derivatives.

Lemma 7.8. Let $\Omega_{ \pm}(\nu, x)$ be as in $(7.22$ with support on $[Y, Z]$, where $0<Y<1$ and $Z>$ e such that $1 \ll Y Z$. Furthermore let $G_{ \pm}$be the functions as defined in $(7.26$. Then for any $x \in[Y, Z]$ we have via the inverse Mellin transform

$$
\Omega_{ \pm}(\nu, x)=G_{ \pm}(x)=\frac{1}{2 \pi \mathrm{i}} \int_{(\sigma)} \widehat{G}_{ \pm}(s) x^{-s} d s,
$$

where for any $\Re s=\sigma \geq 0$ and $j \geq 2$ we have

$$
\int_{(\sigma)}\left|\widehat{G}_{ \pm}(s)\right||d s| \ll\left(1+|\Im \nu|^{j+1 / 2}\right) e^{\pi|\Im \nu|}\left(Z^{\sigma}+\log Z\right) .
$$


Proof. Let $g: \mathbb{R} \rightarrow \mathbb{R}_{\geq 0}$ be a smooth function with support on $[Y / 2,3 Z / 2]$, which equals one on $[Y, Z]$ and satisfies $g^{(j)}(x) \ll_{j} Y^{-j}$ on $[Y / 2, Y]$ and $g^{(j)}(x) \ll_{j} Z^{-j}$ on $[Z, 3 Z / 2]$, both for $j \geq 0$. We define functions $G_{ \pm}: \mathbb{R} \rightarrow \mathbb{C}$ via

$$
G_{ \pm}(x):=g(x) \Omega_{ \pm}(\nu, x)
$$

and highlight the fact that clearly $\left.\Omega_{ \pm}(\nu, \cdot)\right|_{[Y, Z]}=\left.G_{ \pm}\right|_{[Y, Z]}$. Now we want to estimate the Mellin transform

$$
\widehat{G}_{ \pm}(s)=\int_{0}^{\infty} G_{ \pm}(x) x^{s-1} d x
$$

of the function just introduced. Bounding the latter integral directly, quickly leads to the bound

$$
\widehat{G}_{ \pm}(s) \ll\left(1+|\Im \nu|^{1 / 2}\right) e^{\pi|\Im \nu|}\left(Z^{\sigma}+\log Z\right),
$$

for any $\sigma \geq 0$. Here we employed $(7.23)$ to control the function $\Omega_{ \pm}$and the condition $1 \ll Y Z$ to obtain the presented bound in the case of $\sigma=0$. On the other hand by repeated integration by parts of the integral 7.27, we see that the Mellin transform equals

$$
\frac{(-1)^{j}}{s(s+1) \ldots(s+j-1)} \sum_{k=0}^{j}\left(\begin{array}{l}
j \\
k
\end{array}\right) \int_{Y / 2}^{3 Z / 2} g^{(j-k)}(x) \Omega_{ \pm}^{(k)}(\nu, x) x^{s+j-1} d x
$$

where we used the Leibniz rule to differentiate the function $G_{ \pm}$multiple times. Bounding this expression for the Mellin transform requires us to treat several intervals within the range of integration separately, since the function $g$ and its derivatives have different growth properties therein. Again we use (7.23) to bound $\Omega_{ \pm}$and its derivatives. On $[Y / 2, Y]$ we invoke $1 \ll Y Z$ and find

$$
\begin{aligned}
\sum_{k=0}^{j}\left(\begin{array}{l}
j \\
k
\end{array}\right) \int_{Y / 2}^{Y} & \ldots d x \\
& \ll e^{\pi|\Im \nu|} \sum_{k=0}^{j} Y^{k-j}\left(1+|\Im \nu|^{k+1 / 2}\right) \int_{Y / 2}^{Y} x^{\sigma-1+j-k} d x \ll\left(1+|\Im \nu|^{j+1 / 2}\right) e^{\pi|\Im \nu|} \log Z
\end{aligned}
$$

where the last bound summarises the estimates one obtains considering both $\sigma=0$ and $\sigma>0$ separately. In a similar fashion we then also find that

$$
\sum_{k=0}^{j}\left(\begin{array}{l}
j \\
k
\end{array}\right) \int_{Z}^{3 Z / 2} \ldots d x \ll\left(1+|\Im \nu|^{j+1 / 2}\right) e^{\pi|\Im \nu|} Z^{\sigma}
$$

and

$$
\sum_{k=0}^{j}\left(\begin{array}{l}
j \\
k
\end{array}\right) \int_{Y}^{Z} \ldots d x \ll\left(1+|\Im \nu|^{j+1 / 2}\right) e^{\pi|\Im \nu|}\left(Z^{\sigma}+\log Z\right),
$$

with the condition $1 \ll Y Z$ again proving essential in order to derive the estimate in the case of $\sigma=0$. Combining the latter three estimates in (7.29) and furthermore summarising the resulting bound with (7.28) yields

$$
\widehat{G}_{ \pm}(s) \ll \frac{\left(1+|\Im \nu|^{j+1 / 2}\right) e^{\pi|\Im \nu|}}{1+|s|^{j}}\left(Z^{\sigma}+\log Z\right), \text { for any } \sigma \geq 0,
$$

from which, upon choosing $j \geq 2$, we immediately conclude the lemma. 


\subsection{The $K$-Bessel function}

\subsubsection{The inverse Mellin transform of the $K$-Bessel function}

We denote by $K_{\nu}(x)$ be the $K$-Bessel function for index $\nu \in \mathbb{C}$ with $\Re \nu \geq 0$ and of argument $x \in \mathbb{R}_{>0}$. A classical tool is to write this Bessel function via its inverse Mellin transform as

$$
K_{\nu}(x)=\frac{1}{2 \pi \mathrm{i}} \int_{(\sigma)} 2^{s-2} \Gamma\left(\frac{s-\nu}{2}\right) \Gamma\left(\frac{s+\nu}{2}\right) x^{-s} d s,
$$

for any $\Re s=\sigma>\Re \nu \geq 0$, a formula which is widely known. The latter integral representation follows at once by plugging the Mellin transform of the $K$-Bessel function, for which for instance from [8, 6.8 (26) / p.331] we know that it is given by

$$
\int_{0}^{\infty} K_{\nu}(x) x^{s-1} d x=2^{s-2} \Gamma\left(\frac{s-\nu}{2}\right) \Gamma\left(\frac{s+\nu}{2}\right),
$$

for all $\Re s>\Re \nu$, into the inverse Mellin transform formula. The following result confirms, also providing a concrete bound, absolute convergence of the integral in 7.31.

Lemma 7.9. Let $K_{\nu}(x)$ be the $K$-Bessel function for $\nu \in \mathbb{C}$ with $\Re \nu \geq 0$ and of argument $x \in \mathbb{R}_{>0}$. Writing the $K$-Bessel function as its own inverse Mellin transform as in (7.31), we find that for any $\Re s=$ $\sigma>\Re \nu \geq 0$ the integral in the latter transform admits

$$
\int_{(\sigma)}\left|2^{s-2} \Gamma\left(\frac{s-\nu}{2}\right) \Gamma\left(\frac{s+\nu}{2}\right)\right||d s| \ll e^{-\pi|\Im \nu| / 4}\left(1+|\Im \nu|^{\max \{3(\sigma-1) / 2, \sigma\}}\right) .
$$

Proof. We assume in the following that $\sigma>\Re \nu \geq 0$ and recall the $K$-Bessel function in the integral representation as given in 7.31 . The task is now to prove absolute convergence of the integral therein, i.e. the inverse Mellin transform of $K_{\nu}(x)$. To this end Stirling approximation provides the bound

$$
\Gamma\left(\frac{s-\nu}{2}\right) \Gamma\left(\frac{s+\nu}{2}\right) \ll \frac{\left(1+\left|\Im\left(\frac{s-\nu}{2}\right)\right|\right)^{\Re\left(\frac{s-\nu}{2}\right)-\frac{1}{2}}\left(1+\left|\Im\left(\frac{s+\nu}{2}\right)\right|\right)^{\Re\left(\frac{s+\nu}{2}\right)-\frac{1}{2}}}{e^{\frac{\pi}{2}\left\{\left|\Im\left(\frac{s-\nu}{2}\right)\right|+\left|\Im\left(\frac{s+\nu}{2}\right)\right|\right\}}} .
$$

We deal with the exponential factor in the latter estimate first. Carefully examining all possible combinations of $\Im s$ and $\Im \nu$, being less than, bigger than or equal to zero, individually leads to

$$
|\Im(s / 2-\nu / 2)|+|\Im(s / 2+\nu / 2)|=\max \{|\Im s|,|\Im \nu|\} \geq|\Im s| / 2+|\Im \nu| / 2 .
$$

Thus the exponential contribution in the bound for the gamma functions may be estimated by

$$
\exp (-\pi(|\Im s|+|\Im \nu|) / 4)
$$

For the polynomial part of 7.32 we obtain the estimates

$$
\begin{cases}1+|\Im s|^{3(\sigma-1) / 2}+|\Im \nu|^{3(\sigma-1) / 2}, & \text { for } \sigma>1+\Re \nu, \\ 1+|\Im s|^{\max \{0,(\sigma-1+\Re \nu) / 2\}}+|\Im \nu|^{\max \{0,(\sigma-1+\Re \nu) / 2\}}, & \text { for } \sigma \leq 1+\Re \nu\end{cases}
$$

Summarising the bounds obtained above, from 7.32 we find

$$
\Gamma\left(\frac{s-\nu}{2}\right) \Gamma\left(\frac{s+\nu}{2}\right) \ll \frac{1+|\Im s|^{\max \{3(\sigma-1) / 2, \sigma\}}+|\Im \nu|^{\max \{3(\sigma-1) / 2, \sigma\}}}{e^{\pi(|\Im s|+|\Im \nu|) / 4}},
$$

from which we immediately deduce the lemma. 


\subsubsection{The Whittaker and the $K$-Bessel function}

Lemma 7.10. Let $w \in \mathbb{R}$ and $z \in \mathbb{C} \backslash(-\infty, 0]$, then the $K$-Bessel function satisfies

$$
K_{\mathrm{i} w}(z)=\left(\frac{\pi}{2 z}\right)^{1 / 2} W_{0, \mathrm{i} w}(2 z),
$$

where the Whittaker function is defined by

$$
W_{0, \mathrm{i} w}(2 z):=\frac{e^{-z}}{\Gamma(1 / 2+\mathrm{i} w)} \int_{0}^{\infty} e^{-y}\left(y+\frac{y^{2}}{2 z}\right)^{\mathrm{i} w-1 / 2} d y
$$

Proof. We can write every $z \in \mathbb{C} \backslash(-\infty, 0]$ as $z=x u$, where $x>0$ and $|\arg (u)|<\pi, u \neq 0$. Then for $w \in \mathbb{R}$ from [10,9.222.2] we obtain with $\lambda=0$ and $\mu=\mathrm{i} w$ for the Whittaker function

$$
W_{0, \mathrm{i} w}(2 x u)=\frac{e^{-x u}}{\Gamma(1 / 2+\mathrm{i} w)} \int_{0}^{\infty} e^{-y}\left(y+\frac{y^{2}}{2 x u}\right)^{\mathrm{i} w-1 / 2} d y .
$$

With the change of variables $y \mapsto x y$ in the above integral we see that the Whittaker function equals

$$
\frac{e^{-x u} x^{1 / 2+\mathrm{i} w}}{\Gamma(1 / 2+\mathrm{i} w)} \int_{0}^{\infty} e^{-x y}\left(y+\frac{y^{2}}{2 u}\right)^{\mathrm{i} w-1 / 2} d y
$$

However by considering the integral representation of the $K$-Bessel function as given in [10, 8.432.8] we find that 7.33 in fact equals

$$
K_{\mathrm{i} w}(x u)\left(\frac{2 x u}{\pi}\right)^{1 / 2}
$$

which gives us the statement of the lemma. 


\section{Bibliography}

[1] Blomer, V., Khan, R., Young, M.: Distribution of mass of holomorphic cusp forms. Duke Mathematical Journal. Vol. 162, No. 14 (2013)

[2] Blomer, V., Milićević, D.: Kloosterman sums in residue classes. Journal of the European Mathematical Society (JEMS). 17, No. 1, 51-69 (2015)

[3] Brüdern, J.: Einführung in die analytische Zahlentheorie. Springer-Verlag Berlin Heidelberg GmbH (1995)

[4] Bruggeman, R.W.: Fourier Coefficients of Cusp Forms. Inventiones mathematicae. 45, 1-18 (1978)

[5] Cohen, H., Oesterlé, J.: Dimension des espaces de formes modulaires. Modular Functions of One Variable VI (Proceedings International Conference, University of Bonn, Sonderforschungsbereich Theoretische Mathematik, July 2-14, 1976, Edited by J.-P. Serre and D.B. Zagier), p.69-78. Lecture Notes in Mathematics. Volume 627. Springer-Verlag Berlin Heidelberg New York (1977)

[6] Deshouillers, J.-M., Iwaniec, H.:Kloosterman Sums and Fourier Coefficients of Cusp Forms. Inventiones mathematicae. 70, 219-288 (1982)

[7] Duke, W., Friedlander, J.B., Iwaniec, H.: The subconvexity problem for Artin L-functions. Inventiones mathematicae. 149, 489-577 (2002)

[8] Erdélyi, A., Magnus, W., Oberhettinger, F., Tricomi, F. G.: Tables of Integral Transforms. Volume 1. McGraw-Hill Book Company, Inc. (1954)

[9] Erdélyi, A., Magnus, W., Oberhettinger, F., Tricomi, F. G.: Tables of Integral Transforms. Volume 2. McGraw-Hill Book Company, Inc. (1954)

[10] Gradshteyn, I. S. and Ryzhik, I. M.: Table of Integrals, Series, and Products. Sixth Edition. Academic Press (2000)

[11] Harcos, G., Michel, P.: The subconvexity problem for Rankin-Selberg L-functions and equidistribution of Heegner points. II. Inventiones mathematicae 163, 581-655 (2006)

[12] Hejhal, D. A.: The Selberg Trace Formula for $P S L(2, \mathbb{R})$, Volume 2. Lecture Notes in Mathematics. Volume 1001. Springer-Verlag Berlin Heidelberg New York Tokyo (1983)

[13] Iwaniec, H.: Introduction to the Spectral Theory of Automorphic Forms. Revista Matemática Iberoamericana (1995)

[14] Iwaniec, H., Kowalski, E.: Analytic Number Theory. American Mathematical Society. Colloquium Publications. Volume 53 (2004) 
[15] Iwaniec, H., Li, X.: The orthogonality of Hecke eigenvalues. Compositio Math. 143, 541-565 (2007)

[16] Kuznecov, N.V. Petersson's Conjecture for Cusp Forms of weight zero and Linnik's Conjecture. Sums of Kloosterman Sums. Math. USSR Sb. 39, 299-342 (1981)

[17] Oberhettinger, F.: Tables of Bessel Transforms. Springer-Verlag Berlin Heidelberg New York (1972)

[18] Watson, G. N.: A Treatise on the theory of Bessel functions. Second Edition. Cambridge University Press (1966) 

